\title{
Embryological and surgical aspects of the tethered cord
}

Citation for published version (APA):

van Aalst, J. (2009). Embryological and surgical aspects of the tethered cord. [Doctoral Thesis, Maastricht University]. Datawyse / Universitaire Pers Maastricht. https://doi.org/10.26481/dis.20091218ja

Document status and date:

Published: 01/01/2009

DOI:

10.26481/dis.20091218ja

Document Version:

Publisher's PDF, also known as Version of record

\section{Please check the document version of this publication:}

- A submitted manuscript is the version of the article upon submission and before peer-review. There can be important differences between the submitted version and the official published version of record.

People interested in the research are advised to contact the author for the final version of the publication, or visit the DOI to the publisher's website.

- The final author version and the galley proof are versions of the publication after peer review.

- The final published version features the final layout of the paper including the volume, issue and page numbers.

Link to publication

\footnotetext{
General rights rights.

- You may freely distribute the URL identifying the publication in the public portal. please follow below link for the End User Agreement:

www.umlib.nl/taverne-license

Take down policy

If you believe that this document breaches copyright please contact us at:

repository@maastrichtuniversity.nl

providing details and we will investigate your claim.
}

Copyright and moral rights for the publications made accessible in the public portal are retained by the authors and/or other copyright owners and it is a condition of accessing publications that users recognise and abide by the legal requirements associated with these

- Users may download and print one copy of any publication from the public portal for the purpose of private study or research.

- You may not further distribute the material or use it for any profit-making activity or commercial gain

If the publication is distributed under the terms of Article $25 \mathrm{fa}$ of the Dutch Copyright Act, indicated by the "Taverne" license above, 
Embryological and Surgical Aspects of the Tethered Cord 
(C) 2009 J. van Aalst, Maastricht

Layout: Tiny Wouters

Production: Datawyse | Universitaire Pers Maastricht

ISBN: 9789052788845

Financial support for the printing of this thesis was kindly provided by: Baxter Biosurgery, B. Braun Medical B.V., Carl Zeiss B.V., Codman (Johnson \& Johnson), Medtronic Neuromodulation, Promedics Medical Systems B.V. and Synthes B.V.

The copyright of the articles that have been published has been transferred to the respective journals. 


\title{
Embryological and Surgical Aspects of the Tethered Cord
}

\author{
PROEFSCHRIFT
}

ter verkrijging van de graad van doctor aan de Universiteit Maastricht, op gezag van de Rector Magnificus, Prof. mr. G.P.M.F. Mols volgens het besluit van het College van Decanen in het openbaar te verdedigen op vrijdag 18 december 2009 om 12.00 uur

door

Jasper van Aalst

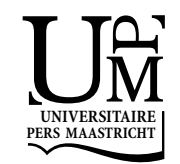




\section{Promotores}

Prof. dr. J.S.H. Vles

Prof. dr. E.A.M. Beuls (em)

\section{Beoordelingscommissie}

Prof. dr. J.J. van Overbeeke, voorzitter

Prof. dr. F. van Calenbergh (Universitair Ziekenhuis Leuven)

Dr. C.E.M. de Die-Smulders

Prof. dr. L.W. van Rhijn

Dr. D.A. Sival (Universitair Medisch Centrum Groningen) 


\section{Contents}

$\begin{array}{lll}\text { Chapter } 1 \text { Introduction } & 7\end{array}$

Chapter 2 In vitro high-field magnetic resonance imaging-documented anatomy of a fetal myelomeningocele at 20 weeks' gestation Journal of Neurosurgery: Spine 2003;98(2 Suppl): 210-214

Chapter 3 Anatomy and surgery of the infected dermal sinus of the lower spine

Childs Nervous system 2006; 22:1305-1315

Chapter 4 The spinal dermal sinus-like stalk

Childs Nervous system 2009;25:191-197

Chapter 5 Spinal congenital dermal sinus in a chick embryo model Laboratory investigation

Journal of Neurosurgery: Pediatrics 2009;3:24-28

Chapter 6 The intermediate type split cord malformation

Childs Nervous System 2005;21 1020-1024

Chapter 7 The Currarino triad: neurosurgical considerations

Neurosurgery 2006;58:924-929

Chapter 8 Intraspinal dermoid and epidermoid tumors: report of 18 cases and reappraisal of the literature

Pediatric Neurosurgery 2009;45:281-290

Chapter 9 General discussion and conclusion

Nederlandse samenvatting

Dankwoord

List of publications

Curriculum Vitae

Colour figures 



\section{Chapter 1}

Introduction 



\section{Spina bifida}

As early as in 1610, posthumous work of the Dutch clinician Pieter van Foreest (1522-1597) was published, in which he described the first surgically treated spina bifida patient. Three decades later, another Dutch physician, Nicolaes Tulp (1593-1674) introduced the term 'spina bifida' (from Özek et al. ${ }^{12}$ ).

Since then, numerous physicians, surgeons and researchers around the world have tried to understand this complex disease with its many appearances.

The term "spina bifida" refers to a malformation disturbing the dorsal midline axis of the spine and spinal cord. A highly variable expression in severity is seen, ranging from a mild defect, such as an absent spinous process, to a lifethreatening myelomeningocele.

All types of spina bifida are covered within the term "spinal dysraphism". Spina bifida aperta and spina bifida cystica are midline spinal malformations communicating with the external environment. Spina bifida occulta refers to skin-covered spina bifida lesions that may lead to neurological deterioration, usually by tethering of the spinal cord as the spine grows during childhood ${ }^{10}$.

Most patients do not only suffer from spinal cord lesions and open vertebral arches, but also display a wide variety of associated symptoms and even anatomical deformities in different parts of their bodies.

Nowadays, treatment of spina bifida patients has significantly improved because knowledge concerning the different aspects of the disease has increased. In many countries multi-disciplinary spina bifida clinics have been founded which has resulted in better treatment of these patients. However, new treatment options are still explored, and on many subjects consensus still has to be achieved. In 1997, the first human spina bifida patient underwent intrauterine surgical closure of a myelomeningocele defect. This therapy was started after promising results had been observed in animal studies. Despite these encouraging results in animals the effect on sensorimotor function after fetal surgery for spina bifida remains disappointing. However, the degree of hindbrain herniation significantly decreased in intrauterine treated patients, as well as the number of patients requiring ventriculoperitoneal shunting ${ }^{3,19,20}$. Although the incidence of severe spina bifida aperta is declining ${ }^{4}$ due to better prevention (i.e. folic acid) and improved prenatal imaging and counselling, new spina bifida patients are still frequently brought to our attention. Due to the decline in severe spina bifida aperta, an increasing part of the overall patient group presents with less severe neurological symptoms in combination with some type of spina bifida occulta ${ }^{4}$. In many cases these patients are able to walk and live rather normal lives.

Nevertheless, in patients with spina bifida occulta the spinal cord may be involved in the embryological malformation as well, and a congenital fixation of 
the cord at the level of the malformation might occur, giving rise to a tethered cord.

\section{The tethered cord}

The word "tether" is defined as a rope or chain by which an animal is fastened so that it can range only within a certain radius ${ }^{22}$.

A tethered cord is characterized by a spinal cord which is fixated at a certain level. Due to this fixation increased tension on the cord during normal daily activities is thought to result in a decrease of perfusion, after a certain period of time followed by ischemic changes within the spinal $\operatorname{cord}^{6,7,9,22}$, giving rise to neurological symptoms.

Many mechanisms giving rise to a tethering of the spinal cord have been described in literature. In most cases the cord is tethered at lumbar or lumbosacral level, but tethering may also occur at thoracic or cervical level, or even at the craniocervical junction in case of a high cervical spina bifida.

In most patients presenting with a spina bifida of the lower back a low-lying conus medullaris under the level of the L1-L2 interspace is observed on magnetic resonance imaging. This low-lying conus results from of a failure during embryological development preventing the physiologically occurring 'ascensus medullaris', due to the congenital fixation of the spinal cord.

The ascensus medullaris is explained by the fact that, during fetal life, the spinal cord grows much slower than the vertebral column. This leads to a progressive disparity between termination of the spinal cord and that of the spine, which results in a progressive ascent of the conus medullaris ${ }^{22}$.

At 20 weeks' gestation the conus medullaris of the spinal cord is situated at a level between the L4 and L5 vertebra. By 40 weeks' gestation the conus ascends to the level of $L 3$, and at the age of two months it reaches the adult L1-L2 level ${ }^{1}$.

With regard to tethered cord patients, two questions must be answered:

1. What are the mechanisms tethering the spinal cord?

2. How do the spinal cord or conus medullaris react to this tethering?

\section{What are the mechanisms tethering the spinal cord?}

Common causes are a tight filum terminale or tethering of the neural placode in a mass of scar tissue in case of a previously closed myelomeningocele.

The filum terminale can be described as a viscoelastic band attached to the conus medullaris which fixates and buffers the distal spinal cord during 
physiological movements of the spine. It develops during the process of secondary neurulation from an undifferentiated, pluripotent caudal cell mass called the caudal eminence and results from a regression of the caudalmost part of the neural tube 8 . This regression or "retrogressive differentiation" begins as early as day 43 to 48 and extends into the early postnatal period (from Yamada $^{22}$ ).

In patients with a myelomeningocele, the ascensus medullaris is prevented because the spinal cord is tethered at the level of the protrusion of the spinal cord and its meningeal covering.

After surgical closure of the myelomeningocele defect, the spinal cord remains situated at the level of the defect and is subsequently embedded in a mass of scar tissue. A secondary ascensus medullaris does not take place during the immediate postoperative period and this fixation of the spinal cord or conus medullaris results in a tethered cord.

Many other malformations associated with a tethered cord are known as well. A congenital spinal dermal sinus is a hollow tract, lined by stratified squamous epithelium. The most dorsal part of the tract presents as an open dimple in the midline skin of the neuraxis, with a distribution ranging from the tip of the nose to sacral level. The ventral part of the sinus leads through the underlying tissue layers and although a penetration variable in depth is reported, in most cases a connection with the central nervous system is found. This tract tethers the spinal cord by means of a direct tight connection between the skin and the spinal cord.

The first description found of a spinal dermal sinus is that of Dubreuilh $(1887)^{5}$ reporting on an intramedullary abscess due to a cervical dermal sinus with a congenital dermoid tumor, after which in the early $20^{\text {th }}$ century more cases were reported. In these first reports it was sometimes regarded as a complicated form of a pilonidal sinus ${ }^{15,17}$. However in 1926, Moise described a patient in whom a meningitis developed secondary to a sacral dermal sinus ${ }^{11}$. He suggested an etiology different from that of a pilonidal sinus. In 1934, Walker introduced the term 'congenital dermal sinus', which is still used in current literature ${ }^{21}$.

The mechanism by which a split cord malformation (SCM) tethers the spinal cord is not based on caudal fixation of the cord, but is merely due to the fixation of the cord at the level of the SCM. In case of a SCM the divided cord is tethered by fibrous bands or a rigid septum in between the two hemicords (dependent on whether a type I or type II SCM is present), thereby preventing the ascensus medullaris of the cord. Two types of a split cord malformation (SCM) are distinguished, based on the work of Pang et al. ${ }^{13,14}$. A type I SCM is described as two hemicords, each one being surrounded by its own dural sac and separated by a firm, osteocartilaginous septum, whereas in a type II SCM 
the two hemicords are lying within a single dural sac only being separated by a nonrigid fibrous septum ${ }^{14}$.

\section{How do the spinal cord or conus medullaris react to tethering?}

The pathophysiology of the tethered cord is not fully understood. In general, traction on the cord is thought to result in local hypoxemia and impaired metabolic function, followed by cord dysfunction, ultimately resulting in neurological deficit.

Several animal models have been used to study the effect of traction on the spinal cord with regard to regional spinal cord blood flow.

Independent of the mechanism giving rise to a tethering, this mechanism causes traction on the spinal cord. Kang et al. ${ }^{6}$ described an experimental animal model in feline kittens, in which the effect of tethering on regional spinal cord blood flow was studied. Tethering of the spinal cord induced a reduction of regional spinal cord blood flow in the distal spinal cord close to the tethering by $32 \%$ two weeks after tethering and by $67 \%$ ten weeks after tethering. Untethering of the cord resulted in an increase of regional blood flow, restoring it to the normal level. However, after eight weeks of tethering, the regional blood flow did not increase ${ }^{6}$.

Yamada et al. also used a feline model to study the effect of tethering on the spinal cord. In an experimental setting, traction to the filum terminale was applied by means of a measured weight. They concluded from their experiments that traction on the filum terminale has the greatest effect on the more caudal segments of the cord. The spinal cord segments cranial to the lowest dentate ligament attachments did not elongate. In addition, the lower segments stretched faster than the higher segments and remained slightly longer elongated after release of the traction. They also observed that the viscoelasticity of the filum terminale is much greater than that of any cord segments and therefore protects the spinal cord from overstretching ${ }^{18,23}$. According to Yamada et al. metabolic derangement and impaired blood flow seem to be associated with a tethered spinal cord $^{22}$.

In humans, blood flow in tethered cords was studied by Schneider et al. who performed a laser-Doppler flowmetry in ten children who underwent surgical untethering. They observed a significant increase in spinal cord blood flow after untethering $^{16}$. However, Brezner et al. performed ultrasonography of spinal cord pulsations in 106 children with a tethered cord and a myelomeningocele. They concluded from their observations that the absence of cord pulsation correlated with the presence of symptoms, but stated that in these myelomeningocele patients, these findings may have been caused by structural defects of the cord as well as by postoperative effects ${ }^{2}$. 


\section{The tethered cord syndrome}

A patient with a tethered cord may present with a tethered cord syndrome.

If a tethered cord is observed at radiological examination, this finding only describes the radiologically fixated position of the spinal cord. In many patients, cutaneous stigmata may be present as well, including haemangiomas, nevi, hypertrichosis, dimples, and subcutaneous lipomas or asymmetrical natal clefts $^{9}$. However, the diagnosis of a tethered cord syndrome is not based on radiological or dermatological abnormities but instead on neurological symptoms. These neurological symptoms may be subtle and a wide variety of signs and symptoms may be displayed by the patient presenting with a tethered cord syndrome. Common clinical symptoms are urological dysfunction, pain in the back or lower extremities, gait disorders, motor weakness, sensory disturbances, and orthopaedic foot and spine deformities ${ }^{4}$.

\section{Embryology of the neural tube}

All cases of spina bifida and tethered cord are more easily understood, when they can be compared to the normal embryological development of the central nervous system.

In the second week of embryological development, the embryo consists of two layers (epiblast and hypoblast), which are enclosed by two spheres; the amniotic cavity and yolk sac respectively. Both cystic spheres are surrounded by the extraembryonic mesoderm (Figure 1.1).

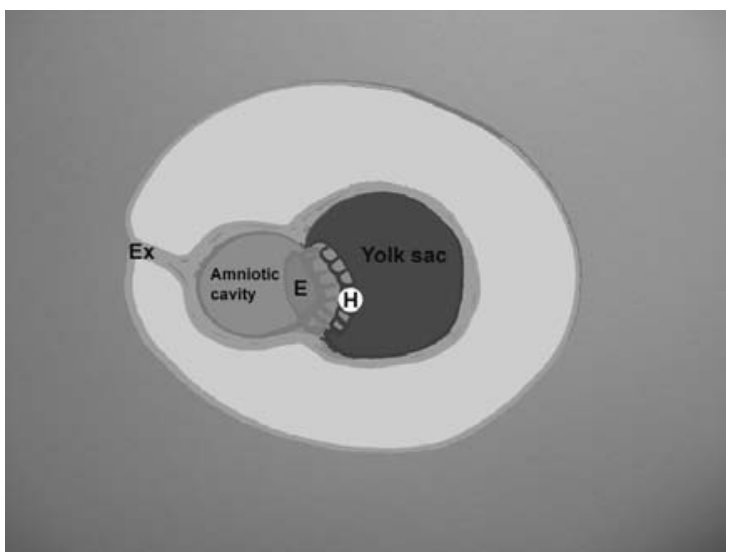

Figure 1.1 Ex = Extraembryonic mesoderm, $\mathrm{E}=$ Epiblast, $\mathrm{H}=$ Hypoblast.

See page 154 for colour figure. 
In the beginning of the third week (day 15-16), the primitive streak appears in the epiblast, from which cells start to proliferate and begin to develop 'pseudopodia', with which they migrate in between epiblast and hypoblast, thereby creating a new layer, the intraembryonic mesoderm ${ }^{8}$.

From now on, while the mechanism called 'gastrulation' takes place, the epiblast is called the ectoderm, the hypoblast is called the endoderm and the embryo consists of three layers: ectoderm, mesoderm and endoderm (Figure 1.2).

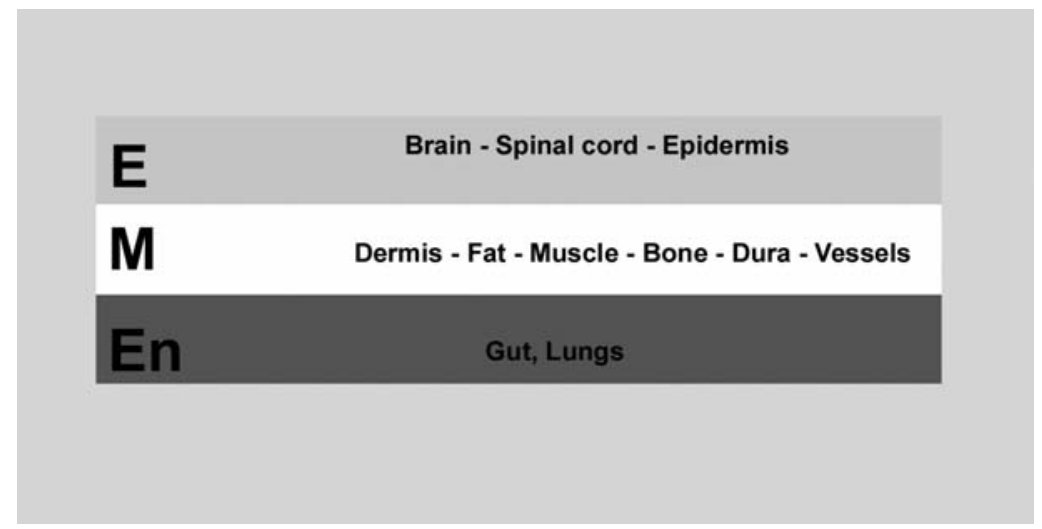

Figure 1.2 $E=$ ectoderm, $M=$ mesoderm, $E n=$ Endoderm.

See page 154 for colour figure.

From the ectoderm, the brain and spinal cord come to development, as well as the epidermis. The mesoderm gives rise to all structures surrounding the central nervous system (meninges, bones, muscles, blood vessels) and it is from this layer that the dermis and the subcutaneous fat have its origin. The lungs and gut develop from the endoderm. However, all three layers are closely associated and express interlayer inducing factors in many developmental processes.

\section{Objectives of this thesis}

A tethered cord, or a patient presenting with a tethered cord syndrome is a frequently encountered problem for the paediatric neurosurgeon. In many cases, the etiology of this tethering is rather well understood, and the patient can be successfully treated by adequate surgical untethering. 
However, some patients present with more complex pathology and in those cases a thorough understanding of the anatomical configuration of such malformations, combined with knowledge of their embryological etiology, is of significant importance to the surgeon.

The aim of this thesis is to describe several complex tethering mechanisms, focussing on clinical, embryological, anatomical, radiological and surgical landmarks, in order to extend the knowledge of the paediatric neurosurgeon dealing with these tethered cord malformations.

\section{Outline of the thesis}

- In chapter 2, the anatomy of a fetal myelomeningocele at 20 weeks' gestation is shown, using high-field magnetic resonance imaging.

- In chapter 3, the anatomy and surgery of the infected dermal sinus of the lower spine are described.

- In chapter 4, a new entity, the dermal sinus-like stalk is introduced. Embryological, anatomical and surgical aspects of this variation on the dermal sinus are explained.

- In chapter 5, results are shown of an experimental study in which a dermal sinus is induced in chick embryos.

- In chapter 6, the embryology, anatomy and surgery of the split cord malformation are discussed.

- In chapter 7, the surgical management of the tethered cord in patients presenting with a Currarino triad is discussed.

- In chapter 8, results are provided of a series of intraspinal dermoid and epidermoid tumors. Embryological, anatomical, clinical and surgical findings are presented.

- In chapter 9, the findings of the thesis are brought into perspective. Neurosurgical implications are discussed and an overall conclusion is provided.

The research for this thesis was performed at the Departments of Neurosurgery and Child Neurology of the Maastricht University Medical Center, the Department of Anatomy/Embryology of Maastricht University, and GROW (School for Oncology \& Developmental Biology) Maastricht University, The Netherlands. 


\section{References}

1. Barson AJ. The vertebral level of termination of the spinal cord during normal and abnormal development. J Anat 1970;106:489-497.

2. Brezner A, Kay B. Spinal cord ultrasonography in children with myelomeningocele. Dev Med Child Neurol 1999;41:450-455.

3. Bruner JP, Tulipan N, Paschall RL, Boehm FH, Walsh WF, Silva SR, Hernanz-Schulman M, Lowe LH, Reed GW. Fetal surgery for myelomeningocele and the incidence of shuntdependent hydrocephalus. JAMA 1999;282:1819-1825.

4. Bui CJ, Tubbs RS, Oakes WJ. Tethered cord syndrome in children: a review. Neurosurg Focus 2007;23:1-9.

5. Dubreuilh MW. Abces Intra-medullaire consecutif a une tumeur dermoide congenitale. Bull Soc Anat Physiol Norm Pathol 1887;8:13-26.

6. Kang JK, Kim MC, Kim DS, Song JU: Effects of tethering on regional spinal cord blood flow and sensory-evoked potentials in growing cats. Childs Nerv Syst 1987;3:35-39.

7. Kocak A, Kilic A, Nurlu G, Konan A, Kilinc K, Cirak B, Colak A. A new model for tethered cord syndrome: a biochemical, electrophysiological, and electron microscopic study. Pediatr Neurosurg 1997;26:120-126.

8. Larsen WJ, Sherman LS. Human embryology, ed 3rd. New York, N.Y., etc.: Churchill Livingstone, 2001

9. Lew SM, Kothbauer KF. Tethered cord syndrome: an updated review. Pediatr Neurosurg 2007;43:236-248.

10. McLone DG (ed). Pediatric Neurosurgery: Surgery of the developing nervous system, ed 4th. Philadelphia: W.B. Saunders Company, 2001

11. Moise TS. Staphylococcus meningitis secondary to a congenital sacral sinus. Surg Gynecol Obstet 1926;42:394-397.

12. Ozek MM, Cinalli G, Maixner MJ (eds). Spina Bifida. Milan, Italy: Springer-Verlag Italia, 2008

13. Pang D. Split cord malformation: Part II: Clinical syndrome. Neurosurgery 1992;31:481-500.

14. Pang D, Dias MS, Ahab-Barmada M. Split cord malformation: Part I: A unified theory of embryogenesis for double spinal cord malformations. Neurosurgery 1992;31:451-480.

15. Ripley W, Thompson DC. Pilonidal sinus as a route of infection in a case of staphylococcus meningitis. Amer J Dis Child 1928;36:785-788.

16. Schneider SJ, Rosenthal AD, Greenberg BM, Danto J. A preliminary report on the use of laser-Doppler flowmetry during tethered spinal cord release. Neurosurgery 1993;32:214-217, discussion 217-218.

17. Shenkin HA, Hunt AD, Horn RC. Sacrococcygeal sinus (pilonidal sinus) in direct continuity with the central canal of the spinal cord. Surg Gynecol Obstet 1944;79:655-659.

18. Tani S, Yamada S, Knighton RS. Extensibility of the lumbar and sacral cord. Pathophysiology of the tethered spinal cord in cats. J Neurosurg 1987;66:116-123.

19. Tulipan N. Intrauterine myelomeningocele repair. Clin Perinatol 2003;30:521-530.

20. Tulipan N, Sutton LN, Bruner JP, Cohen BM, Johnson M, Adzick NS. The effect of intrauterine myelomeningocele repair on the incidence of shunt-dependent hydrocephalus. Pediatr Neurosurg 2003;38:27-33.

21. Walker E, Bucy PC. Congenital dermal sinuses: a source of spinal meningeal infection and subdural abscesses. Brain 1934;57:401-421.

22. Yamada S (ed). Tethered Cord Syndrome. Park Ridge, Illinois, USA: The American Association of Neurological Surgeons, 1996

23. Yamada S, Zinke DE, Sanders D. Pathophysiology of "tethered cord syndrome". J Neurosurg 1981;54:494-503. 


\section{In vitro high-field magnetic resonance imaging-documented anatomy of a fetal myelomeningocele at 20 weeks' gestation. A contribution to the rationale of intrauterine surgical repair of spina bifida}

Emile A.M. Beuls, Linda Vanormelingen, Jasper van Aalst, Marjan Vandersteen, Peter Adriaensens, Erwin M.J. Cornips, Johan S.H. Vles, Jan Gelan

Journal of Neurosurgery: Spine 2003;98(2 Suppl): 210-214 


\section{Abstract}

\section{Object}

It remains uncertain if closure of a myelomeningocele at midgestation changes the neurological condition at birth in an infant born with spina bifida. The authors conducted a study to provide a detailed analysis of the morphology of the spinal cord with a myelomeningocele at the time fetal surgery usually is performed.

\section{Methods}

The myelomeningocele of a 20 -week-gestation-age fetus was examined and data were compared with those obtained in a neurologically intact specimen of the same age. In vitro high-field 9.4-Tesla magnetic resonance (MR) microscopy was used to examine the fetal material.

High field MR spectroscopy provided images in the three orthogonal planes with a resolution comparable with low-power optical microscopy. The authors observed that the fetal cord of the myelomeningocele specimen was tapered and tethered at S3-4 while the conus medullaris in the normal fetus reaches $L 4$.

No neurulation defects were noted. The axial MR images clearly revealed the non-fusion of the mesodermal structures. The absence of neurulation defects suggests that at least in some cases of spina bifida the spinal cord initially is well developed but is damaged later on chemically and mechanically. This might be an argument in favor of intrauterine myelomeningocele repair. By 20 weeks' gestation, however, the deformation of the cord inside the myelomeningocele is severe. An optimization of the preoperative assessment by means of MR imaging therefore might be considered a valuable contribution to intrauterine surgery. The in vitro high-field MR microscopic findings of this study could be used as references for clinical intrauterine MR imaging.

\section{Conclusions}

The detailed in vitro high-field MR analysis of a 20-week-gestation-age fetus with spina bifida demonstrated that an improvement of the preoperative intrauterine imaging should be pursued to detect those cases without neurulation defects and with minimal deformation of the spinal cord. 


\section{Introduction}

Despite encouraging results obtained in animal studies, the initial goal of midgestational closure of a myelomeningocele to prevent neurological deficits at birth is not entirely fulfilled ${ }^{5,7,8,12-14,16}$.

The origin of myelomeningocele, the most common neurological malformation of the human newborn, is largely unknown. For a long time, a myelomeningocele was considered to be the result of a dysgenetic disorder involving the primary or secondary neurulation mechanism.

The authors of recent studies in human fetuses with a myelomeningocele, however, have suggested that in many if not all cases an initially normally developed cord remains exposed because the overlying mesodermal structures fail to close $e^{6-8,11}$. This may lead to a cascade of secondary damage responsible for the observed neurological deficits at birth. The exposed cord can be damaged by chemical and mechanical factors.

Chemical damage is provoked by the toxic influence of amniotic fluid on the developing nerve tissue. Progressive accumulation of cerebrospinal fluid in an arachnoidal pouch ventral to the cord, causes stretching and protrusion of the $\mathrm{cord}^{6}$. The rationale for intrauterine closure of the myelomeningocele is to cover the exposed cord as early as possible in order to prevent further chemical and mechanical damage to the $\operatorname{cord}^{6-9,12-14}$. To evaluate the morphological changes of the cord inside the myelomeningocele defect at the time intrauterine surgery is planned, the microanatomy of the myelomeningocele area of an aborted fetus of 20 weeks' gestation with spina bifida fetus was analyzed and compared to the lumbosacral region obtained in a 21-week-old human fetus without any overt neurodevelopmental defects.

Because the fragility of the nerve tissue makes macroscopic dissection difficult ${ }^{1,10,17}$, high field 9.4-Tesla MR imaging was chosen to examine the fetal specimens. It provides images in the three orthogonal dimensions, with an inplane pixel resolution comparable with low power optical microscopy. This method has been proven to be most valuable in the examination of an adult nerve specimen ${ }^{2,3}$. 


\section{Material and methods}

Postmortem investigation was performed in a medicolegally aborted human fetus at 20 weeks of gestation. Prenatal ultrasonographic investigation revealed a large lumbosacral myelomeningocele. Clinical 1.5-Tesla MR imaging performed immediately postabortion demonstrated the lumbosacral defect but the myelomeningocele cyst was not observed (Figure 3.1). An Arnold-Chiari type II malformation was demonstrated as well.

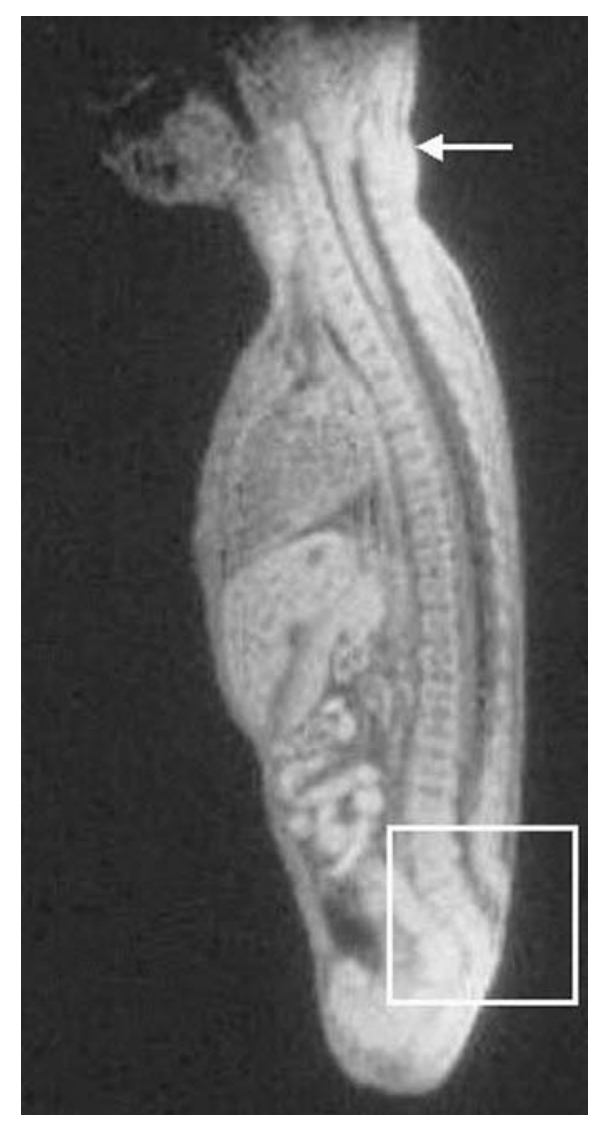

Figure 2.1 Midsagittal 1.5-Tesla MR image (TR 1800msec, TE $30 \mathrm{msec}$ ) of the 20-week-old fetus with spina bifida obtained immediately postabortion. An Arnold-Chiari Type II malformation is demonstrated at the craniocervical region (arrow). The spinal defect can be seen in the lower lumbosacral area. The cystic pocket visualized on prenatal ultrasonography cannot be seen. 
The fetus was fixed in $2 \%$ formalin solution. Subsequently high-field MR images of the thoracic and lumbosacral regions were obtained using a multisection spin-warp technique on a commercial Varian (Varian Nuclear Magnetic Resonance Instrument, Palo Alto, CA) Unity (9.4-Tesla) 400 spectrometer, equipped with an inner probe diameter of $25 \mathrm{~mm}$. The limiting probe dimensions were $25 \mathrm{~mm}$ in diameter and $40 \mathrm{~mm}$ in height according to the height of the coil homogeneity. Slightly T2-weighted spin density images (TR. $2500 \mathrm{msec}$, TE. $40 \mathrm{msec}$ ) were generated in the three orthogonal planes. The number of transients was 32 for transverse images and 16 for longitudinal images, resulting in an acquisition time of four tot eight hours for the collection of slices in each direction per sample. Slice thickness was $1 \mathrm{~mm}$. For transverse images the field of view was 17 by $17 \mathrm{~mm}$ with a matrix size of 350 by 50; longitudinal image parameters were 38 by $17 \mathrm{~mm}$ with a data matrix size of 700 by 320 . Pixel resolution was 40 by 40 micron.

High-field MR images obtained in the fetus with spina bifida were compared with those acquired in a 21-week-old fetus obtained after cesarean section performed after a life-threatening placenta praevia-induced hemorrhage. This specimen was fixed in $8 \%$ formalin administrated through an umbilical catheter after the autopsy revealed no underlying neurological disease. The high-field MR investigation of this fetus was performed by the same method, after which neuropathological examination (standard Masson trichrome staining) was performed to confirm structure identification.

\section{Results}

At the level above the myelomeningocele the high-field MR imaging (Figure 2.2 upper left and right) demonstrated that the spinal nerve rootlets and the meningeal coverings are comparable with those of the normal fetal spinal cord (Figure 2.3 lower left and right). The nervous tissue of the pathological specimen generally showed increased signal intensity and reduced contrast enhancement. The central canal is open, the spinal nerve roots have an oblique downward course to their corresponding intervertebral foramina, and the subarachnoid space in the spina bifida fetus is reduced. 

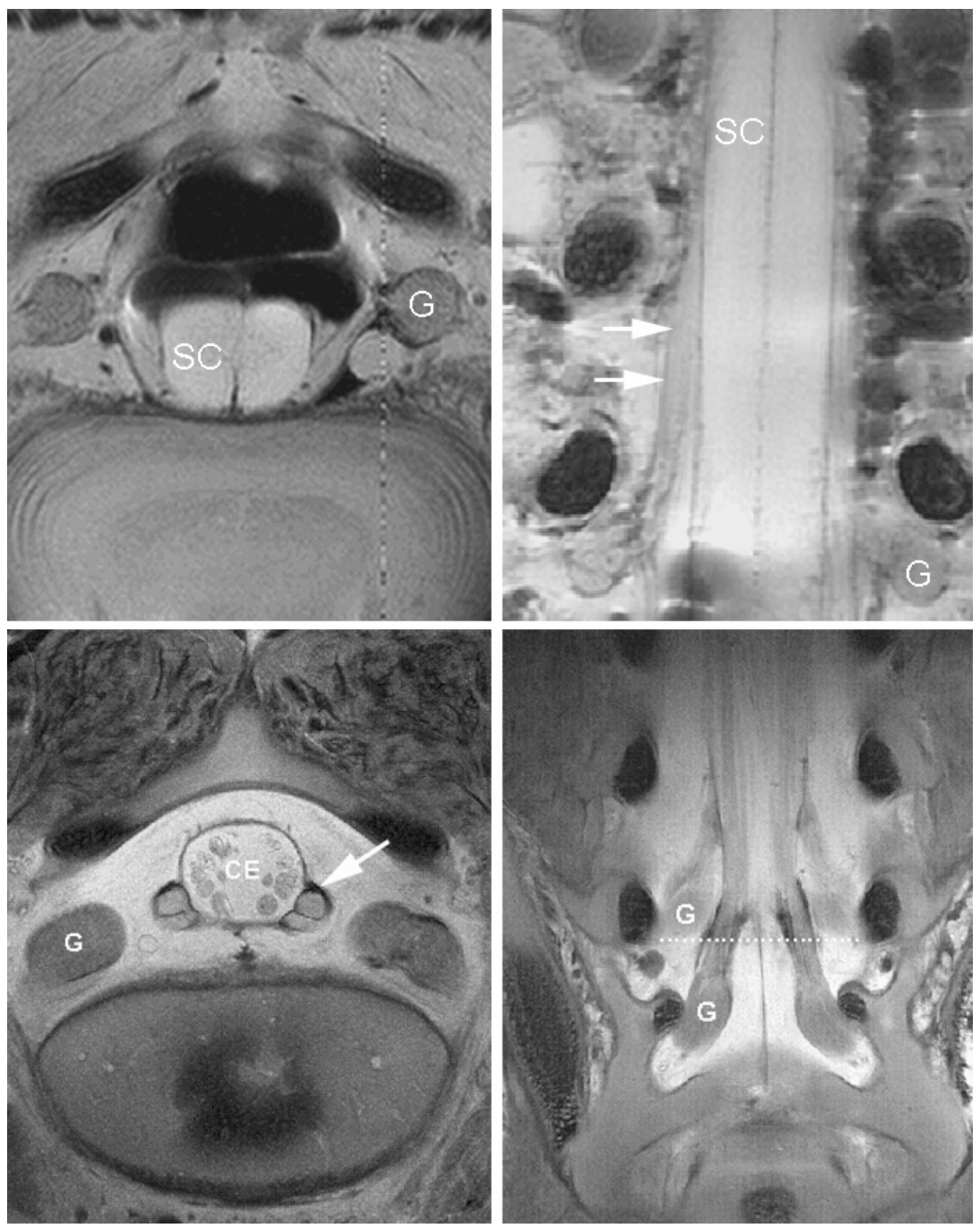

Figure 2.2 Upper left and Right: In vitro high-field MR (9.4-Tesla) axial (left) and coronal (right) images at the thoracic lumbar junction in the 20-week-old fetus with spina bifida. Down to the upper level of the myelomeningocele defect the spine, the spinal cord (SC), the nerve roots, and the corresponding ganglia show a normal configuration. On the coronal image (upper right) the long oblique course of a nerve root (white arrows) toward its corresponding upper lumbar spinal ganglion is demonstrated.

Lower Left and Right: In vitro high-field MR images of the neurologically healthy fetus (21 weeks of gestation). Lower Left: Axial image of S1 demonstrating the cauda equina (CE) surrounded by dura mater. At both sides clearly separated dorsal and ventral spinal nerve roots leave the dural sac (arrow). Lower Right: Coronal image obtained through the cauda equina at the lumbosacral junction, demonstrating the branching of the consecutive nerve roots as well as their corresponding spinal ganglia $(G)$ at the entrance of the intervertebral foramina. 
At the level of the myelomeningocele, consecutive axial images (Figures 2.3, 2.4) demonstrated the morphological changes of the vertebrae, the leptomeningeal membranes and the spinal cord. At the 4th lumbar vertebral level the anatomical configuration of the spine is normal. At the level of the 5th lumbar vertebra the laminae are bifid but the ossification center is present. At levels inferior to $L 5$ the paraspinal muscles were progressively separated from the midline. At the lower border of L4 the dura mater did not fuse in the dorsal midline but adhered to the muscle fascia bilaterally. The dorsal boundaries of the spinal cord became irregular. The size of the spinal cord progressively decreased. From the upper border of S1 downward, the dorsal surface of the cord was completely uncovered. In axial sections of the middle part of the defect, the nerve roots had a folded appearance. Sagittal images (Figure 2.5) revealed a slight enlargement of the spinal cord from the cranial border of the defect down to S2. The cord ends at the S4-S5 level. On coronal images the spinal nerve roots showed a slightly ascending course at the upper part of the defect whereas at the middle and lower parts of the cord their course was more horizontal. The nerve roots appeared to ascend again in the most caudal part (Figure 2.5). Spinal ganglia were present over the entire length of the spine, in thoracic as well as in the myelomeningocele regions. No cystic pocket was observed anterior to the spinal cord on either axial (Figures 2.3, 2.4), or longitudinal (Figure 2.5) high-field MR images. 

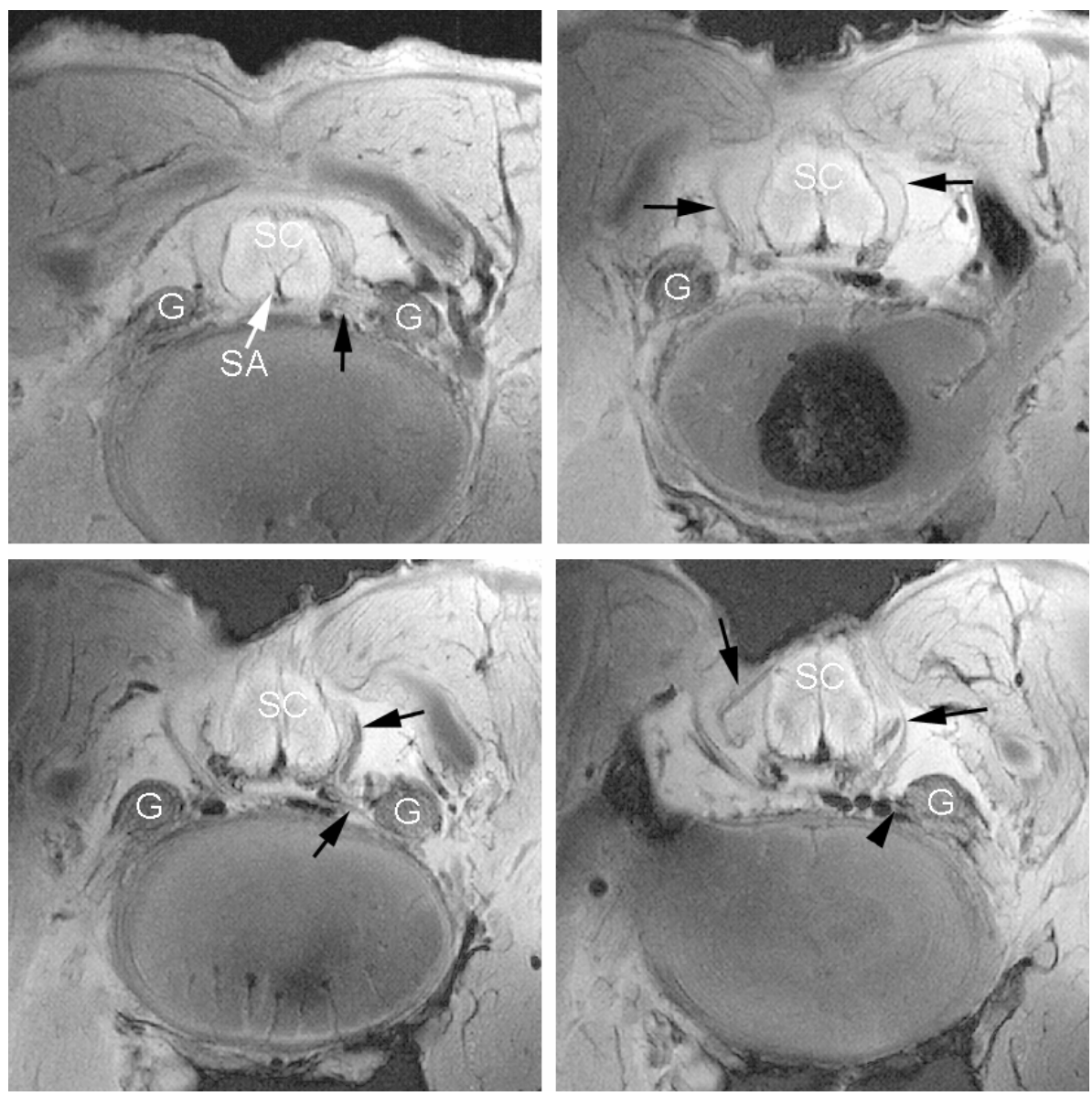

Figure 2.3 Consecutive axial high-field MR images obtained through the cranial part of the myelomeningocele defect (craniocaudal direction, from the upper left to lower right images) demonstrating the nonunion of the mesodermal and dermal tissues and the progressive exposure of the spinal cord (SC). At the lower border of L4 (upper left) the nonunion starts while the dura mater is closed. Further downward the cord is exposed by nonclosure of the overlying dura mater. The posterior surface of the cord is irregular. The size and shape of the cord are normal, without evidence of neurulation defects. The spinal ganglia $(G)$ are visualized at all levels. The folded appearance of the roots (black arrows) in the middle part of the defect (lower left) most probably is caused by collapse of the cystic pocket observed on prenatal ultrasonography. The anterior spinal artery (SA/white arrow) is shown in the upper left image. 

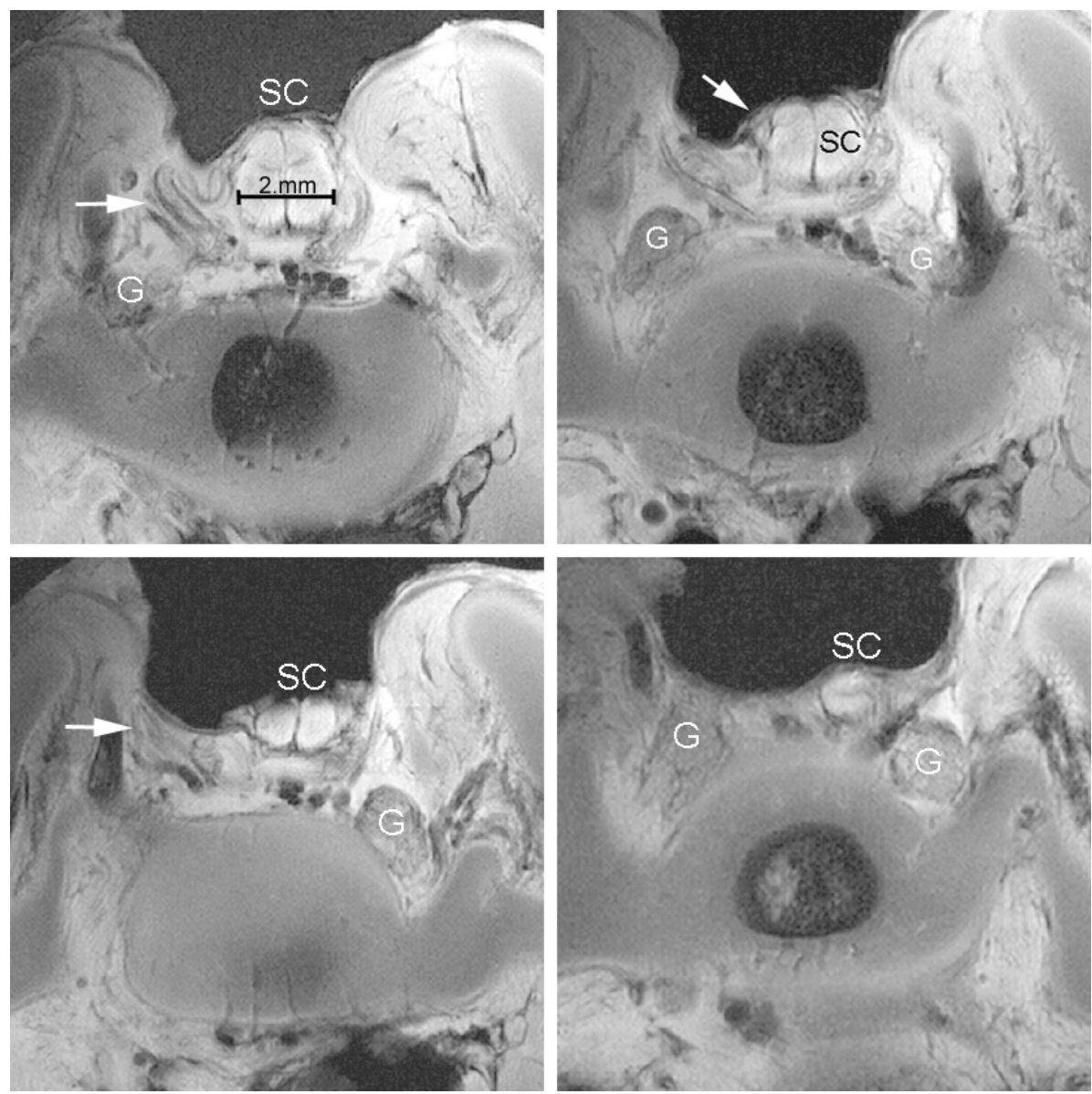

Figure 2.4 Subsequent four axial high-field MR images obtained through the myelomeningocele downward. A more pronounced folded appearance of the nerve roots (white arrows) is evident. Initially the myelomeningocele/spinal cord pushed outward resulting in the formation of a cystic pocket with straightening of the nerve roots. Because the cystic pocket collapsed during delivery, the spinal cord is displaced ventral and downward, leading to folding of the nerve roots. The size of the cord progressively decreases. Spinal ganglia are present up to the most caudal part of the cord. 

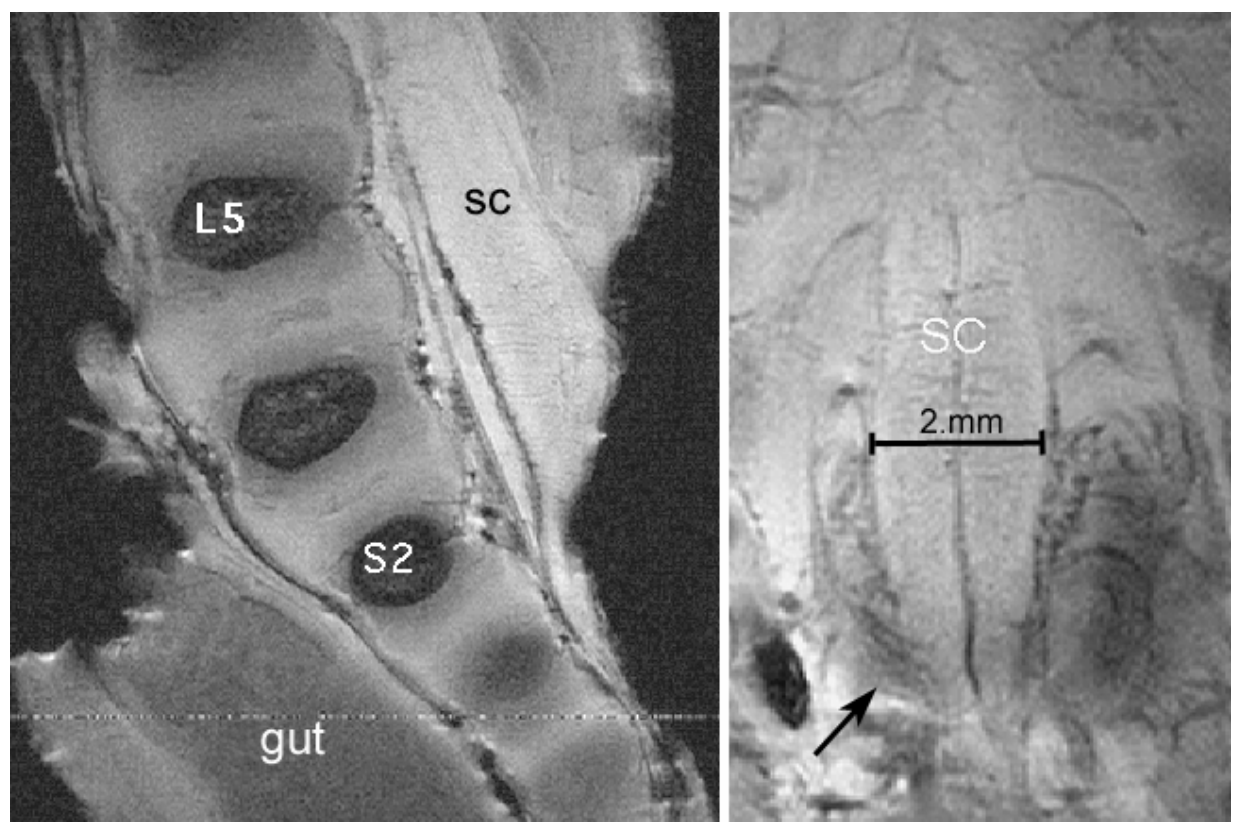

Figure 2.5 In vitro 9.4-Tesla high-field MR longitudinal images obtained at the level of the myelomeningocele of the 20-week-old fetus corresponding to the outlined area in figure 2.1.

Left: The sagittal section is not exactly midline. Because the specimen is positioned in a slightly oblique way, the cord initially appears enlarged and rapidly becomes flattened although gradually tapered. The cord extends tailwise downward to the lowest parts of the sacral canal. The mesodermal defect exceeds the trajectory of the exposed cord.

Right: Coronal image obtained through the long axis of the lowest part of the spinal cord inside the myelomeningocele defect revealing the taillike tapered extension of the conus medullaris ending as a small strip at the S4-S5 level. A bundle of rootlets with an ascending course is visualized in the lowest areas of the myelomeningocele defect (black arrow). 


\section{Discussion}

\section{Examination of fetal nerve tissue in normal and pathological conditions}

This investigation illustrates the usefulness of high-field MR imaging in the microanatomical analysis of fetal nerve tissues. In adult neuroanatomical specimen tissue contrast on high-field MR images is determined by the degree of myelination ${ }^{2,3}$. At 20 weeks of gestation myelin is hardly present in fetal nerve tissue and consequently cannot provide explanation for the observed contrast in fetal nerve tissue ${ }^{15}$. Moreover, fetal nonmyelinated white and gray matter have comparable high water content. As demonstrated on the slightly T2-weighted spin-density high-field MR images examined in this study, contrasting signal intensities between fetal gray matter and unmyelinated white matter were determined by the concentration and mobility of protons and as a consequence are an expression of the degree of structural tissue organization within the fetal spinal cord. The different signal intensities exhibited between the pathological and the normal fetal specimens might be correlated to the different formalin concentration used in the fixation of each specimen $(8 \%$ in the normal and $2 \%$ in the spina bifida fetus) because T2 signal relaxation is reduced by formalin ${ }^{4}$. In our opinion the images obtained by high-field MR imaging in vitro will provide valuable references for in vivo antenatal low field MR imaging.

\section{Absence of signs of neurulation defects}

On the high-field MR images no evidence of a primary neural nonclosure event can be demonstrated (Figures 2.3, 2.4). At the level of the myelomeningocele defect ventral and dorsal roots as well as spinal ganglia were present. This observation might support the hypothesis that at least in some cases of a myelomeningocele an initially well-developed cord becomes damaged by nonclosure of the overlying mesodermal and dermal structures ${ }^{6-8}$. It might also underscore the need for early antenatal closure of the defect to encourage more favorable results in cases in which the cord is without neurulation defects. In its role in patient selection for surgery, prenatal MR examination should therefore also be focused on demonstrating the state of development of the cord.

\section{Deformation of the spinal cord inside the myelomeningocele}

The different morphological aspects at the level of the defect are in accordance with a low position of the conus medullaris. On sagittal and coronal high-field MR images (Figure 2.5) the elongated and tapered cord was seen ending as a 
tiny strip below the S4-S5 level. At 20 weeks of gestation, it should have reached the L4 level, as was demonstrated on the high-field MR images obtained in the healthy fetus.

The low position of the conus was most probably caused by the formation of the cyst, which pushed out the spinal cord, resulting in straightening and elongation of the nerve rootlets.

The cyst, visualized on prenatal ultrasonography in this specimen, was no longer visible. The folded appearance of the nerve rootlets suggested a collapse of the cyst and a downward displacement of the spinal cord (Figure 2.4).

The images demonstrated that, despite the absence of intrinsic cord neurulation defects, the cord was severely deformed at the time the operation would be planned. In animal experiments tethering and deformation of the cord in early embryonic life have not been an issue, because the defect was artificially created at midgestation ${ }^{7,8}$. The results of these animal studies are therefore hardly comparable with those in the human myelomeningocele fetus. Simple closure of the myelomeningocele defects prevents further damage to the exposed cord. It seems unlikely that this procedure either could restore the ascensus of the cord or prevent further deformation by elongation.

Our observations support the assumption that MR imaging can be used to select those cases for which intrauterine surgery may be of benefit such as in patients without primary neurulation defects and also in those with minimal morphological deformations due to stretching of the cord. In this regard much will depend on the progress of the intrauterine MR imaging technology to match the in vitro high-field MR imaging resolution. The in vitro high-field MR imaging findings presented in this study could be used as references and extrapolated to the clinical setting involving intrauterine imaging.

\section{Conclusions}

At 20 weeks' gestation the fetal cord inside the myelomeningocele defect shows severe deformation but no signs of neurulation defects. The latter finding provides support for fetal surgery. Our in vitro high-resolution high-field MR imaging findings underscore that improvement in intrauterine imaging should be pursued to allow detection of cases without neurulation and with minimal spinal cord deformation. 


\section{References}

1. Bell JE, Gordon A, Maloney AF. The association of hydrocephalus and Arnold-Chiari malformation with spina bifida in the fetus. Neuropathol Appl Neurobiol 1980;6:29-39.

2. Beuls E, Gelan J, Vandersteen M, Adriaensens P, Vanormelingen L, Palmers Y. Microanatomy of the excised human spinal cord and the cervicomedullary junction examined with high-resolution MR imaging at 9.4 Tesla. AJNR Am J Neuroradiol 1993;14:699-707.

3. Beuls EA, Vandersteen MA, Vanormelingen LM, Adriaensens PJ, Freling G, Herpers MJ, Gelan JM. Deformation of the cervicomedullary junction and spinal cord in a surgically treated adult Chiari I hindbrain hernia associated with syringomyelia: a magnetic resonance microscopic and neuropathological study. Case report. J Neurosurg 1996;85:701-708.

4. Carey EM. The biochemistry of fetal brain development and myelination, in Jones CT (ed): Biochemical Development of the Fetus and the Neonate: Elsevier Biomedical Press, 1982:287-336

5. Hirose S, Farmer DL, Albanese CT. Fetal surgery for myelomeningocele. Curr Opin Obstet Gynecol 2001;13:215-222.

6. Meuli M, Meuli-Simmen C, Hutchins GM, Seller MJ, Harrison MR, Adzick NS. The spinal cord lesion in human fetuses with myelomeningocele: implications for fetal surgery. J Pediatr Surg 1997;32:448-452.

7. Meuli M, Meuli-Simmen C, Yingling CD, Hutchins GM, Hoffman KM, Harrison MR, Adzick NS. Creation of myelomeningocele in utero: a model of functional damage from spinal cord exposure in fetal sheep. J Pediatr Surg 1995;30:1028-1032; discussion 1032-1023.

8. Meuli M, Meuli-Simmen C, Yingling CD, Hutchins GM, Timmel GB, Harrison MR, Adzick NS. In utero repair of experimental myelomeningocele saves neurological function at birth. $J$ Pediatr Surg 1996;31:397-402.

9. Olutoye OO, Adzick NS. Fetal surgery for myelomeningocele. Semin Perinatol 1999;23: 462-473.

10. Peach B. Arnold-Chiari Malformation. Arch Neurol 1965;12:613-621.

11. Selcuki M, Manning S, Bernfield M. The curly tail mouse model of human neural tube defects demonstrates normal spinal cord differentiation at the level of the meningomyelocele: implications for fetal surgery. Childs Nerv Syst 2001;17:19-23.

12. Tulipan N, Bruner JP, Hernanz-Schulman M, Lowe LH, Walsh WF, Nickolaus D, Oakes WJ. Effect of intrauterine myelomeningocele repair on central nervous system structure and function. Pediatr Neurosurg 1999;31:183-188.

13. Tulipan N, Hernanz-Schulman M, Bruner JP. Reduced hindbrain herniation after intrauterine myelomeningocele repair: A report of four cases. Pediatr Neurosurg 1998;29:274-278.

14. Tulipan N, Hernanz-Schulman M, Lowe LH, Bruner JP. Intrauterine myelomeningocele repair reverses preexisting hindbrain herniation. Pediatr Neurosurg 1999;31:137-142.

15. van der Knaap MS, Valk J. Magnetic Resonance of Myelin, Myelination, and Myelin disorders, in. Berlin, Heidelberg, New York: Springer-Verlag, 1995:1-17

16. Walsh DS, Adzick NS, Sutton LN, Johnson MP. The Rationale for in utero repair of myelomeningocele. Fetal Diagn Ther 2001;16:312-322.

17. Woodward PJ, Sohaey R, Harris DP, Jackson GM, Klatt EC, Alexander AL, Kennedy A. Postmortem fetal MR imaging: comparison with findings at autopsy. AJR Am J Roentgenol 1997;168:41-46. 



\section{Chapter 3}

\section{Anatomy and surgery of the infected dermal sinus of the lower spine}

Jasper v Aalst, Emile A.M. Beuls, Erwin M.J. Cornips, Linda Vanormelingen, Marjan Vandersteen, Jacobiene W. Weber, Johan S.H. Vles

Childs Nervous system 2006; 22:1305-1315 


\section{Abstract}

\section{Object}

Cases of infected dermal sinus are scarce and detailed surgical anatomical descriptions are hardly found in literature. The clinical, radiological and surgical findings in four cases of an infected dermal sinus located at the lower spine are presented to elucidate the pathological anatomical configuration.

\section{Clinical Material}

The first case showed two dermal sinuses with a parallel course extra- and intradurally, ending in a confluence of cavities connected to the conus. In this case, as well as in the fourth case, the signs and symptoms were those of meningitis. The second case presented with meningitis and a subdural empyema, while the third case presented with an intradermoid-intramedullary abscess at the junction between the DS and the conus. This child probably showed signs and symptoms of conus involvement as early as during pregnancy.

\section{Conclusion}

The anatomy of the nervous elements in this congenital anomaly is heavily disturbed, more particularly in case of infection, due to extensive arachnoidal scarring. The latter renders dissection laborious and recognition of anatomical details difficult, resulting in complete excision of a dermal sinus in less than half of the cases. Despite their variability in presentation, most cases of an infected dermal sinus show similar, characteristic features. 


\section{Introduction}

A dermal sinus (DS) is a midline developmental anomaly along the neuraxis in which an epithelium-lined tract extends inwards from the skin surface for a variable distance ${ }^{2}$. A hairy patch inside its orifice, a hyperpigmented area, a hairy nevus or a capillary haemangioma is often associated ${ }^{9}$. The incidence is not exactly known, but is low. Only case reports as well as a few very small series are found in literature $e^{1,6,8,12-14,16}$.

The DS may present with great clinical and anatomical variability. In case of a spinal DS, it may end in the subcutaneous or muscular layer or continue through the laminae to find the dura and/or the underlying spinal cord. Also it can be seen terminating in a lipoma and even present associated with spina bifida aperta ${ }^{10,12}$. A quite variable connection with the central nervous system might be found, the conus being most frequently affected ${ }^{1,2,9,10,13,15}$. In case of extension of the DS through the dura, this tract serves as a pathway for the spread of infection to the central nervous system ${ }^{10}$ which is a serious neurological condition. It may occur in more than $60 \%$ of affected patients ${ }^{9}$ and most frequently presents with meningitis. Sometimes an abscess is found into an adjacent part of the central nervous system or even inside a dermoid tumor which is one of the associated features in $25 \%^{2,9}$.

Detailed information on the surgical anatomy of the DS is scarce. Surgery in these cases is difficult due to the complex anatomical configuration. Therefore the clinical, radiological and surgical findings in four cases of a lumbosacral infected DS are presented in this report. An identical configuration could be found and was further analyzed.

\section{Case 1}

\section{Clinical condition}

At the age of four months this child was referred to our hospital with a meningitis due to Peptococcus asaccharolyticus and Klebsiella oxytoca. Two paramedian dimples with a hairy tuft near the lumbosacral transition area at the right and left side of the midline were noted, some fluid discharge was seen. (Figure 3.1). 


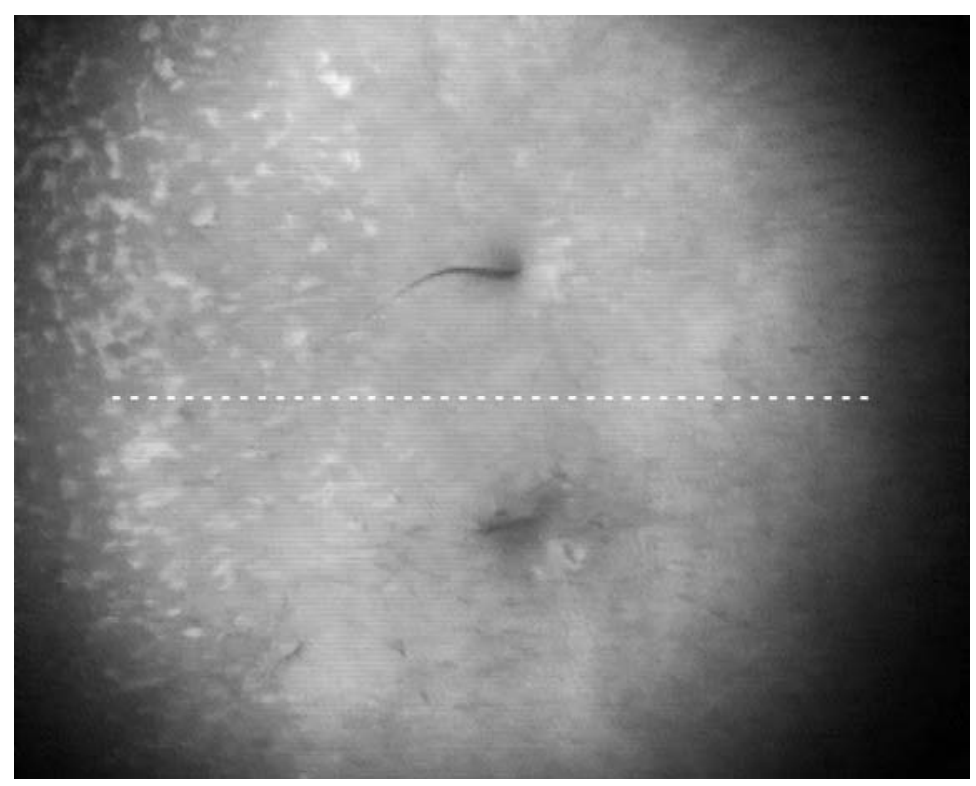

Figure 3.1 Double orifices at the skin surface, located at the right and left side of the midline (white dotted line). A characteristic tuft of hair is seen at both places (picture taken from low resolution videoregistration).

\section{Imaging}

Magnetic resonance (MR) imaging showed a subdural empyema dorsal to the cord, extending from the lower lumbar to the midthoracic area, with contrast enhancement of all membranes surrounding the cord and cauda. Two DS tracts extending from the skin through the dura and connected with the conus were noted. A dilation of both tracts ventrally connected to an area of pronounced enhancement, was interpreted as an area of infection or abscedation, closely adjacent to the ventral CSF space (Figure 3.2). On axial sections (Figure 3.3) the position of the conus was estimated at level L4-L5.

\section{Surgery}

The operation was performed after two weeks of antibiotic therapy. After opening the dural sac, thick arachnoidal membranes were seen covering the intradural structures. The parallel running tracts were carefully dissected out of the arachnoidal packing. Dilations of different size were found along their trajectory, filled with dermoid material and hairs as well as a yellow substance, interpreted as being old inflammatory material. The tracts ended in a conglomerate of cavities filled with typical dermoid material. Carefully, the ventral cerebrospinal fluid (CSF) compartment was opened. CSF was clear. 
The tracts were cut off from the conus where the lowest nerve roots were seen branching off in order not to damage vital nervous tissue. A kind of syrinx was still present inside the presumed conus. A packet of clumped nerve roots of the cauda equina lined the resection area (Figure 3.4).

No signs of infection, as suggested on MR imaging, were found. The postoperative course was uneventful.

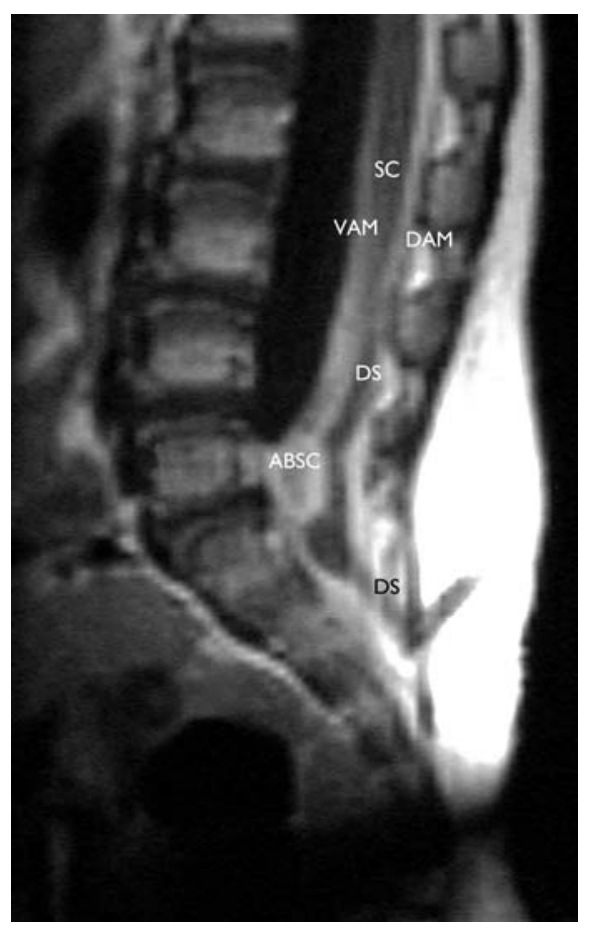

Figure 3.2 Sagittal paramedian MR T1 Gadolineum image, showing the trajectory of one of the two DS in case 1. A distention of the tract is seen. Intraoperatively, this mass proved to be filled with dermoid material. An presumably area of abscedation is seen above and adjacent to the dermoid tumor. The connection of the DS with the conus cannot be identified on this image. The skin orifice, the entrance into the dura and the connection with the conus are at different levels due to the embryological ascensus of the cord leaving the dura mater tube in place. DS: dermal sinus, ABSC: Abscess, SC: spinal cord, VAM: ventrally situated arachnoidal membranes, DAM: Dorsally situated arachnoidal membranes. 

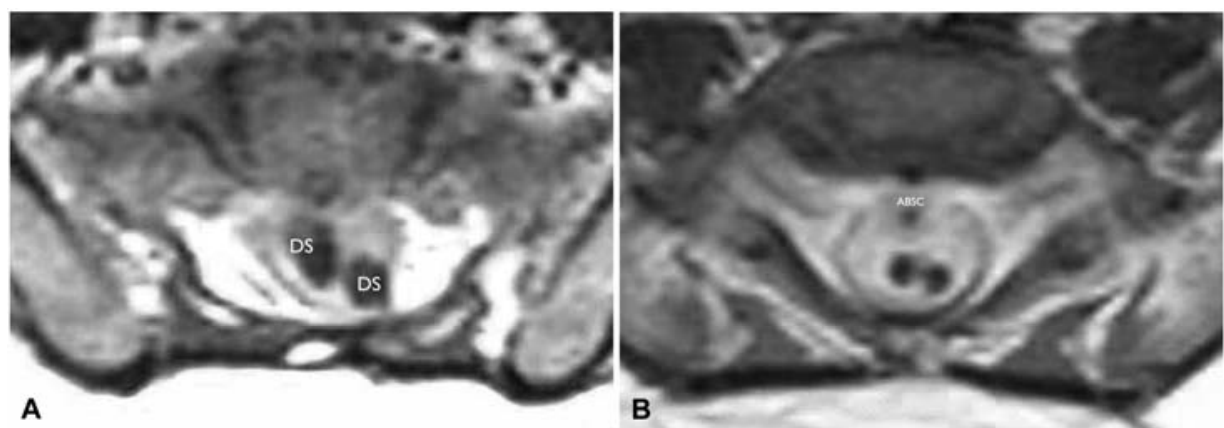

Figure 3.3 Consecutive Gadolineum enhanced axial MR T1 images show two DS at the same level (Case I). The corresponding longitudinal section is seen in Figure 3.2. A: The two DS converge to a parallel course at the entrance site into the dura mater. The low signal intensity inside the DS corresponds with fatty substance. B: Ventrally of the DS an area with a round configuration and heavily enhanced is seen which most probably corresponds to an abscess. The MR shows the two DS as well the abscess packed in thick arachnoidal membranes. DS: dermal sinus, ABSC: abscess.

\section{Case 2}

\section{Clinical condition}

At the age of nine months this otherwise healthy child was admitted to the hospital with a Proteus and Bacteroides meningitis. Neurological deficits were absent on clinical examination. The orifice of a previously unnoticed DS was found at midsacral level. As hydrocephalus developed, an external ventricular drainage was performed. After nine weeks of antibiotic therapy, infectious parameters had decreased and surgery was performed.

\section{Imaging}

MR imaging showed a large ventral subdural empyema, extending from midsacral up to low thoracic level. Also a mass, presumably epidurally situated at the level of the entrance of the DS into the dural sac was seen. Areas of infection were seen in surrounding extraspinal and intraspinal tissue layers. The caudal end of the conus could not be defined accurately, but was estimated at L4-L5. 


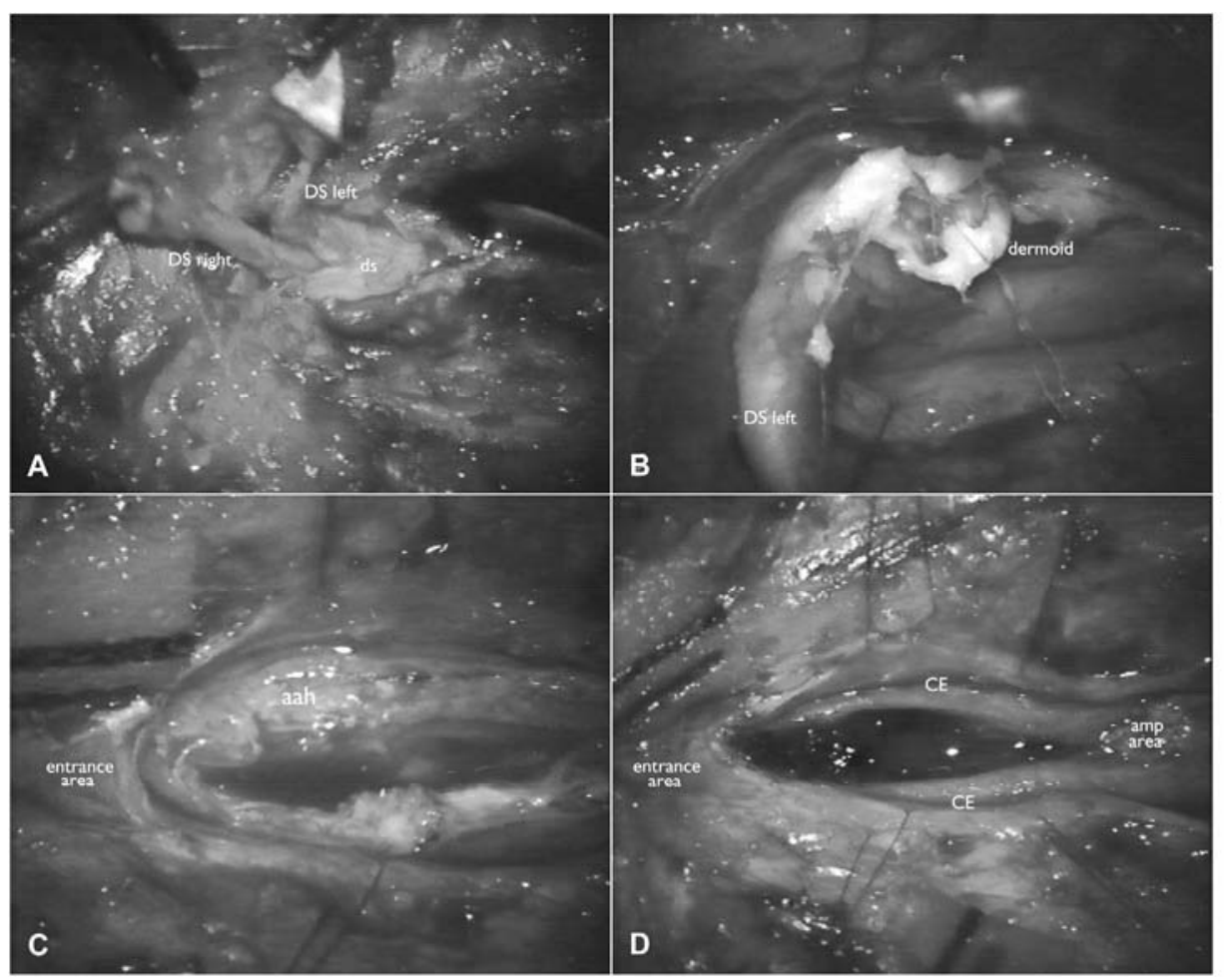

Figure 3.4 A: Dissection of the extradural trajectories of both DS out of the superficial layers. Both converge towards the midline to enter the dural sac at the same point. B: The lumina of both the DS are filled with an accumulation of dermoid material along its entire length. C: After removal of both the DS, an impressive package of the dense intradural arachnoidal membranes are left behind. The entrance side shows an identical configuration as seen in case 3 in which arachnoidal adhesions were absent. D: The cauda equina divided into two symmetrical packages of clumped nerve roots is dissected out of the arachnoidal membranes. The amputation area of both the DS from the conus has been performed at a safe distance from the lowest nerve roots. DS (or ds): dermal sinus, aah: arachnoidal adhesions, CE: cauda equina. Amp area: amputation area of the DS from the conus.

\section{Surgery}

The DS tract was dissected up to the dural sac, where dense granulation tissue of variable consistency surrounded its entrance into the dural sac. After opening the dura, the subarachnoidal space was obliterated as it was filled with firm arachnoidal adhesions surrounding the cauda, conus and spinal cord, even beyond the limits of the operative field. Despite careful dissection through these adhesions, the connection between the DS tract and the conus could not be identified. Some rootlets left the conus rather horizontally. A linear myelotomy at the supposed transition zone of the DS tract and the conus 
revealed an abundance of characteristic dermoid material and hairs, many of which were firmly attached to the inner walls of the dermoid cavity inside what was initially considered to be the spinal cord. The cavity continued upwards as far as could be seen through the limited myelotomy. Dissection of the cauda equina was subsequently carried out around the DS and the cord as far as possible ventrally to mobilize the DS block. An area at a distance of approximately $1,5 \mathrm{~cm}$ below the lowest nerve roots was chosen to cut of the DS. The cutting section showed that the DS and conus were lying on top of a tube filled with a milky fluid with white flocks. Conus and DS together with the empyema tube formed a single entity surrounded by a kind of shell consisting of tenacious arachnoidal membranes. The tube, being the empyema, was carefully canulated to rinse out gently as much debris as possible. The amputated DS part was dissected free and removed from the cauda equina roots.

From the dermoid debris a normal mixed skin flora was cultivated. Postoperatively the child did well without any neurological deficit except for a temporary urinary retention. His external ventricular drainage was internalized as his general condition improved rapidly.

\section{Case 3}

\section{Clinical condition}

At the age of 21 months this child was admitted to a regional hospital with a meningitis and urinary retention with signs of hydronephrosis of the left kidney in the absence of an urinary infection. Although the hydronephrosis had been diagnosed in the foetus during pregnancy, it disappeared after birth. No other neurological deficits were found during clinical examination. Two previously undetected dimples were found in the lumbosacral area, a typical sacral dimple and the orifice of a dermal sinus in the lower lumbar area. The child was treated with intravenous broad-spectrum antibiotics before it was transferred to our hospital.

\section{Imaging}

MR imaging demonstrated the entrance of the DS at the S2-S3 level continuing as a mass inside the conus up to level L2-L3. A small zone of contrast enhancement was seen in between the mass and the swollen conus (Figure 3.5). 


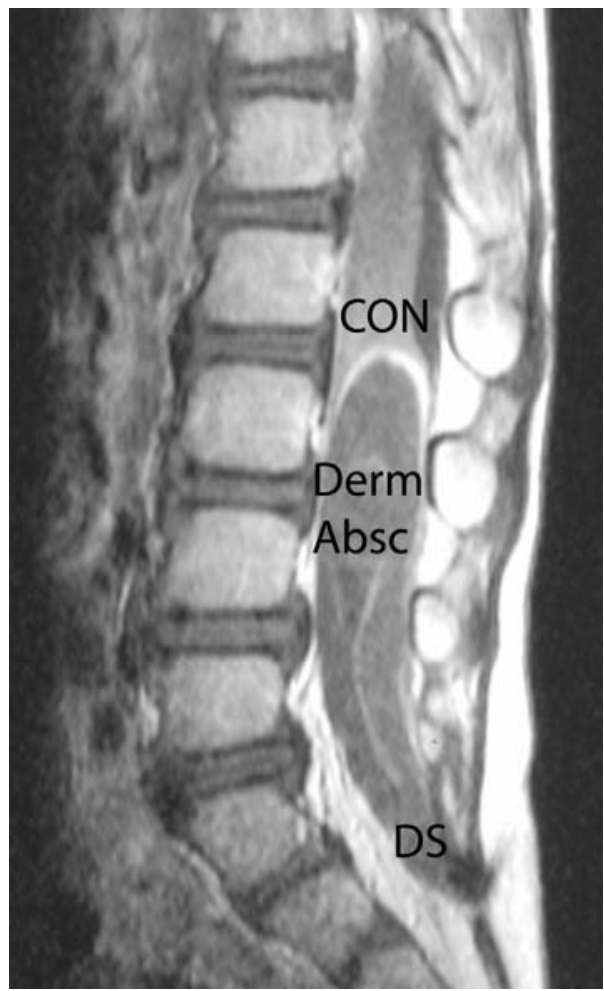

Figure 3.5 Sagittal T1 weighted MR Gadolineum enhanced image, showing the entire DS conus medullaris complex in Case 3. The intradural portion of the DS forms an anatomical continuum with the conus. The heterogeneous signal intensities correspond with accumulation of dermoid and infectious material. The high signal intensity capping of the intramedullary process suggests the presence of an inflammatory process most probably an abscess. The boundaries of the conus are non recognizable. DS: dermal sinus, Derm Absc: intradermoid abscess, CON: Conus medullaris.

\section{Surgery}

After nine weeks of antibiotic therapy and normalization of the infectious parameters, the child was operated. At careful dissection of the entrance site of the tract into the dural sac, a sleeve-like structure composed of a double layer of dura mater was found. The internal layer covering the tract over a short distance was only adherent to the tract and not fused with it. (Figure 3.6). 

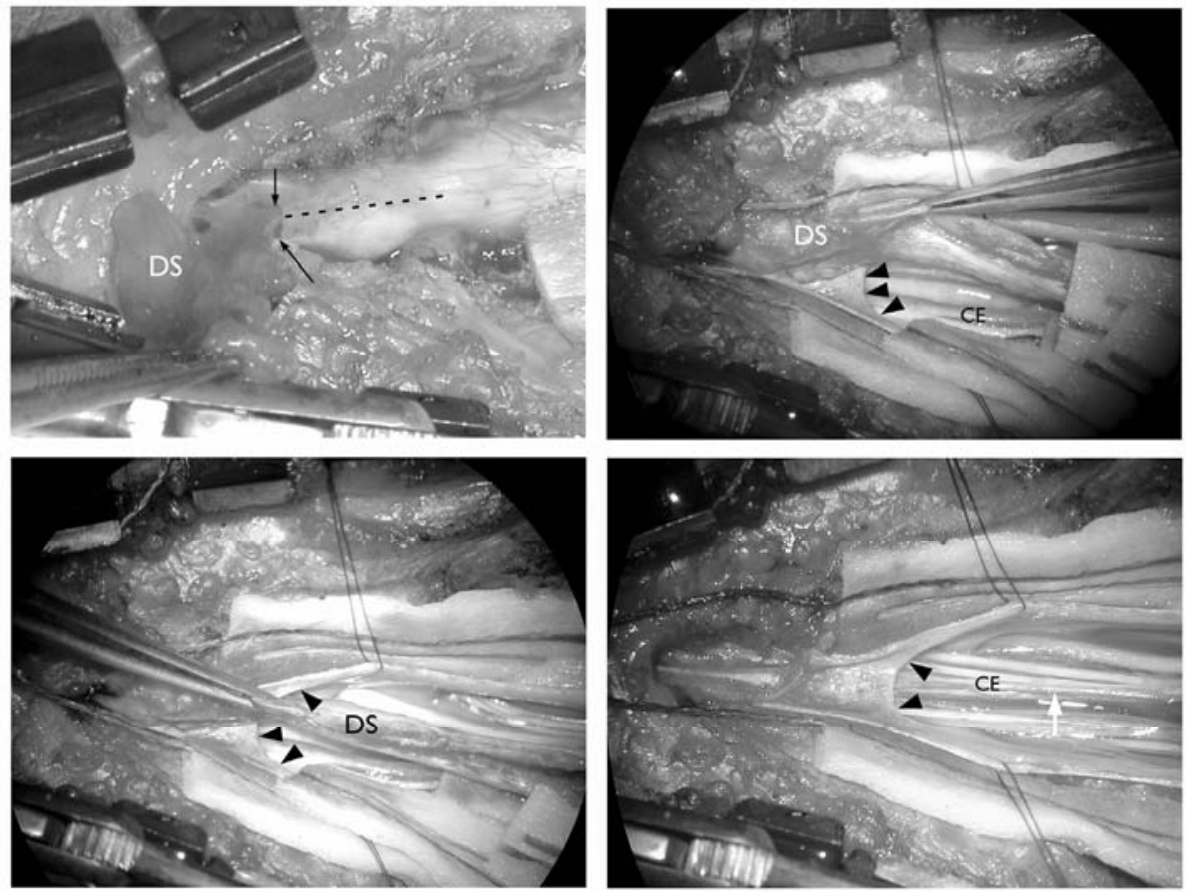

Figure 3.6 Intraoperative images of the entrance of the DS into the dural sac (Case 3).

Upper left. A cleaving plane may be seen at the site of entrance of the DS into the dura mater (black arrows). Along the dotted line the dural sac will be opened.

Upper right and under left. the intradural trajectory of the DS can easily be dissected out of a dural sleeve wrapped around the proximal portion of the intradural trajectory of the DS. Under right: a kind of a dural gutter is left after removal of the DS. Its distal border has the configuration of an arch (black arrow heads). DS and dural sleeve are only loosely connected with each other. After removal of the DS a filum terminale (white arrow) can be seen. DS: dermal sinus; CE: cauda equina

Normal arachnoidal membranes covered the entire intradural space, without arachnoidal adhesions. After a midline myelotomy over the swollen part of the cord at the presumed area of connection between DS and conus, liquid pus emerged under high pressure followed by the typical dermoid material with an abundance of hairs. Some hairs were tightly implanted into the walls of the cavity. Once the cavity had been emptied no dissection plane was found between its walls and the spinal cord. The DS was therefore amputated from the conus at a safe distance to the most caudal branching off rootlets. Once the cauda equina was fully exposed including the readily recognizable filum terminale the conus seemed satisfactorily untethered (Figure 3.7). Laboratory investigations failed to show microorganisms. 
The postoperative evolution was uneventful and urinary retention gradually resolved. The child was lost for follow-up.
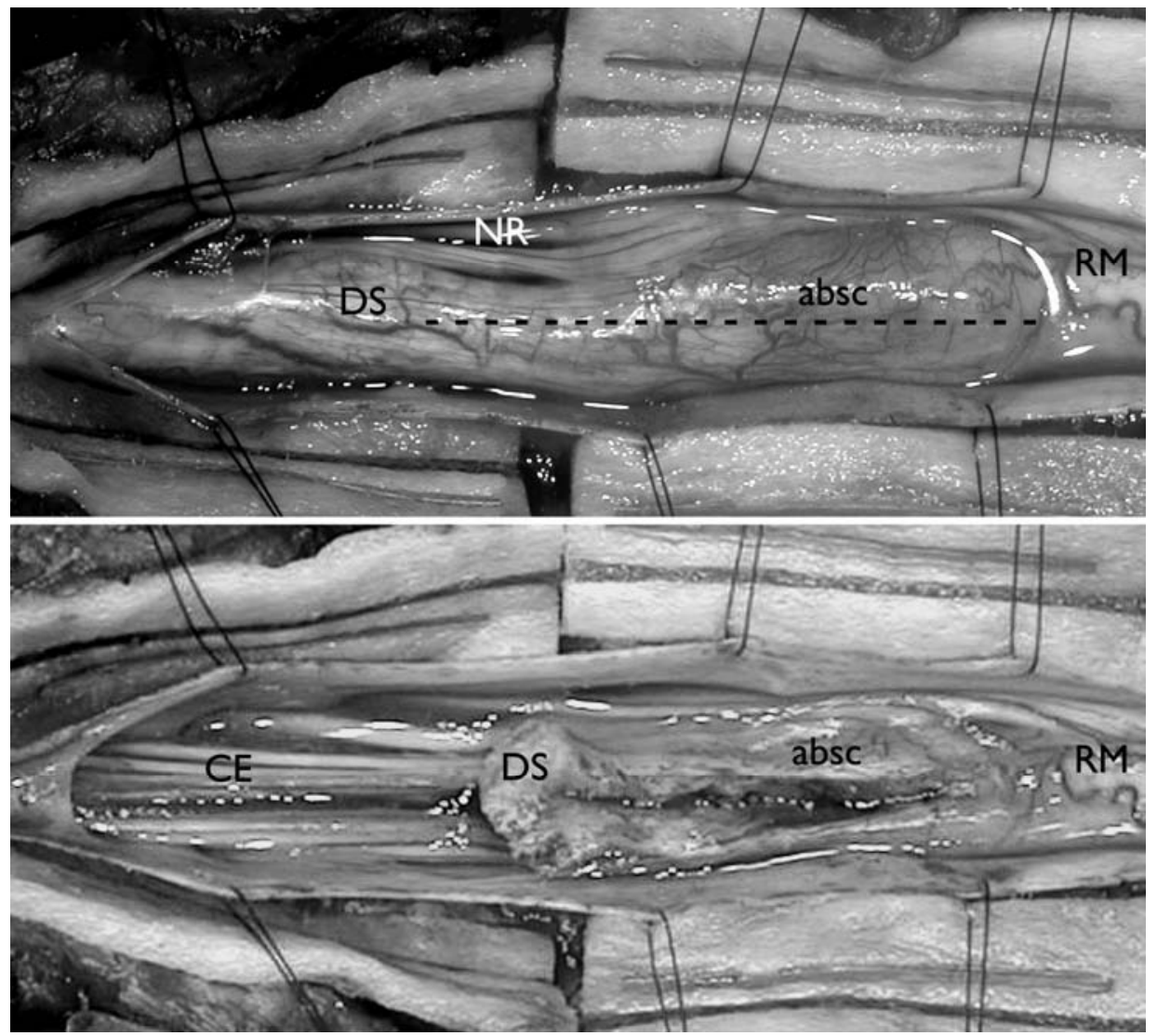

Figure 3.7 Intraoperative clinical pictures after opening of the dural sac in Case 3. The images can be compared with the corresponding longitudinal MR section (Figure 3.5). The upper image shows the connection between the DS and he spinal cord. An area of more localized swelling is seen and corresponds to the abscess. The dotted line shows the line of incision. There is an absolute absence of clear demarcation between the DS and the spinal cord. The lower image shows the open DS and conus after the evacuation of dermoid material and pus. The inner margins of the cavity are rather irregular and tough. The amputation of the DS was carried out at a safe distance of the lowest nerve roots exits. DS: dermal sinus, absc: abscess, NR: nerve roots of the cauda equina, RM: myelum (conus), CE: cauda equina, White arrow: filum terminale. 


\section{Case 4}

\section{Clinical condition}

At one month of age this child presented with a meningitis. No abnormalities were found at birth. The patient was treated with antibiotics, although cultures were negative. One year later, she was referred to our hospital because of intermittent fluid discharge from a small skin lesion, located at the median lumbosacral area.

\section{Imaging}

MR imaging showed a lenticular shaped mass, located intraspinally and presumably in the epidural space. This mass seemed to be connected to a tract continuing up to the skin lesion. The level of the conus was estimated at L1.

\section{Surgery}

The diagnosis infected dermal sinus was assumed and an explorative operation was performed.

After dissecting the trajectory from the cutaneous and subcutaneous layers, an infected pocket was found. It was not connected to the DS and consisted of inflammatory material, situated subcutaneously and in the paraspinal muscles. After removing this pocket, the tract was followed up to a distended part of it, which represented the mass, as noted on MR imaging.

The entire tract up to this distention was resected en bloc up to the connection of the tumor with the dura (Figure 3.8), which rather seemed an adherence instead of a connection. After resection, a small dural defect was found.

Some cerebrospinal fluid was appearing out of this defect. However, neither dural sleeve nor intradural continuation of the tract was seen. After opening the resected tumor, infected dermoid material appeared. The authors decided not to continue the exploration into the intradual space, due to the infected areas found in the more superficial layers. 


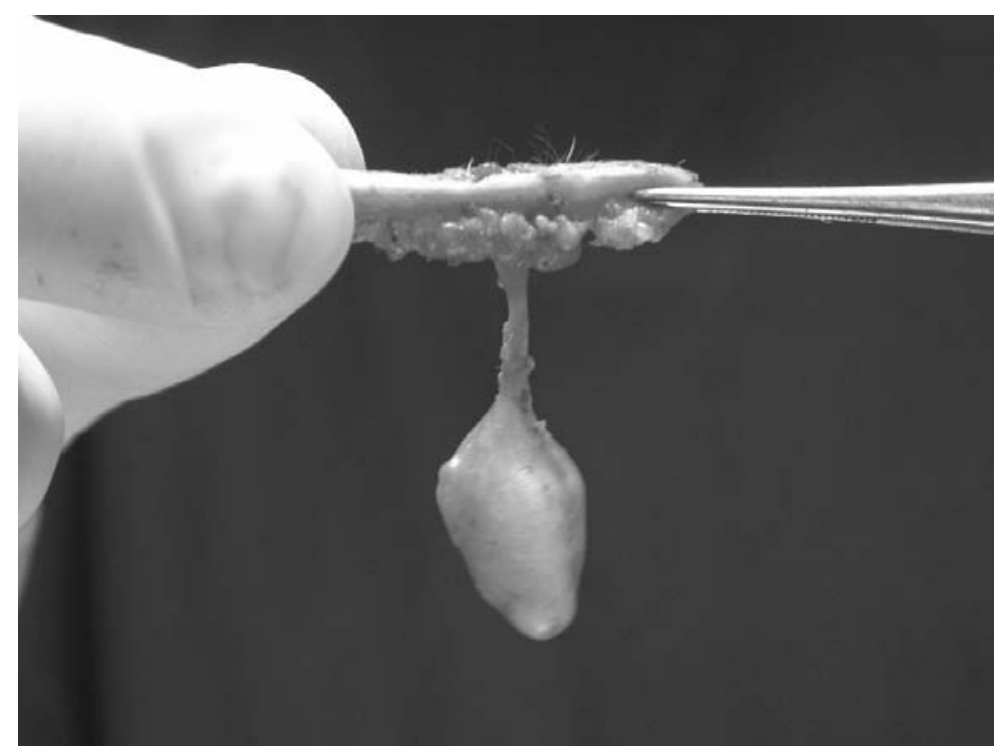

Figure 3.8 The specimen as resected in case 4. Note the distention of the tract giving rise to a mass. Intraoperatively, the mass contained infected dermoid material. This was confirmed by histological examination.

\section{Discussion}

\section{The incidence of the findings in general}

A single, paramedian tract ${ }^{9}$ and double midline tracts but at different levels ${ }^{3}$, have been described. Cases with two parallel DS tracts, positioned at the same level (case 1) have, to the best of our knowledge, not been reported. A subdural empyema associated with DS (case 2) has been described by Morandi et al. ${ }^{14}$. Reports of intratumoral and intramedullary abscesses (case 3 ) are scarce as well ${ }^{4,6,13,14}$. In a literature review Byrne et al reported only 14 cases of an intramedullary abscess secondary to a DS, only 3 of them were associated with an intramedullary dermoid tumor ${ }^{5}$.

In case 3 during pregnancy, a bilateral hydronephrosis was observed. If this had been interpreted as being due to damage to the conus, it could have led to early postnatal imaging followed by early surgery, which might have prevented the abscess. Although the presumption might remain speculative, it is to the best of our knowledge the first description of a sign of a DS during pregnancy. 
The anatomical configuration of the connection of the DS with the dura mater

All four cases had an identical anatomical configuration at the site where the DS was continuing intradurally. Because of the absence of arachnoidal adhesions this configuration was most purely observed in case 3 . The fistula approaches the dura and continues intradurally. The dura is accompanying the DS into the 'intradural' space by forming a sleeve around it, after its trajectory. When opening the sleeve and lifting up the DS, the DS loop seems to lie in a kind of gutter (Figures 3.6 and 3.9) with an arched margin.

It has been described that CSF appeared during surgery when cutting off the DS from the dura, which was explained as CSF leaking through the DS ${ }^{1}$. However, in our cases, this was not found. Although the inner dural layer is theoretically not hermetically attached to the DS it apparently can prevent CSF leakage from the subarachnoidal space. Possibly, an intact arachnoidal membrane can prevent leakage through the DS.

\section{The anatomy of the connection of the DS with the conus}

The further course of the DS in our three cases showed an identical configuration as well.

The DS follows a trajectory between the cauda equina roots for some distance until it merges with the conus without a clear transition zone (Figure 3.9). There seems to be a kind of anatomical continuum between conus and DS which should not be an uncommon finding ${ }^{2,10,15}$. Although the conus was obviously deformed, at the end of the dissection the cauda equina rootlets branching off symmetrically at both sides of the conus were clearly seen in the four cases. According to the authors, the latter can be an important landmark when determining where the DS will be sectioned from the conus.

\section{The so-called dermoid tumor}

Some reports describe the association of dermoid tumors with a DS as if the DS is connected to the dermoid tumor ${ }^{10}$. However the dermoid tumor merely develops inside the DS as a result of progressive accumulation of dermoid material formed by desquamation of the tract lining cells ${ }^{2}$. As reported by French ${ }^{9}$ and confirmed by our findings, a dermoid tumor may arise anywhere along a DS tract, at multiple sites within the same DS tract and even within the conus (case 3 ). 


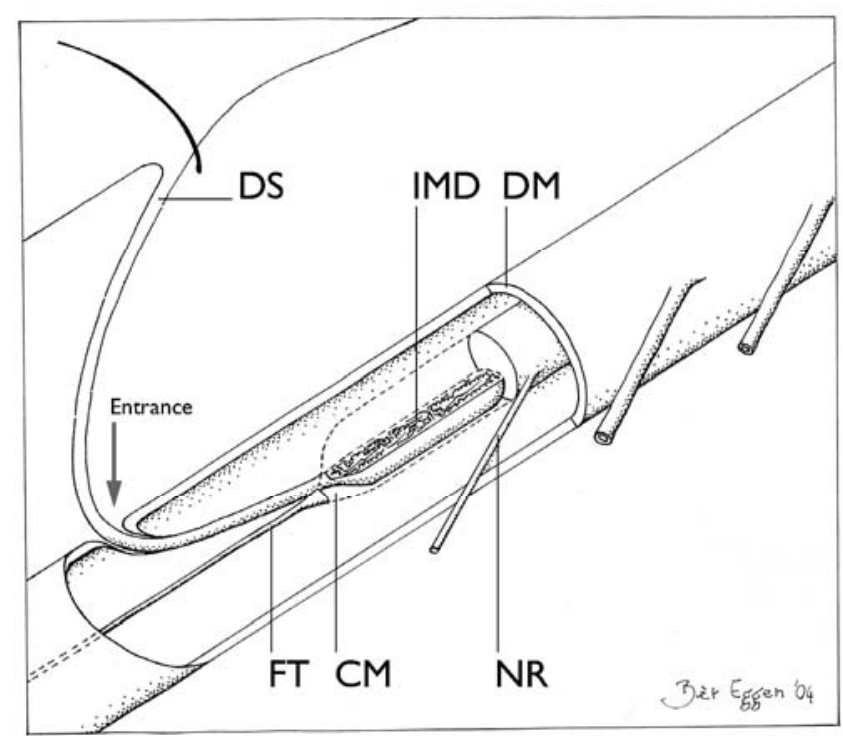

Figure 3.9 Schematic drawing of a characteristic DS-conus medullaris complex. The skin orifice, the site of entrance through the dura mater and the connection with the conus are situated at a different level. Due to the ascensus medullaris the orifice and the connection with the conus are located more cranially than the entrance point at the dura. The connection of the DS with the conus mostly forms a kind of anatomic continuum. A filum terminale might be present in most cases. DS: dermal sinus. FT: filum terminale. CM: conus medullaris. NR: nerve roots. IMD: intramedullary dermoid. DM: dura mater.

\section{The spread of the infection into the dural sac}

The spread of infection from the DS into the deep structures was different in our four cases. In case 1, 2 and 4 the infection spread into the subarachnoidal space (SAS) caused fulminate meningitis. Microorganisms might spread into the SAS through rupture of the distended walls of the DS, a process that might be comparable with the spread of fatty substances into the CSF causing a kind of Mollaret's chemicoleptomeningitis ${ }^{7,10}$. Massive infection of a sinus tract may cause local necrosis and abscess formation ${ }^{9}$ along the trajectory of the DS. In case 1 an intradural abscess formation was found, adjacent to an area of accumulation of dermoid material.

Case 2 displayed an infection located epidurally, in the deep extra- and paraspinal layers as well as subdurally.

Finally, in case 3 , an intramedullary abscess was suggested at the preoperative MR images. However during the operation the abscess proved to be rather inside the distended distal end of the DS than inside the cord itself. Bruff and Sgouros ${ }^{4}$ described a nearly similar case with presumption of an intramedullary extension. A case was reported with an intramedullary abscess 
inside a swollen conus in association with a DS presenting with a paraplegia ${ }^{3}$. The connection with the conus however is vaguely described.

It can be assumed that the spread of infection may follow any route along the course of the DS tract intra- as well as extradurally or a mixture of both.

\section{MR imaging in case of a DS}

MR imaging is of limited help in the preoperative assessment of an infected DS. The low position of the conus as well as an empyema or abscess might be visualized. However, interpretation of MR images of the transition area of the conus and the DS remains difficult. Barcovich et al. ${ }^{2}$ could visualize the connection of the DS tract with the conus in only two out of seven patients. In our series, only in case 1, the connection with the two DS complexes and the conus could be vaguely delineated. A T2-weighted intramedullary high signal area, cranial to a presumed abscess, may be a sign either of reactive oedema or of intramedullary extension of the abscess ${ }^{15}$. Accumulation of epidermoid or dermoid material may produce a quite inhomogeneous MR signal ${ }^{11}$, still more complex in case of infection. Thick arachnoidal adhesions and clumped nerve roots additionally complicate the interpretation of MR images ${ }^{2,14}$.

\section{Some surgical considerations}

To prevent recurrent infections as well as the recurrence of a dermoid tumor, the DS tract has to be completely excised ${ }^{4,14}$. However, due to firm adherences, only $40 \%$ of dermoid tumors can be excised ${ }^{14}$. But even in the absence of extensive arachnoidal adhesions it is notoriously difficult to determine the site of amputation of the DS tract from the conus. No clear recognizable dissection plane was present in any of our three patients. After meticulous dissection the amputation site was determined at some distance below the lowest nerve roots where it was estimated safe enough not to damage any part of the deformed conus. The en bloc excision of the DS tract ends somewhat artificially at this point.

The amputation or excision of the DS to prevent recurrence of infections in fact constitutes a preventive untethering. In case 3 however, a filum terminale was found between the cauda nerve roots after excision of the DS. The presence of a filum terminale could not be observed in the two other cases because of the clumping of the nerve roots of the cauda equina. If found intraoperatively, the area of attachment of the filum terminale may serve as another landmark in the anatomical continuum between conus and DS. 


\section{Conclusion}

Especially in cases of infection, understanding the unique anatomy of the DS and its connection with the conus is the key to a successful operative exploration preventing damage to conus and cauda. 


\section{References}

1. Ackerman L, Menezes A, Follett K. Cervical and thoracic dermal sinus tracts. Pediatric Neurosurgery 2002;37:137-147.

2. Barkovich AJ, Edwards M, Cogen PH. MR evaluation of spinal dermal sinus tracts in children. AJNR Am J Neuroradiol 1991;12:123-129.

3. Benzil DL, Epstein MH, Knucky NW. Intramedullary epidermoid associated with an intramedullary spinal abscess secondary to a dermal sinus. Case report. Neurosurgery 1992:30:118-121.

4. Bruff $P$, Sgouros S. Lumbar dermoid cyst causing pyomyelia in a child. Pediatr Neurosurg 2002;36:162-163.

5. Byrne RW, von Roenn KA, Whisler WW. Intramedullary abscess: a report of two cases and a review of the literature. Neurosurgery 1994;35:321-326; discussion 326.

6. Chen CY, Lin KL, Wang HS, Lui TN. Dermoid cyst with dermal sinus tract complicated with spinal subdural abscess. Pediatr Neurol 1999;20:157-160.

7. Crossley GH, Dismukes WE. Central nervous system epidermoid cyst: a probable etiology of Mollaret's meningitis. Am J Med 1990;89:805-806.

8. el-Gindi S, Fairburn B. Intramedullary spinal abscess as a complication of a congenital dermal sinus. Case report. J Neurosurg 1969;30:494-497.

9. French BN: Chapter 39; Congenital Spinal Dermal Sinus, in Youmans JR (ed). Neurological surgery: W.B. Saunders Company, 1990;2:1176-1182.

10. Friede RL: Developmental Neuropathology. Berlin: Springer, 1989.

11. Gupta S, Gupta RK, Gujral RB, Mittal P, Kuriyal M, Krishnani N. Signal intensity patterns in intraspinal dermoids and epidermoids on MR imaging. Clin Radiol 1993;48:405-413.

12. Jindal A, Mahapatra AK. Spinal congenital dermal sinus: an experience of 23 cases over 7 years. Neurol India 2001;49:243-246.

13. Maurice-Williams RS, Pamphilon D, Coackham HB. Intramedullary Abscess - a rare complication of sinal dysraphysm. J neurology, neurosurgery and Psychiatry 1980;43: 1045-1048.

14. Morandi X, Mercier P, Fournier H-M, Brassier G. Dermal sinus and intramedullary spinal cord abscess; Report of two cases and review of the literature. Child's Nervous System 1999; 15:202-207.

15. Tsurubuchi T, Matsumura A, Nakai K, Fujita K, Enomoto T, Iwasaki N, Nose T. Reversible holocord edema associated with intramedullary spinal abscess secondary to an infected dermoid cyst. Pediatr Neurosurg 2002;37:282-286.

16. Verdu Perez A, Montes Gonzalo MC, Alonso Martin JA. Gram-negative meningitis secondary to congenital dermal sinus demonstrated by magnetic resonance. An Esp Pediatr 1996; 44: 491-492. 


\section{Chapter 4}

\section{The spinal dermal sinus-like stalk}

Jasper van Aalst, Emile A.M. Beuls, Erwin M.J. Cornips, Henny W.M. van Straaten, Toon F.M. Boselie, Kim Rijkers, Jacobiene W. Weber, Johan S.H. Vles 


\section{Abstract}

\section{Object}

In this study a disjunction anomaly mimicking the spinal congenital dermal sinus (DS) is described. This anomaly is referred to as the dermal sinus-like stalk. Dissimilarities between a true dermal sinus and a dermal sinus like stalk are discussed.

\section{Clinical material}

Three cases in which a spinal congenital dermal sinus was suspected, are presented. A similar anatomical configuration, different from that of a dermal sinus was found. All cases presented with a skin-covered dimple from which a solid tract was seen continuing intramedullary in two cases and intraspinally in one case. None of the patients presented with signs of infection or an associated dermoid-epidermoid tumor.

Clinical, radiological, and surgical findings are discussed. A hypothesis is made on the pathological genesis of this malformation.

\section{Conclusions}

A dermal sinus like stalk is a malformation similar to a spinal congenital dermal sinus but is not associated with DS-related complications. Despite important clinical, radiological, surgical and histopathological differences, it is difficult to distinguish this malformation from a true DS based on clinical and radiological examination alone. Therefore, surgical intervention, at the time of diagnosis, is recommended in all cases. 


\section{Introduction}

A spinal congenital dermal sinus (DS) is a developmental anomaly of the dorsal midline axis, in which a hollow, epithelium-lined tract extends inward from the skin surface for a variable distance ${ }^{9}$. A key feature of a DS is an open skin defect. Occasional discharge from this orifice is a common finding. When a DS tract is surgically explored, a quite variable anatomical configuration is found. The outer lining of the tract is that of a white, smooth tube. The tract may end in the subcutaneous or muscular layers, but predominantly continues into the intradural space, where a delicate connection with the spinal cord is frequently found ${ }^{1,9,17}$. In many cases, the DS is continuous with the spinal cord at this point, and finding a dissection plane between both can be difficult ${ }^{17}$.

Lumbosacral dermal sinuses are usually associated with a low positioned conus medullaris and a tethered cord, with or without the clinical presentation of a tethered cord syndrome.

Another feature of a DS is the accumulation of dermoid-epidermoid remnants in the tract, often presenting as tumors inside the hollow tract or inside the spinal $\operatorname{cord}^{1,9}$.

Due to the open connection of the central nervous system with the surface of the skin, many cases present with meningitis and intraspinal, intramedullary or intra-epidermoid abscesses s-8,12,13,17. $^{5}$.

In this report the authors describe three cases of a tract mimicking a DS, but with significant differences. In all cases dimples were found, but in none of them an orifice was present. Moreover, the tracts did not have a lumen, nor did they contain dermoid-epidermoid remnants. However, all three tracts continued into the intradural space, and two ended in the spinal cord.

Although some authors mention tracts resembling the tracts described in our report ${ }^{10}$, to the best of our knowledge, a detailed description of such tracts has not been reported in the literature before. In order to differentiate these tracts from the true DS, the authors suggest to refer to this malformation as the spinal dermal sinus-like stalk. 


\section{Case 1}

\section{Clinical condition}

At birth a midline dimple, located at lower lumbar level and surrounded by a discoloration of the skin, was noted (Figure 4.1). A membrane covered the centre of the dimple. No neurological deficits were found at physical examination.

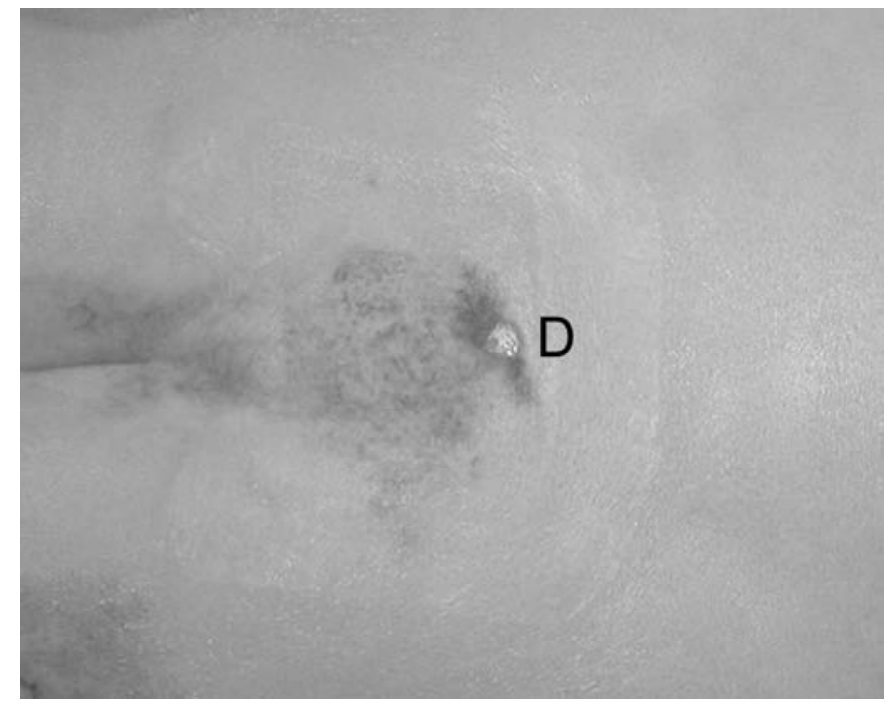

Figure 4.1 Intraoperative picture of dimple (D) in case 1.

\section{Imaging}

Magnetic resonance (MR) imaging showed a tethered cord with the level of the conus medullaris positioned at L4-L5. The dimple seemed to be attached to a tract, which was only vaguely depicted, continuing through the underlying tissues up to the conus medullaris.

\section{Surgery}

After circumscribing the dimple, a subcutaneous stalk was found, continuous with the skin. It did not resemble the white epithelium-lined tract as commonly found in a DS, but instead seemed to consist of dense fibrous tissue and fat. The stalk was seen continuing through the fascia and paraspinal muscles up to the dura mater. At that point, the stalk was surrounded by a dorsally directed 
dural sleeve, as is seen in case of a spinal nerve root. After opening the dura, a connection between the stalk and the conus medullaris in a typical DS-like way ${ }^{17}$ was found (Figure 4.2). The stalk was resected caudal to the lowest roots seen branching off the spinal cord. No lumen was detected in the resected specimen.

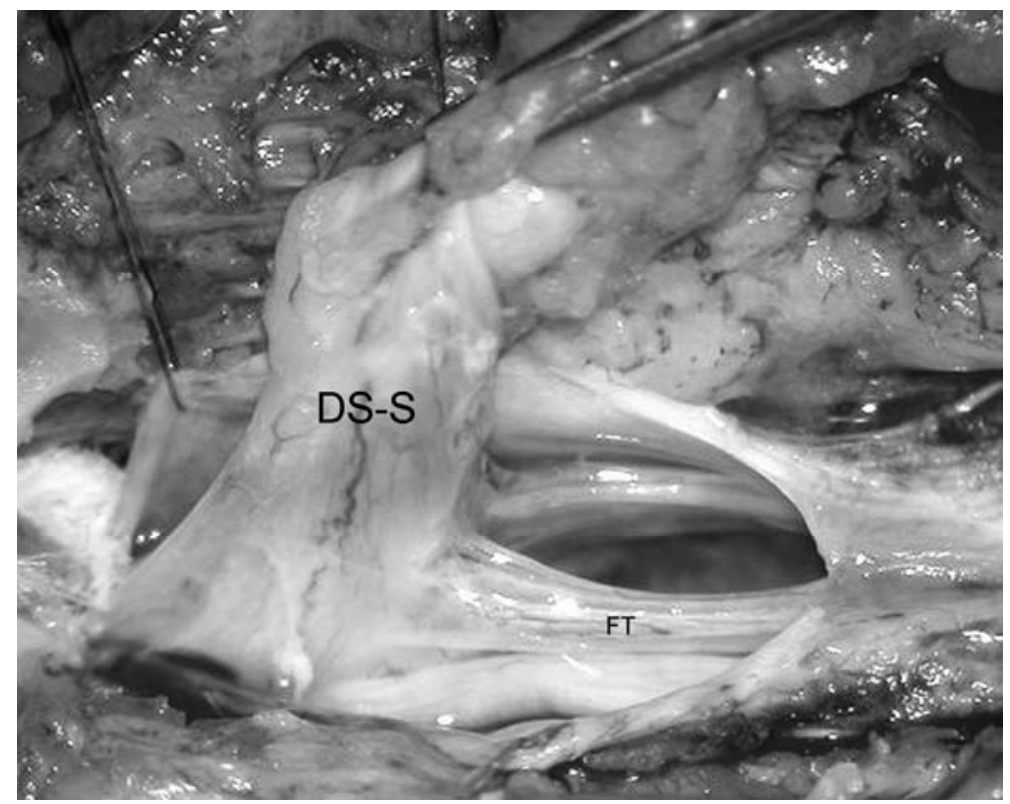

Figure 4.2 Intraoperative picture (case 1), showing the dermal sinus-like stalk (DS-S) being attached to the conus medullaris. FT = filum terminale.

See page 155 for colour figure.

\section{Outcome}

The child recovered well after surgery and remained neurologically intact. Histological examination (Figure 4.3) of the stalk revealed connective tissue and fat, with nervous elements and muscle tissue visible in some parts of the resected specimen. The absence of a lumen was confirmed. 


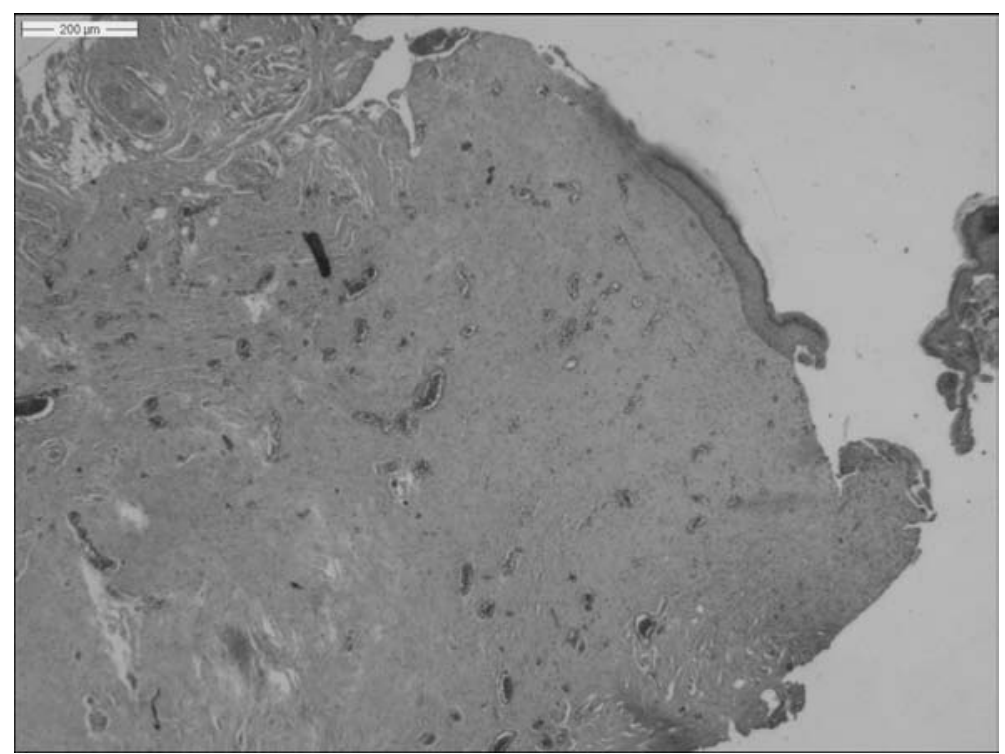

Figure 4.3 Photomicrograph of the resected stalk in case 1. Most of it is composed of connective tissue.

See page 156 for colour figure.

\section{Case 2}

\section{Clinical condition}

This patient presented at birth with a lumbar dimple, surrounded by some hypertrichosis. At that time no neurological deficits were noted. At the age of seven years she was referred to our hospital with pain in the lower extremities. Clinical examination revealed a lumbar dimple, without orifice, and a pes cavus, while urodynamic evaluation showed a mild detrusor instability.

\section{Imaging}

At MR imaging the conus was estimated at L1-L2. At L2-L3 level a tenting of the dura was seen, presumably continuing as a trajectory up to the skin defect (Figure 4.4). 


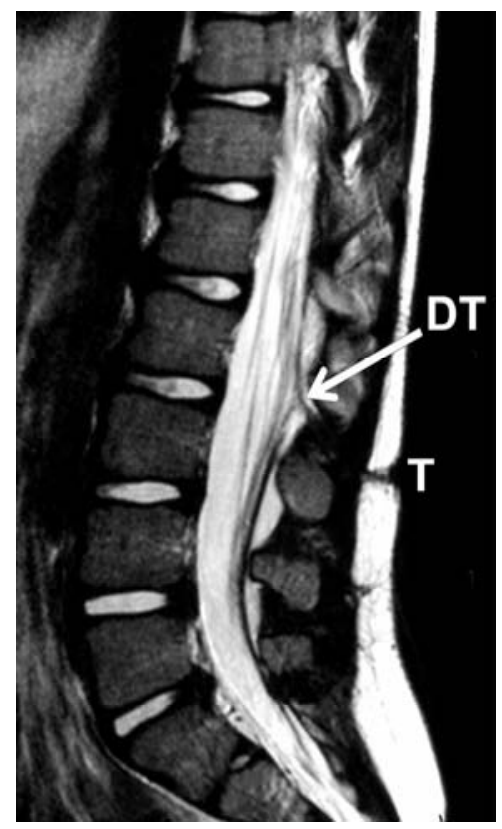

Figure 4.4 Sagittal T2-weighted MR image showing the subcutaneous trajectory $(T)$ as well as the tenting of the dural sac (DT).

\section{Surgery}

After circumscribing the dimple, a fibrous stalk was found continuous with the skin, passing the subcutaneous and muscular layers up to the dura. The stalk was seen traversing the dura, being loosely attached to the arachnoidal membranes. At that point it was dissected from the arachnoidal membranes and resected en bloc. No lumen could be detected in the stalk at intraoperative inspection.

No infected areas or dermoid masses were found. Because a tight filum terminale was noted, it was sectioned.

\section{Outcome}

The postoperative course was uneventful and the child reported a significant decrease of pain in the lower extremities.

Histological examination of the resected stalk revealed connective tissue, of which some parts were positively stained with epithelial membrane antigen (EMA), indicating a dural origin. A considerable amount of fat and large vessels 
were seen. The absence of a lumen in the resected stalk was confirmed (Figures 4.5A/4.5B).
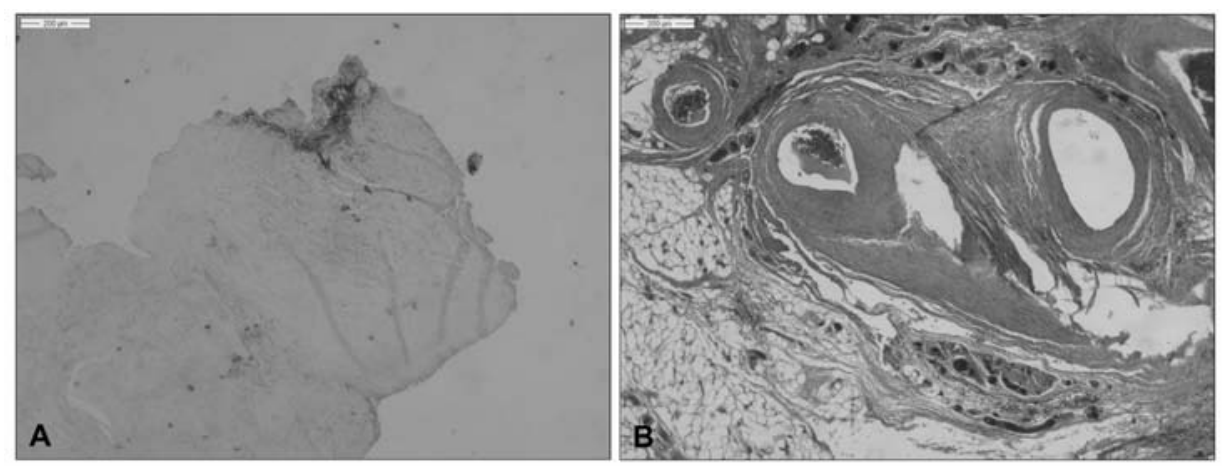

Figure 4.5 Photomicrographs of the resected stalk in case 2. Some areas are EMA positive (A). Note the large vessels and a considerable amount of fat (B).

See page 156 for colour figure.

\section{Case 3}

\section{Clinical condition}

At birth this child presented with two dimples, located at lumbosacral and coccygeal levels. Hypertrichosis was noted around the lumbosacral dimple (Figure 4.6). No neurological deficits were found at clinical examination.

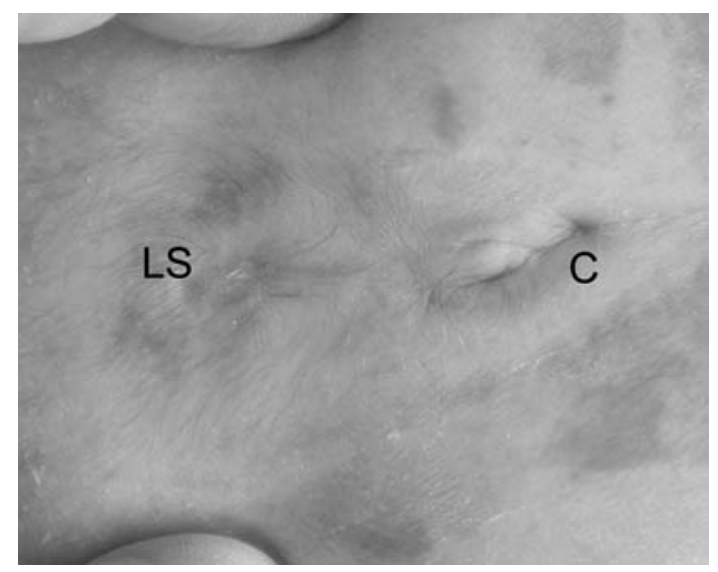

Figure 4.6 Preoperative picture of dimples in case 3. C = coccygeal, LS = lumbosacral. See page 156 for colour figure. 


\section{Imaging}

MR imaging showed a tethered cord. The level of the conus was estimated at L4. A trajectory was seen connecting the upper skin defect with the intradural space.

\section{Surgery}

Both dimples were surgically explored. The coccygeal dimple ended subcutaneously.

A solid tract was found underneath the lumbosacral dimple, continuing up to the conus medullaris at the L5 level. Although no dermoid or epidermoid tissue was found, a lipomatous mass at the level of connection with the conus was seen. The tract was amputated from the conus distal to the level at which the lowest roots were seen branching off.

\section{Outcome}

The child recovered well after surgery and remained neurologically intact. Histological examination of the resected stalk revealed connective tissue and large areas of fat. In some parts neural tissue was noted (S100+). The absence of a lumen in the stalk was confirmed (Figure 4.7).

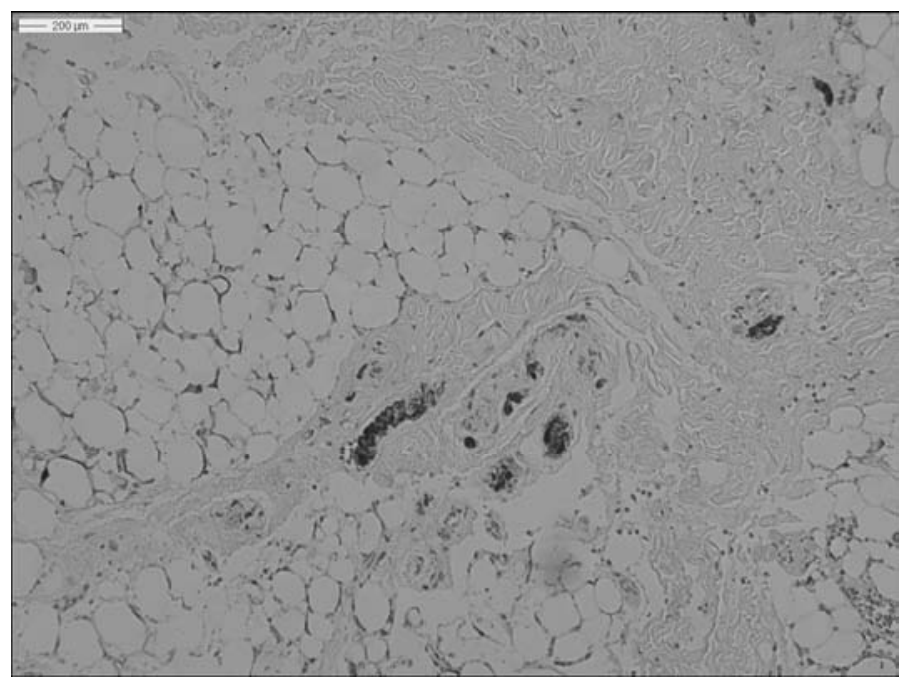

Figure 4.7 Photomicrograph of the resected stalk in case 3. Note the large fat cells and the small parts, which are positively stained with S100. (H\&E, S100 staining). See page 157 for colour figure. 


\section{Discussion}

The authors present three cases in which the diagnosis of a dermal sinus (DS) was initially suggested. Although a dimple with an underlying tract continuing to the intradural space was found in all cases, the clinical and anatomical findings did not resemble that of a DS (Table 4.1, Figure 4.8).

All cases presented with a dimple without orifice. Neither a lumen nor a dermoid-epidermoid tumor was detected in any of the resected specimens. None of these patients presented with signs of infection. These findings do not fit in the clinical picture of a typical DS, which presents with an orifice at the skin surface, from which an epithelial-lined fistula travels inward, up to the spinal cord.

Table 4.1 Characteristics of the dernal sinus and dermal-sinus-like stalk.

\begin{tabular}{lll}
\hline & Dermal sinus & Dermal-sinus-like stalk \\
\hline Skin defect & Open & Covered \\
Stalk contains a lumen & Yes & No \\
Histological findings of stalk & Lined with stratified squamous & Connective tissue, fat, muscle; nervous \\
& epithelium & structures-dural elements might be found \\
Meningitis & Yes & No \\
Empyema & Yes & No \\
Dermoid-epidermoid tumor & Yes & No \\
Dural sleeve & Directed towards spinal cord & Directed towards skin \\
\hline
\end{tabular}

\section{Etiology of the spinal congenital dermal sinus}

The embryological pathogenesis of a DS is not known. It has been suggested that the separation between surface ectoderm and neural ectoderm did not take place during embryonic development, resulting in an epidermal-neural fistula ${ }^{9,11,14,18 .}$.

In the normal human embryo, closure of the neural tube takes place in the fourth developmental week. Subsequently, the neural ectoderm is separated from the surface ectoderm. The former gives rise to the brain and spinal cord, the latter to the epidermis. The mesoderm then develops in between these two layers of ectoderm, giving rise to the spinal arches, paraspinal muscles, dura and other mesodermal derivatives.

In case of a DS, the tract leads from epidermis for a variable distance through dermis, subcutaneous fat, fascia, muscle, vertebral arch and meninges up to the spinal cord. Of these layers, only the epidermis and the spinal cord are of ectodermal origin. Histological analysis of a typical DS tract, reveals that this fistula has a lumen, which is lined by stratified squamous epithelium immediately surrounded by dermal tissue ${ }^{19}$. This epithelium resembles normal epidermis. 
From this point of view, it is likely that a non-disjunction of both ectodermal layers gives rise to a persistent epidermal-neural fistula.

What happens after this event, that causes the two layers of ectoderm not to detach?

The hollow fistula that comes to development might be the result of the cutaneous ectoderm being carried down ventrally, into the future spinal canal as it is attached to the neural ectoderm. Within this epithelium-lined invagination, normal epidermal appendages come to development. This would explain the presence of hairs and sebaceous glands in the inclusion tumors, frequently found in case of a DS.

We previously described a typical configuration of the dura at the point where the dermal sinus tract enters the intradural space. A dural sleeve is always seen, directed from the dural sac into the intradural space ${ }^{17}$. The direction of this mesodermal sleeve could be determined by a process in which the meninx primitiva is dragged into the future dural sac together with the cutaneous ectoderm.

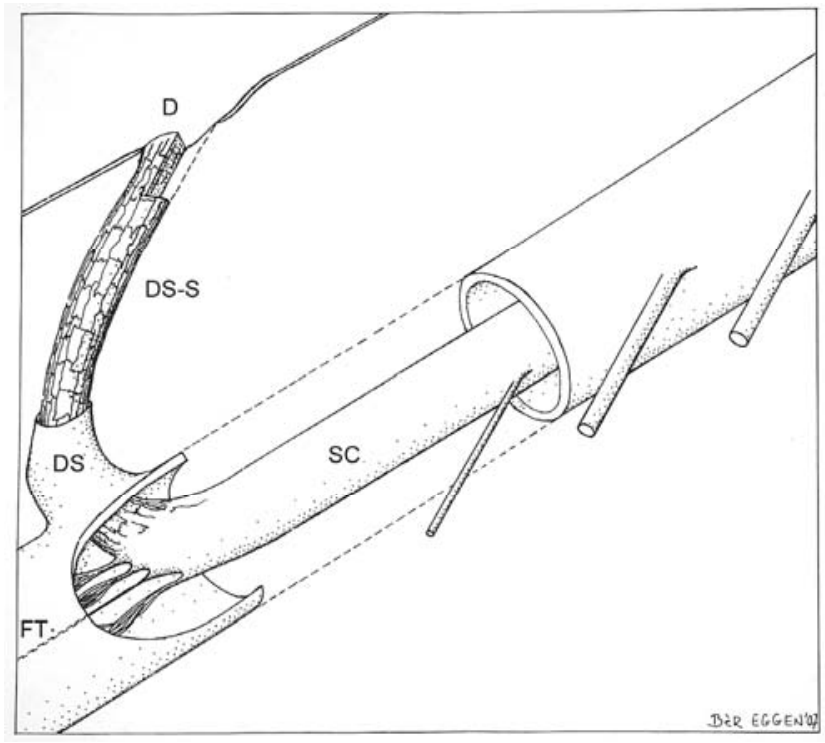

Figure 4.8 Artist illustration of the anatomical configuration of the dermal sinus-like stalk. $\mathrm{D}=$ dimple, $\mathrm{DS}-\mathrm{S}=$ dermal sinus-like stalk, $\mathrm{DS}=$ dural sleeve, $\mathrm{FT}=$ filum terminale, $\mathrm{SC}=$ spinal cord . 


\section{Etiology of the spinal dermal sinus-like stalk}

To the best of our knowledge, DS-like stalks, as presented in this report, have never been described in the literature as a significantly different type of the DS. The fibrous stalk may represent a particular appearance of a dermal sinus tract. We suggest that in such cases the inner epidermal lining of the hollow tract has degenerated, and the canal has been obliterated.

However, the DS-like stalk may also have a different etiology. The absence of a central lumen may indicate that the model of inward dragging of epidermis does not fit. The absence of an open skin defect and of intramedullary epidermoid or dermoid masses may indicate a lack of ectodermal involvement in the etiology of the DS-like stalk.

Since the tracts are composed of fibrous tissue of presumed mesodermal origin, after separation of epidermal and neural tissue, invading mesodermal cells may have formed a tight and persistent connection between the two.

In two cases dorsal tenting of the dura was found. In case 1 this was noted peroperatively as in case 2 it was clearly shown by MR imaging. Histological examination of the resected tract in case 2 showed tissue with dural origin. This was previously described by Scotti et al., who reported on a case of a thoracic DS with dorsal tenting of the dura. Although histological examination of this tract revealed an epidermal-lined tract, it was mainly composed of dense collagenous tissue with foci of meningeal tissue ${ }^{15}$.

Additionally, this dorsal tenting was previously described by Aydin et al. ${ }^{2}$ in a case of a thoracocervical dermal sinus. In this report multiple vertebral body anomalies were noted, suggesting additional mesodermal developmental failure.

If the primary failure in case of a DS-like stalk is the same as the one presumed in case of a DS, a non-disjunction of neural and cutaneous ectoderm takes place. In case of a DS, the epidermis is dragged into the future spinal canal along with the neural ectoderm. However, in the cases described in this report, mesenchymal and neural elements were detected in the stalks. Thus in case of a DS-like stalk this 'dragging' may take place in an opposite (ventral-dorsal) direction in which the neural ectoderm and future dura mater are pulled out of the future spinal canal, producing a stalk representing an atrophic mesodermalneural stalk rather than a fistula composed of epidermal tissue.

\section{Imaging in case of a dermal sinus-like stalk}

MR imaging is imperative in case of a dermal sinus or dermal sinus like stalk $^{4,6,16}$, but interpretation can be difficult ${ }^{3,20}$. In case of a lumbosacral tract, the low position of the tethered conus medullaris can be depicted.

In the three cases described in this report, MR imaging vaguely demonstrated the trajectory of the stalk. However, the connection of the stalk with the spinal 
cord was not demonstrated in any case. No empyema or epidermoid-dermoid mass were seen, whereas these are common findings in case of a typical $\mathrm{DS}^{3,4,17}$. In case 2 , dorsal tenting of the dura was clearly demonstrated.

\section{Indications for surgery}

Of three patients, one presented with mild neurological deficits. Although the other two patients did not have any symptoms, all three underwent surgical exploration because a DS was feared.

Theoretically, there is no need for urgent surgical exploration in case of a DSlike stalk because there is no open connection between the skin surface and the intradural space which minimizes the risk of intraspinal infection.

However, differentiating a true DS from a DS-like stalk after clinical and radiological evaluation is difficult. Therefore, the authors recommend urgent surgical exploration in all cases. Moreover, the connection of the stalk with the spinal cord can give rise to a tethering mechanism. In addition a tight filum terminale can be found, tethering the cord.

During surgical exploration, an untethering should be performed simultaneously.

\section{Conclusion}

Whenever a patient presents with a skin-covered dimple, cranial to the gluteal cleft, without signs of infection and without radiological signs of abscess or intraspinal dermoid-epidermoid tumor, a dermal sinus-like stalk should be suspected. Even though this malformation is not associated with intraspinal infection, it is difficult to distinguish a true DS from a DS-like stalk based on clinical and MR imaging. Therefore, we recommend surgical exploration and careful histopathological examination of the tract in all cases. 


\section{References}

1. Ackerman LL, Menezes AH. Spinal congenital dermal sinuses: a 30-year experience. Pediatrics 2004;112:641-647

2. Aydin K, Sencer S, Minareci O. Thoracocervical dorsal dermal sinus associated with multiple vertebral body anomalies. Neuroradiology 2001;43:1084-1086

3. Barkovich AJ, Edwards M, Cogen PH. MR evaluation of spinal dermal sinus tracts in children. AJNR Am J Neuroradiol 1991;12:123-129

4. Bruff $P$, Sgouros S. Lumbar dermoid cyst causing pyomyelia in a child. Pediatr Neurosurg 2002;36:162-163

5. Candon E, Frerebeau P. [Bacterial abscesses of the spinal cord. Review of the literature (73 cases)]. Rev Neurol (Paris) 1994;150:370-376

6. Dev R, Husain M, Gupta A, Gupta RK. MR of multiple intraspinal abscesses associated with congenital dermal sinus. AJNR Am J Neuroradiol 1997;18:742-743

7. DiTullio MV Jr. Intramedullary spinal abscess: a case report with a review of 53 previously described cases. Surg Neurol 1977;7:351-354

8. el-Gindi S, Fairburn B. Intramedullary spinal abscess as a complication of a congenital dermal sinus. Case report. J Neurosurg 1969;30:494-497

9. French BN. The embryology of spinal dysraphism. Clin Neurosurg 1983;30:295-340

10. James CCM, Lassman LP: Spinal Dysraphism. London: Butterworth \& Co, 1972

11. Kanev PM, Park TS. Dermoids and dermal sinus tracts of the spine. Neurosurg Clin N Am 1995;6:359-366

12. Maurice-Williams RS, Pamphilon D, Coakham HB. Intramedullary abscess--a rare complication of spinal dysraphism. J Neurol Neurosurg Psychiatry 1980;43:1045-1048

13. Morandi X, Mercier P, Fournier HD, Brassier G. Dermal sinus and intramedullary spinal cord abscess. Report of two cases and review of the literature. Childs Nerv Syst 1999;15:202-206; discussion 207-208

14. Ripley $\mathbf{W}$, Thompson DC. Pilonidal sinus as a route of infection in a case of staphylococcus meningitis. Amer J Dis Child 1928;36:785-788

15. Scotti G, Harwood-Nash DC, Hoffman HJ. Congenital thoracic dermal sinus: diagnosis by computer assisted metrizamide myelography. J Comput Assist Tomogr 1980;4:675-677

16. Shah RK, Chaljub G, Swischuk LE. Lower cervical dermal sinus tract and associated intraspinal abscess causing meningitis in a child. Emerg Radiol 2003;10:160-162

17. van Aalst J, Beuls EA, Cornips EM, Vanormelingen L, Vandersteen M, Weber JW, Vles JS. Anatomy and surgery of the infected dermal sinus of the lower spine. Childs Nerv Syst 2006;22:1307-1315

18. Walker E, Bucy PC. Congenital dermal sinuses: a source of spinal meningeal infection and subdural abscesses. Brain 1934;57:401-421

19. Weprin BE, Oakes WJ. Coccygeal pits. Pediatrics 2000;105:E69

20. Yang BP, McLone DG. Blood vessel mimicking a dermal sinus tract. Pediatr Neurosurg 2004;40:88-89 


\section{Spinal congenital dermal sinus in a chick embryo model Laboratory investigation}

Jasper van Aalst, Toon F.M. Boselie, Emile A.M. Beuls, Johan S.H. Vles, Henny W.M. van Straaten

Journal of Neurosurgery: Pediatrics 2009;3:24-28 


\section{Abstract}

\section{Object}

The origin of spinal congenital dermal sinuses is not known. A local nondisjunction of the closing neural tube and the epidermal ectoderm is thought to be the cause of this malformation. In this experimental study, a nondisjunction was mimicked in the chick embryo to create an animal model for the dermal sinus.

\section{Methods}

A piece of amniotic tissue was implanted in the closing neural tube in ovo in chick embryos at two days of incubation. A total of 50 embryos were manipulated. After a further incubation time of two to seven days, the embryos were macroscopically and histologically evaluated.

\section{Results}

Dermal sinus-like anomalies were induced in 24 embryos. The induced abnormalities varied from superficial, epidermal lesions to epidermal dimples continuing as a strand of tissue toward the neural tube. This strand invariably was of non-neuronal origin. Additionally, in three embryos a split cord malformation was noted, most likely caused by damage to the neural tube during implantation.

\section{Conclusions}

Implantation of donor amniotic tissue in the closing chick neural tube does result in a dimple, from which a strand of tissue continues to the neural tube in various cases, indicating that formation of a dermal sinus-like anomaly can be successfully induced by experimental continuation of the connection between neural tube and surface ectoderm. This finding strengthens the hypothesis that a human DS arises after nondisjunction of neural tube and surface ectoderm. 


\section{Introduction}

A spinal congenital dermal sinus is a developmental anomaly presenting with a cutaneous defect along the midline of the spinal axis. From the skin surface, an epithelium-lined fistula develops, continuing for a variable distance through the dermis, subcutaneous fat, fascia, muscle, the vertebral arch and meninges. In most cases, it continues intradurally or even into the spinal $\operatorname{cord}^{6}$. The incidence of this rare malformation is estimated as 1 per 2500 live births ${ }^{8}$.

Although the clinical presentation of patients with dermal sinuses varies, the majority presents with a life-threatening intradural infection ${ }^{6,17}$. Therefore, clinical perinatal examination should always include a thorough examination of the entire midline axis. In case of a dermal sinus, treatment consists of early surgical exploration with resection of the entire fistulous tract.

The origin of a dermal sinus is not known. In the literature, it has been hypothesized that it might be the result of a non-disjunction of surface ectoderm and neural ectoderm during final closure of the neural tube $e^{6,13,19}$. However, to the best of our knowledge, this hypothesis has never been studied in an experimental animal model.

In the human embryo, closure of the neural tube takes place in the 4th developmental week. Initially, the neural plate begins developing in the midline ectoderm. During neurulation, the lateral walls and folds of the neural plate bend dorsally and subsequently medially. The bilateral transition zones of surface ectoderm and the neural plate make contact in the midline, forming a continuous sheet of surface ectoderm and a closed tube of neural ectoderm. Subsequently, the neural tube and surface ectoderm detach from each other and mesoderm starts to fill the space between them. The surface ectoderm then develops into the epidermis, and the neural tube gives rise to the brain and spinal cord ${ }^{3}$.

A fistulous tract of a dermal sinus thus may arise if there is a local nondisjunction between surface ectoderm and the neural tube, perhaps due to a local nonclosure of both tissue layers followed by a nondisjunction of both structures, or due to the latter only. The locally persistent connection between both layers becomes elongated more and more by intervening mesoderm, resulting in a tract.

A dermal sinus has incidentally been reported in the rat and mouse, and frequently in several breeds of dogs, most importantly the Rhodesian ridgeback ${ }^{1,2,4,7,10}$. In the case of the latter, a dominant genetic mutation was recently identified ${ }^{15}$. However, no experimental animal model exists for this condition.

In this report, we describe the results of an effort to mimic the process of nondisjunction and to create an animal model for the dermal sinus. We hypothesized that local failure of the neural tube to close causes a local nondisjunction. To test this hypothesis, the recently approximated region of the 
neural tube (a region where the neural tube walls have approached each other and just begin to adhere) of chick embryos at two days of incubation was reopened locally, after which a piece of amniotic membrane from a donor chick embryo was implanted. This material was supposed to prevent the normally occurring reclosure of an experimentally reopened neural tube, thereby preventing disjunction of neural tube and ectoderm. The decision to use amnion was based on the assumption that amnion is a relatively inert tissue. In every human embryo it is present as a thin layer, covering the neural plate. Moreover, because amnion has a high level of differentiation it was thought to act only as an implant and the chances of proliferation of the amnion itself would be low.

\section{Methods}

Fertilized eggs of White Leghorn chickens (Gallus gallus) were incubated in a commercially available incubator at $39^{\circ} \mathrm{C}$ and an air humidity of approximately $55 \%$. Donor embryos were incubated for a period of $115 \pm 23$ hours, and acceptor embryos were incubated for $52 \pm 7$ hours.

A small piece of donor amniotic membrane was dissected in ovo, using iridectomy scissors, and stored in Locke fluid until implantation. To manipulate the acceptor embryo in ovo, the eggshell was windowed and the embryo was lifted up for better viewing and manipulation by applying Locke fluid. After incising the vitelline membrane, a recently approximated part of the neural tube was reopened over a length of about $100 \mu \mathrm{m}$ using an electrolytically sharpened tungsten needle. In a recently approximated part of the neural tube, the neural folds are loosely adhered only, allowing reopening rather easily ${ }^{18}$. A small piece of amniotic tissue was then manoeuvred between the opened tube walls (Figure 5.1).

The embryos had a mean of $14 \pm 5$ somites at the moment of implantation, and the level chosen for implantation was $13 \pm 5$ (that is, one level cranial to the last formed somite).

After manipulation the embryo was lowered into the egg, the shell window was closed with adhesive tape, and the egg was replaced in the incubator. The postmanipulation incubation time ranged from two to seven days. The embryos were subsequently removed from the egg, fixated in Bodian fixative, dehydrated, embedded in paraffin, transversely sectioned at $5 \mu \mathrm{m}$ and stained with Haematoxylin-Eosin. Selected sections were immunostained for neural cell adhesion molecule (chick-NCAM), as a marker for cells of neural origin ${ }^{11,14}$. The mouse anti-NCAM monoclonal antibody developed by Rutishauser was obtained from the Developmental Studies Hybridoma Bank developed under the auspices of the National Institute of Child Health and Human Development and maintained by The University of lowa, Department of Biological Sciences. 


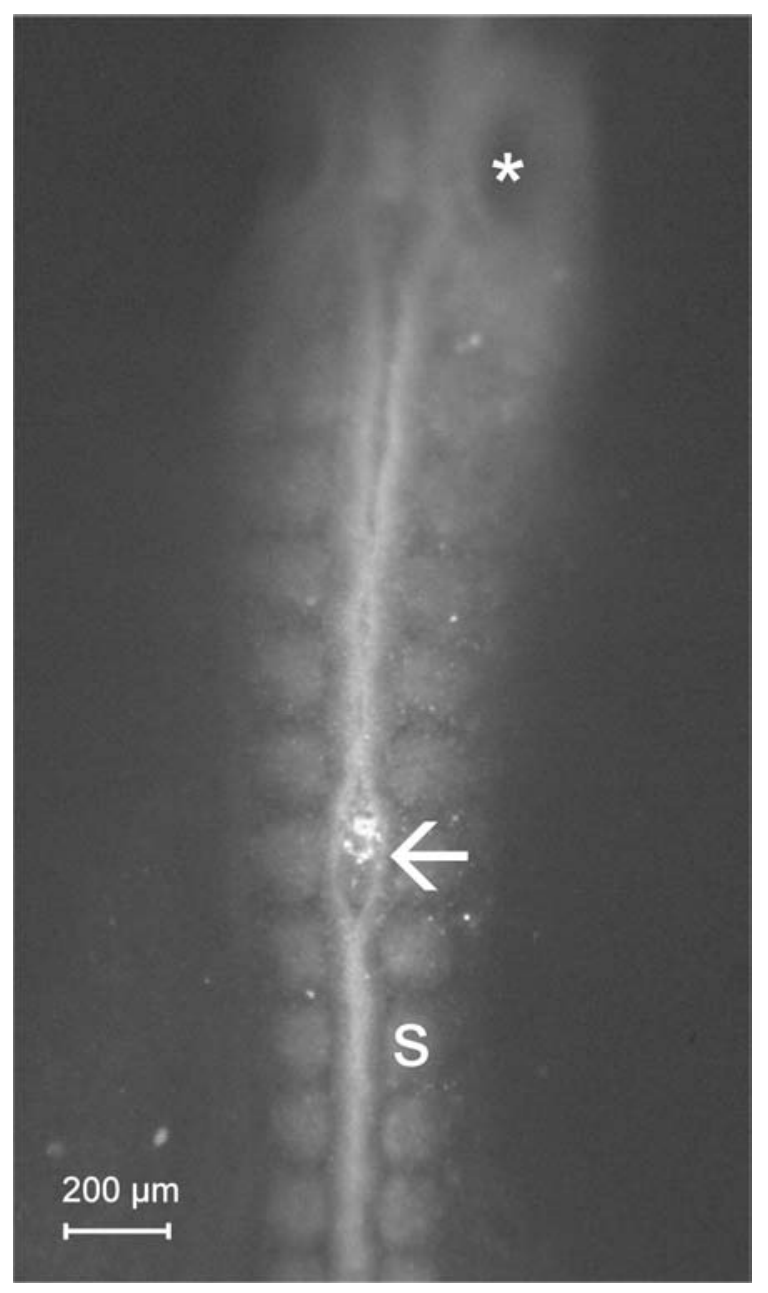

Figure 5.1 Intraoperative view. Arrow points at implant in the reopened neural tube. ${ }^{*}=$ otic vesicle, $\mathrm{S}=$ somite. 


\section{Results}

Fifty embryos were manipulated. At the time of analysis, 12 embryos $(24 \%)$ were dead, and partially resorbed, and could therefore not be evaluated. The 38 embryos $(76 \%)$ that survived the manipulation and subsequent incubation were further analyzed. The embryos were divided into the following three groups based on the malformations that had developed:

1. Embryos without abnormalities

2. Embryos with superficial epidermal lesions

3. Embryos in which an epidermal lesion was present, from which a tract was seen, continuing toward the neural tube.

\section{Group 1: No abnormalities}

Fourteen embryos (37\%) appeared normal upon careful evaluation, both macroscopically and stereomicroscopically. If there was any doubt whether an abnormality was present, the embryo was histotechnically processed and microscopically evaluated; this procedure was performed for seven embryos. None of these showed abnormalities.

\section{Group 2: Embryos with superficial lesions}

In six embryos (16\%), only superficial epidermal lesions were seen. The level of manipulation was recognized by indentation or protrusion of the epidermis, or by marked lordosis of the spinal axis. An example of this type of lesion can be seen in Figure 5.2 .

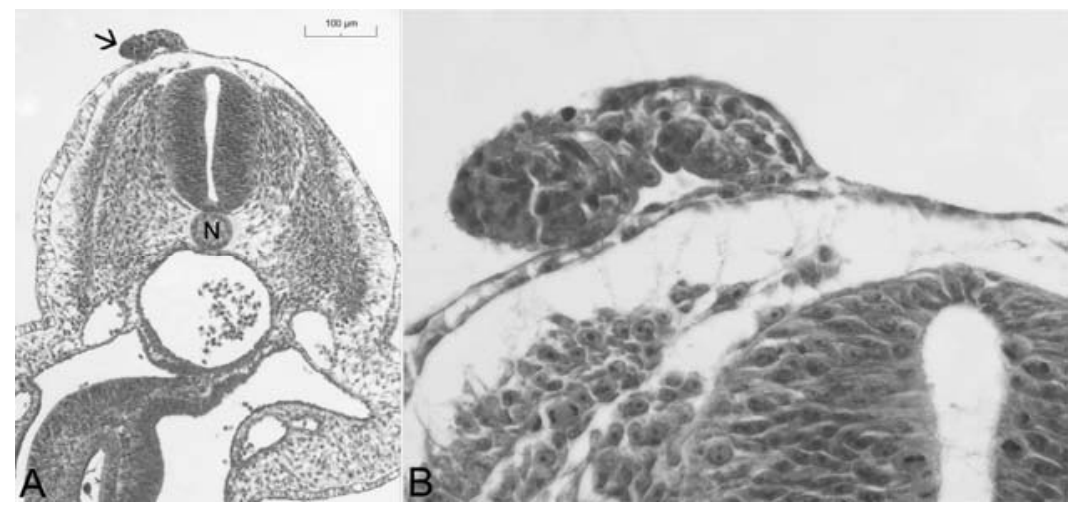

Figure 5.2 Photomicrographs. A: A tract of tissue (arrow) protrudes from the epidermis. $\mathrm{N}=$ notochord. B: Enlargement of Figure 5.2A. The pink tissue inside the protrusion has the appearance of amnion. H\&E staining, original magnification x 1250 . See page 157 for colour figure. 
Some of the superficial lesions consisted of taillike protrusions of the epidermis, often encapsulating some tissue, which was identified as remnants of the implanted amniotic membrane. Other lesions were smaller, being no more then small lumps of the same intermingled epidermis and amniotic membrane, or a local thickening of the epidermis. The underlying mesodermal tissue and neural tube were normal in these embryos. Staining for NCAM in one of the embryos showed staining of neural tube, notochord and myotomes, but not of the epidermal malformation.

\section{Group 3: Dermal sinus-like tract}

In 18 embryos (47\%), 19 lesions were found in which a dermal sinus-like tract was seen. One of these embryos had been manipulated at two separate levels, and a dermal sinus-like tract was noted at both levels. The abnormalities were only present at the manipulated level, and absent at levels caudal and cranial to it, except for one case, in which an additional dysraphic condition was seen, starting at the manipulated level and continuing caudally. In three of the embryos within this group, a split cord malformation was noted, at the same level as the DS-like tract (Figure 5.3).

The neural tube was closed in all cases, with the deepest infiltrating tracts touching the roof plate of the neural tube, or the dorsal aspect of the spinal cord in the older embryos.

Although the lesions found in this group showed many similarities, a variable anatomical configuration was seen.

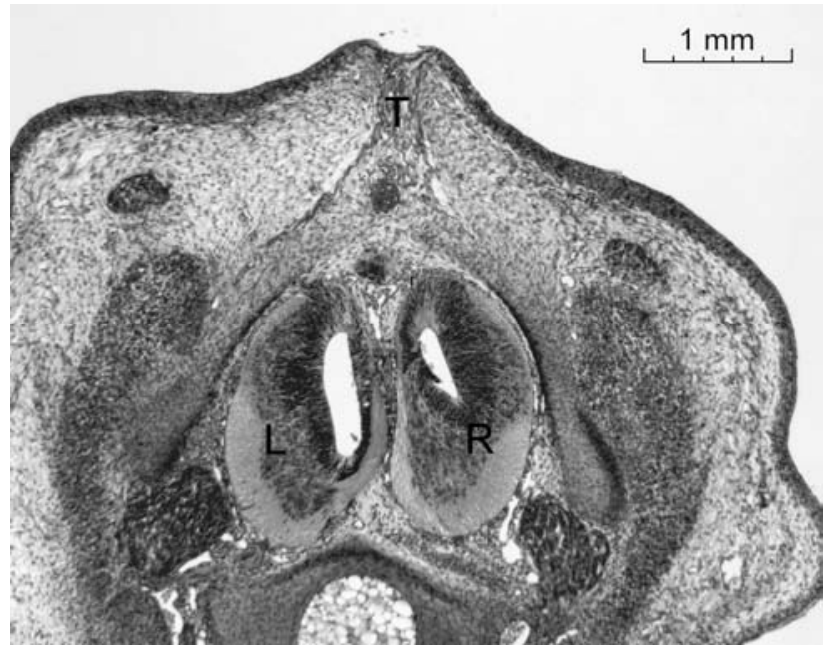

Figure 5.3 Photomicrograph. HE staining. Example of a split cord malformation as was seen in some of the embryos. $T=$ tract, $\mathrm{L}$ and $\mathrm{R}=$ left and right hemicord.

See page 158 for colour figure. 
Two main types of tracts were noted; cystic lesions and strands of fibrotic tissue. In several cases these were found together, in the form of a cystic lesion close to the neural tube or spinal cord, with strands of fibrotic tissue reaching up to the epidermis (Figure 5.4). These strands resembled the combination of epidermis and amniotic membrane found in the superficial lesions described in group 2, but now with an elongated aspect in dorsoventral direction, and signs of fibrosis, especially in the older embryos. At the superficial, epidermal end of the tracts a varying degree of dimple formation was found, suggesting traction in a dorsoventral direction.

Two of these cases were stained for NCAM. They showed staining of the neural tube, but not of the artefact (Figure 5.4).

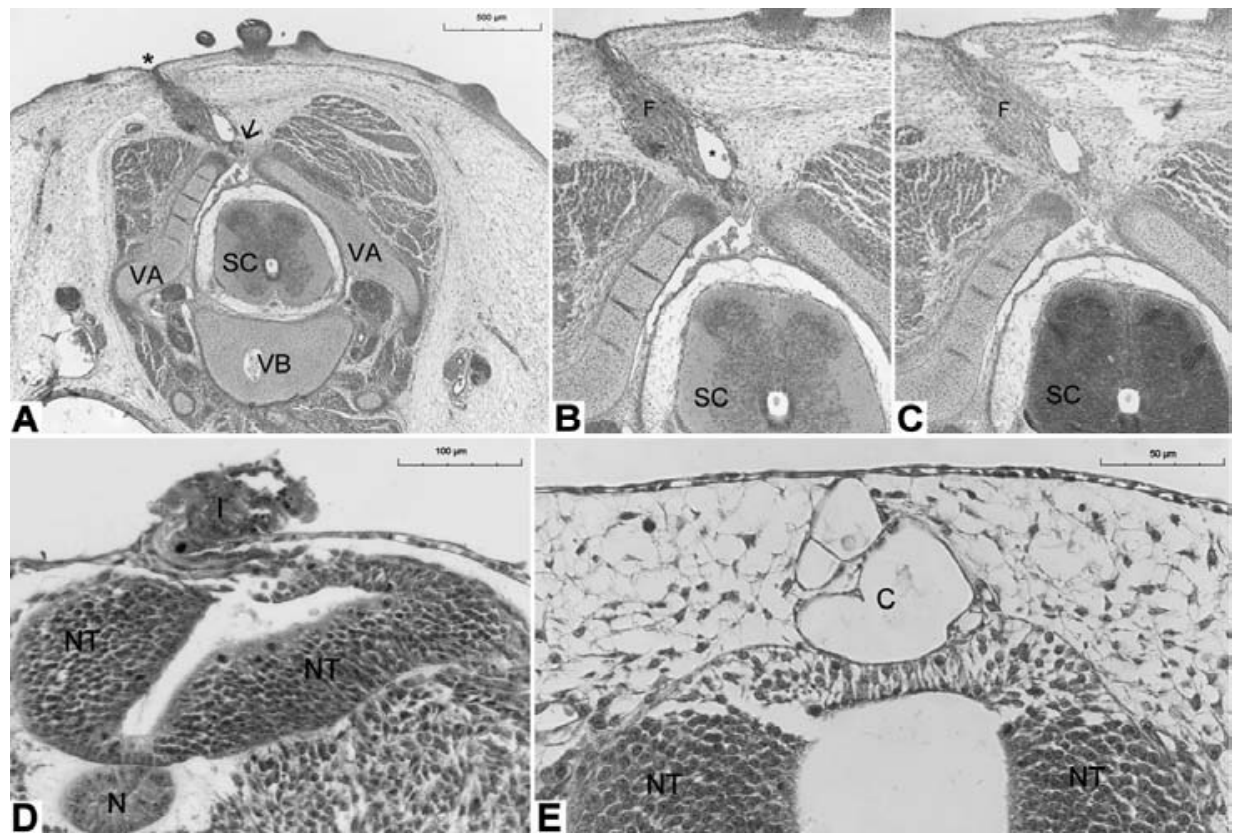

Figure 5.4 Photomicrographs. A: A section obtained in a 9-day embryo. An epidermal dimple (indicated by the asterisk) is present, attached to a tract, which continues through a closure defect (arrow) in the vertebral arch (VA). The vertebral body (VB) and spinal cord (SC) have a normal appearance. B. Enlargement of panel A. Note the vacuole (asterisk) within the fibrous tract (F). C. Staining of the spinal cord (SC) but not of the fibrous tract. D. Three-day embryo; the implant (I) seems to infiltrate in the disturbed neural tube (NT). The notochord $(\mathrm{N})$ is seen underlying the neural tube. $\mathrm{E}$. Four-day embryo; cystic tract (C), which makes contact with the neural tube. $\mathrm{H}$ \& $\mathrm{E}$ $(A, B, D$, and $E)$; NCAM (C); original magnification $\times 85$ (B and C).

See page 158 for colour figure. 


\section{Discussion}

To date, the etiology of the spinal dermal sinus is not known. One of the first descriptions of a spinal congenital dermal sinus was published in $1887^{5}$. In the early $20^{\text {th }}$ century, more reports were added in which the difference between the true DS and the more common sacrococcygeal dimple was emphasised. It is from this period that theories on the etiology of the DS have its origin.

In 1926, Moise described a case of a lumbosacral DS with associated meningitis ${ }^{9}$. He concluded from this case that a pilonidal sinus was present in combination with spina bifida and that this malformation probably had a different etiology compared to a 'simple' pilonidal sinus. Moise suggested that obliteration of the medullary canal was disturbed, giving rise to a direct connection between skin and spinal cord.

Walker and Bucy proposed in 1934 that the DS is a result of a nondisjunction, an incomplete separation between cutaneous ectoderm and neural tube. The neural tube would subsequently drag a narrow invagination of the overlying skin down with it, clothing it with a mesodermal covering ${ }^{19}$. Although this theory is generally accepted in current literature, it was never tested in an experimental setting.

In this paper, we have described the results of an experiment in which a dermal sinus-like malformation was produced experimentally in the chick embryo, by implanting a piece of rather inert material, amnion, in the closing neural tube. To the best of our knowledge, this is the first report demonstrating such a tract after a local non-disjunction was mimicked in an experimental animal model.

The malformations that developed showed several similarities with the dermal sinuses as described in humans: a dimple or tract extending inwards from the skin, frequently continuing to the spinal cord. The induced abnormalities varied from superficial epidermal lesions to tracts continuing for a variable depth through the underlying mesodermal tissue. The abnormal tissue that was noted in these tracts, appeared to consist of remnants of the implanted amnion, intermingled with fibrotic tissue. In many cases the tract continued to the neural tube, which was closed in all cases. The tract did not in any case continue inside the neural tube. Cystic lesions in some of the tracts suggested a hollow fistula, as is commonly seen in the human dermal sinus. However, after evaluation of consecutive sections in several embryos, such a fistula could not be demonstrated.

Staining of the tract for NCAM was negative, indicating a non-neural origin.

The results of our experiments support the hypothesis that a spinal DS might result from an ectodermal nondisjunction. Although the cutaneous ectoderm is known to separate from the neural ectoderm after the neural tube has closed, the factors leading to this disjunction are poorly understood. Apoptosis seems 
to be of importance in this complex process, as was described by Selcuki et al. $^{16}$.

We hypothesized that the nondisjunction in the embryos described in our experiments was caused by a local reopening of the very recently closed neural tube and implantation of a piece of donor amniotic membrane, thereby preventing definitive closure of the neural tube and subsequent disjunction at that level.

During further development, the neural tube must have worked the amnion out of its cavity, possibly by the pressure of the cerebrospinal fluid, which is necessary for normal brain development. The tube subsequently closed, but the amnion and the epidermal ectoderm remained attached to the tube. When the mesoderm subsequently interspersed between neural tube and epidermis, the tract must have elongated, pulling the epidermal ectoderm gradually inward.

Another explanation for the development of the tracts could be that the implanted amniotic tissue did not induce a nondisjunction, but instead was pushed out of the neural tube and subsequently encapsulated in the developing mesoderm. According to this hypothesis, the tracts might represent a mesodermal sheath around the amniotic implant.

In some cases only a superficial malformation with the appearance of a human proboscis developed, possibly due to the fact that the implanted tissue was more adherent to the surface ectoderm than to the neural tube. Subsequently, during embryonic development, the implant could then have been lifted upward, out of the neural tube, together with the epidermal ectoderm only. A second reason for the development of such superficial malformations could again be the pressure of the cerebrospinal fluid, which may have flushed out the implanted piece of tissue immediately after implantation.

The split cord malformation, as was seen in three embryos, was possibly caused by incising the neural tube too deep, through both roof plate and floor plate, thereby dividing it into two hemicords. This resembles the method from which Rilliet et al. developed a model for diastematomyelia, although in that experiment the underlying notochord was incised as well ${ }^{12}$. 


\section{Conclusion}

It can be concluded from the results of our experiments that an experimentally induced adhesion between surface ectoderm and neural tube resembles a nondisjunction, suggesting an important role for this mechanism in the etiology of the spinal dermal sinus.

In case of the human spinal dermal sinus, this nondisjunction may possibly be caused by material floating in the amniotic cavity, the amnion itself, or apoptotic cells from the neural tube, interfering with the process of closure of the neural tube at a very local level, which is followed then by a nondisjunction. Additionally, as was demonstrated in the Rhodesian ridgeback, a genetic failure giving rise to the human dermal sinus is not unlikely. More research on the mechanisms of disjunction and the pathological nondisjunction is needed to further elucidate the etiology of the spinal dermal sinus. 


\section{References}

1. Anderson DM, White RA. Nasal dermoid sinus cysts in the dog. Vet Surg 2002;31:303-308.

2. Booth MJ. Atypical dermoid sinus in a chow chow dog. J S Afr Vet Assoc 1998;69:102-104.

3. Copp AJ, Greene ND, Murdoch JN: The genetic basis of mammalian neurulation. Nat Rev Genet 2003;4:784-793.

4. Cornegliani L, Jommi E, Vercelli A. Dermoid sinus in a golden retriever. J Small Anim Pract 2001;42:514-516.

5. Dubreuilh MW. Abces Intra-medullaire consecutif a une tumeur dermoide congenitale. Bull Soc Anat Physiol Norm Pathol 1887;8:13-26.

6. French BN. The embryology of spinal dysraphism. Clin Neurosurg 1983;30:295-340.

7. Hillbertz $\mathrm{NH}$. Inheritance of dermoid sinus in the Rhodesian ridgeback. J Small Anim Pract 2005;46:71-74.

8. Mclntosh R, Merritt KK, Richards MR, Samuels MH, Bellows MT. The incidence of congenital malformations: a study of 5,964 pregnancies. Pediatrics 1954;14:505-522.

9. Moise TS. Staphylococcus meningitis secondary to a congenital sacral sinus. Surg Gynecol Obstet 1926;42:394-397.

10. Nguyen HT. Intraspinal dermoid cysts in a C57BL6 mouse. Lab Anim Sci 1988;38:739-740.

11. Polo-Parada L, Bose CM, Plattner F, Landmesser LT. Distinct roles of different neural cell adhesion molecule (NCAM) isoforms in synaptic maturation revealed by analysis of NCAM $180 \mathrm{kDa}$ isoform-deficient mice. J Neurosci 2004;24:1852-1864.

12. Rilliet B, Schowing J, Berney J. Pathogenesis of diastematomyelia: can a surgical model in the chick embryo give some clues about the human malformation? Childs Nerv Syst 1992;8:310-316.

13. Ripley W, Thompson DC. Pilonidal sinus as a route of infection in a case of staphylococcus meningitis. Amer J Dis Child 1928;36:785-788.

14. Sakisaka T, Takai Y. Cell adhesion molecules in the CNS. J Cell Sci 2005;118:5407-5410.

15. Salmon Hillbertz NH, Isaksson M, Karlsson EK, Hellmén E, Pielberg GR, Savolainen P, Wade $\mathrm{CM}$, von Euler H, Gustafson U, Hedhammar A, Nilsson M, Lindblad-Toh K, Andersson L, Andersson G. Duplication of FGF3, FGF4, FGF19 and ORAOV1 causes hair ridge and predisposition to dermoid sinus in Ridgeback dogs. Nat Genet 2007;39:1318-1320.

16. Selcuki M, Vatansever S, Umur AS, Temiz C, Sayin M. Apoptosis seems to be the major process while surface and neural ectodermal layers detach during neurulation. Childs Nerv Syst 2008;24:577-580.

17. van Aalst J, Beuls EA, Cornips EM, Vanormelingen L, Vandersteen M, Weber JW, et al: Anatomy and surgery of the infected dermal sinus of the lower spine. Childs Nerv Syst 2006;22:1307-1315.

18. van Straaten HW, Jaskoll T, Rousseau AM, Terwindt-Rouwenhorst EA, Greenberg G, Shankar K, Melnick M. Raphe of the posterior neural tube in the chick embryo: its closure and reopening as studied in living embryos with a high definition light microscope. Dev Dyn 1993;198:65-76.

19. Walker E, Bucy PC. Congenital dermal sinuses: a source of spinal meningeal infection and subdural abscesses. Brain 1934;57:401-421. 


\section{Chapter 6}

\section{The intermediate type split cord malformation}

Jasper van Aalst, Emile A.M. Beuls, Johan S.H. Vles, Erwin M.J. Cornips, Henny W.M. van Straaten

Childs Nervous System 2005;21 1020-1024 


\section{Abstract}

A patient is described in which a complete osteofibrotic dorsally implanted septum was found in combination with a split cord malformation in a single dural tube. This case cannot be explained using the widely used theory as proposed by Pang et al. ${ }^{12}$ but must be regarded as a combination of a type I and II split cord malformation.

The authors state that all types of split cord malformations can be reduced to a single derailment during development, with various degrees of severity. The configuration of the malformation is determined by the way the median parts of the mesoderm come to development. Type I and II split cord malformations are not distinct entities. 


\section{Introduction}

In this report, the authors describe a patient in which a type of split cord malformation (SCM) is found that cannot be explained, using the well-known theory of Pang et al. ${ }^{12}$.

SCM is a rarely diagnosed spinal abnormality. Confusion still exists about the pathophysiology and classification of this group of spinal cord malformations.

Many terms have been used to describe the phenomenon of a part of the spinal cord being divided longitudinally into two segments. It is not yet clear whether the cord has been split or has become duplicated during development.

Several authors have tried to distinguish between diplomyelia (doubled spinal cord) and diastematomyelia (split spinal cord). The former is defined as two cords lying in one dural space, without an intervening septum. The latter is defined as two cords being housed within individual thecal sacs, separated by an osteofibrotic septum and with each cord having paramedian roots. In this report the authors will use the term 'split cord malformation', to avoid a semantic discussion.

Pang et al. ${ }^{12}$ introduced in 1992 their unified theory concerning the split cord malformation, which is generally accepted, although sometimes discussed. Bremer $^{3}$ founded this theory by stating that an accessory neurenteric canal could cause a SCM.

The author excluded persistence of the original neurenteric canal as the causal factor since most defects arise cranial to the ultimate position of this canal, being at coxygeal level.

In their unified theory, Pang et al. stated that all types of SCM originate from one basic ontogenetic error, which causes regional splitting of the neural tube and the notochord. According to this theory, an accessory neurenteric canal forms during gastrulation. In normal embryological development, the neurenteric canal, connecting the yolk sac and amnion, forms and subsequently closes. However, if a second pathological canal forms, it can cause splitting of the notochord and the overlying neural plate, thereby giving rise to a SCM.

Two types of SCM were described ${ }^{12}$. In type I SCM the two hemicords have separate arachnoidal and dural spaces. Frequently an osseous or osseocartilaginous midline septum, originating ventrally, separating the two dural sleeves is noted.

In type II SCM the two hemicords are located within one thecal sac. Type II SCM is not associated with a bony septum, although in most cases intradural bands can be found.

Which type of SCM is formed depends on the way the mesenchyme surrounds or infiltrates the fistula. 
In this report a patient is described in which a complete and dorsally implanted osteofibrotic septum was found in combination with a split cord malformation in a single dural tube. This can be seen as a combination of SCM types I and II. The authors propose that all types of SCM are part of a spectrum of malformations and not distinct entities. This description of a spectrum of malformations supports the idea of a unified theory, as proposed by Pang et al.

\section{Case report}

A 15-year old adolescent presented with walking difficulties and pain in the lower extremities. Physical examination revealed no other neurological signs or symptoms. Three years earlier she had undergone surgery because of severe scoliosis. At that time an extensive bony fusion was performed.

At first presentation in our department in 1993, computed tomography (CT) myelography showed a split cord malformation in a single dural tube, i.e. type II SCM. A dorsally situated bony spur was seen at level L3-L4, apparently indenting the dura mater, reaching halfway to the vertebral canal. Extradurally the osseous spur extended caudally into a round structure embedded in the vertebral arch (Figure 6.1).

Magnetic Resonance (MR) imaging confirmed the diagnosis of SCM in a single dural tube (Figure 6.2).

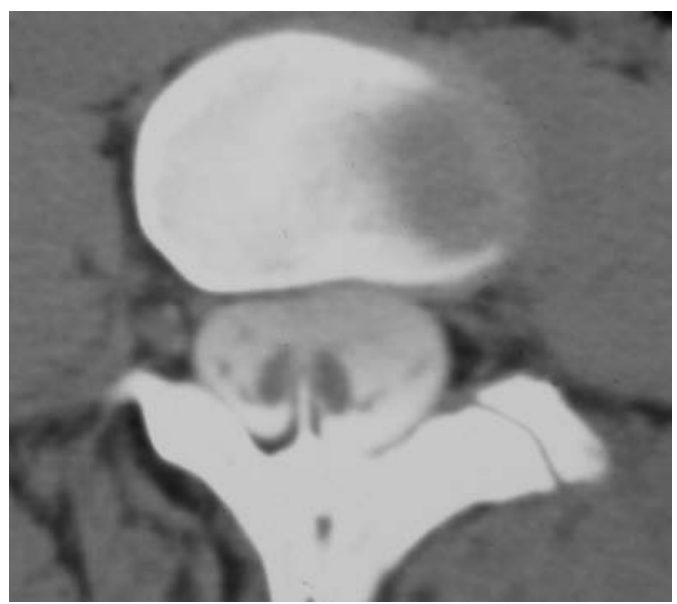

Figure 6.1 CT image showing a split cord malformation. A thick vertebral arch is seen with a dorsally originating bony spur that was interpreted as only indenting the dura. 


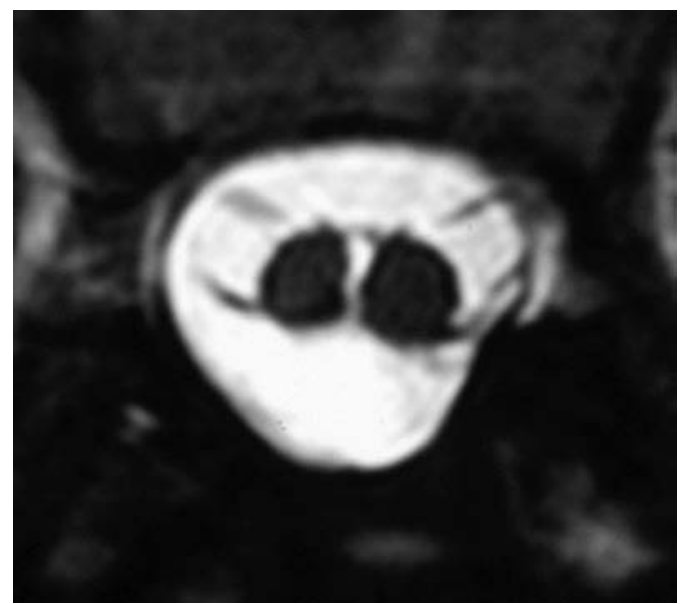

Figure 6.2 MR image showing a split cord malformation in a single dural tube.

Because of symptomatic tethering the patient was subsequently operated on. A median lumbar laminectomy was performed, without lateral extension because of the very tough bony fusion material that was found as a consequence of the scoliosis operation. Neither spur nor septum was found after intradural inspection. The filum terminale was transsected and arachnoidal adhesions between the hemicords were released. Postoperatively the patient's condition did not improve.

Two years later, MR imaging (Figure 6.3) showed a complete dorsoventral spur, tenting the cord ventrally. CT myelography revealed that the extradural part of the bony spur had been resected, whereas an intradural bony septum was still in situ, situated in the cleft between the two hemicords (Figure 6.4).

The patient was re-operated on, and a complete dorsoventral osteofibrotic septum was found with a slightly oblique course. A small part of the extradural spur was still present and was found attached to the dura mater. After opening the dura, this spur was seen traversing the dura, continuing as a bony septum into the split between the hemicords in ventrolateral direction.

The dorsal part of the septum, traversing the dura, was hard and bony up to halfway the vertebral canal whereas the ventral part was rather fibrotic up to its attachment in the ventral dura. Typical accompanying vessels ${ }^{12}$ were seen. The septum was meticulously removed in toto. Although subsequent MR imaging confirmed complete resection of the spur, the cord remained ventrally tented, even after 12 months. The patient's condition only moderately improved. 


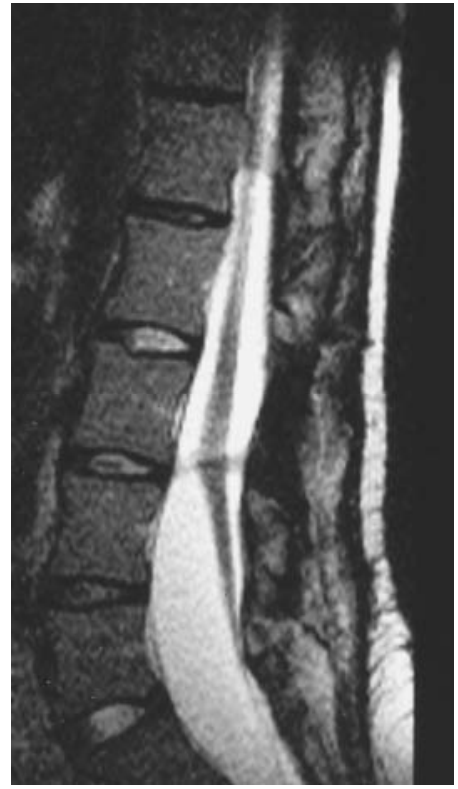

Figure 6.3 MR image made during follow-up, after the first procedure took place. The lower spinal cord is still tethered by a complete dorsoventral septum.

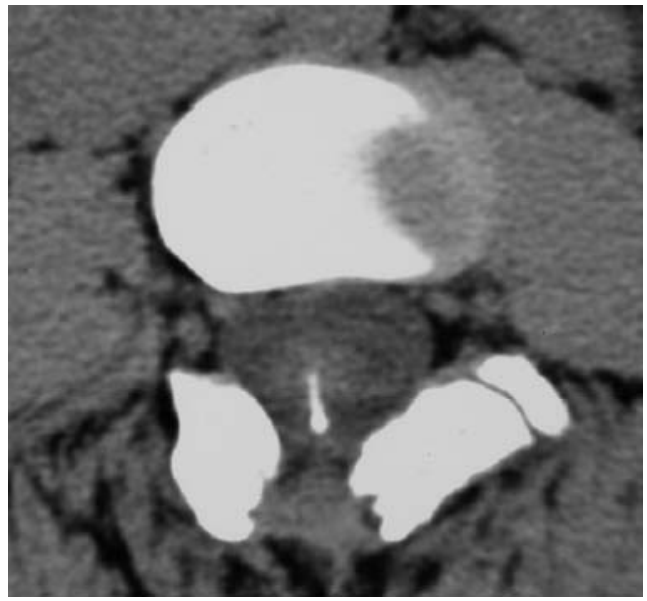

Figure 6.4 Postoperative CT image demonstrating that a limited laminectomy has been done, whereas the intradural part of the spur is still in situ. 


\section{Discussion}

In this report, a complete dorsoventral osteofibrotic septum, including a dorsally situated bony spur is described in a SCM in a single dural tube.

The intradural part of the bony septum was not found initially because the surgeon did not expect this to be present in a type II SCM. The authors assumed that the spur was only indenting the dura and that it was resected when performing the laminectomy.

According to the unified theory by Pang et al. ${ }^{12}$, a type II SCM does not coexist with a bony septum. In addition, every bony spur should have its base at the dorsal aspect of the vertebral body and not on the vertebral arch.

However, some authors have mentioned a dorsally implanted spur ${ }^{1,2,4,11}$. Chandra et al. ${ }^{4}$ reported a case of type I SCM with a dorsal bony spur indenting the dura. Ersahin et al. ${ }^{6}$ and Akay et al. ${ }^{1}$ reported on similar cases with a dorsal bony spur indenting the dura, but they found this in combination with a type II SCM. Basak et al. ${ }^{2}$ described a patient in whom a dorsally implanted spur was noted in combination with a SCM in a single dural tube, although no details were given about the spur being intradurally or only indenting the dura.

These findings indicate that the distinction between both types of SCM is not as clear-cut as stated originally. Moreover, the cause of SCM is not understood. Therefore, the developmental processes occurring in early human life might shed light on a common pathogenesis of both SCM types.

In the third week of human embryonic development, the primitive streak is rostrally bordered by the primitive knot, from which the notochord originates. With lengthening of the notochord the knot progressively shifts in caudal direction and ends up as a massive blastema called the tail bud, from which the lower part of the sacrum and the tail region arise. A temporary hole in the primitive knot, the neurenteric canal, connects the lumen of amnion and yolk sac and integrally shifts in caudal direction as well. The ectoderm becomes induced into neural plate by the underlying notochord; this plate extends in a caudal direction as well, but surpasses the primitive knot as two separate laterocaudal wings.

During the fourth week of development, the flat neural plate transforms into a tube, and becomes flanked by two rows of somites. The medial compartment of the somite, the sclerotome, dissociates into loose mesenchymal cells, which envelop both the notochord and the neural tube and form the vertebral body and arch. A mutual interaction is essential for development of all three structures $^{17}$. More importantly, the dura mater and the leptomeninges, collectively called the meninx primitiva, are postulated to arise from the inner lining of this mesenchymal envelope ${ }^{9,15}$.

Various mechanisms causing SCM have been postulated. By a persistent neurenteric canal, the caudal wings of the neural plate will remain separated 
and develop into two neural tubes. This malformation has to occur at a caudal level only since the neurenteric canal ends up in the tail bud.

An additional canal is postulated as well. In amphibian neurula stage embryos median incision of the neural plate and notochord resulted in some embryos with $\mathrm{SCM}^{5}$. In an experiment by Rilliet et al. ${ }^{13}$ a sagittal incision at the level of the rhomboidal sinus in 2-day-old chick embryos was performed. When the gap was kept open with an agar screen or without any intervening substrate, no SCM developed. When a piece of membranous egg shell or of notochord was inserted to create a permanent separation, more than half of the embryos displayed SCM, each hemicord having its own central canal. It thus seems that a schisis of the neural plate has to occur, and has to be open long enough to allow full closure of the neural plate halves. In the human embryo, (para) medial schisis of the already existing neural plate might occur, e.g. due to unequal pressure between amnion and yolk sac. Such a gap is not necessarily restricted to the caudal region.

A third possiblity is a local "Siamese" condition, due to a temporary splitting of the primitive knot. In this case, two notochords and two neural tubes arise and depending on the grade of separation, somites will develop in between as well. In an experimental setting, inplantation of a somite into a just closed part of the neural tube in a chick embryo resulted in $\mathrm{SCM}^{10}$.

Therefore, from these experiments no conclusion can be drawn about the origin of the double neural tube: whether a single neural plate is medially separated or whether two parallel neural tubes come to development.

The development of the midline septum, dura mater and a bony spur are enigmatic as well. In a type I SCM, meninx primitiva cells are said to arise ventrally and to subsequently migrate dorsally along the mesenchymal septum. This subsequently leads to the formation of dura mater in between the two hemicords. Also other derivatives can form in the midline septum, such as bony or cartilaginous structures. The formation of a bony septum is induced by the osteogenic potential of the developing dura. The outer lining of the meninx primitiva cells induces formation of the bony part of the septum. Therefore this septum will always be extradural. When the meninx primitiva cells arise dorsally and start migrating in a ventrolaterally direction ${ }^{15}$, the bony spur, if present, will have its origin at the dorsal site of the vertebral body.

In a type II SCM, the mesenchyme surrounding the fistula does not develop meninx primitiva cells. Therefore no dura mater or bony spur is formed in the midline ${ }^{12}$.

As demonstrated in this case and many others ${ }^{1,2,4,6-8,11,14,16}$, many variations can be found, as well as combinations of the two types of SCM.

The authors agree with other authors that all variants of SCM might be caused by the same mechanism. An accessory neurenteric canal, as proposed by Bremer $^{3}$ and Pang et al. ${ }^{12}$, seems the most likely candidate for the initiation of 
SCM. We propose that in all above cases, the mesenchymal cells from the sclerotome will envelop both tubes. The larger the amount of cells in between, the better the median parts of both tubes come to development, the better the meninges form and the better a septum will be formed, ultimately including development of a bony spur. A pathological development is seen in the midline where a chaotic pattern of differentiation will take place. The development of the median parts of the neural tube is dependent of the amount of precursor cells available and as is described in this case, a bony septum can develop without the presence of an inducing midline dura.

This implies that SCM types I and II are part of a spectrum of malformations, all caused by the same ontogenetic error. The SCM described in this report demonstrates the possibility of an intermediate-type SCM and supports the theoretical considerations given in this report.

\section{Conclusion}

In case of a split cord malformation in a single dural tube, a dorsally implanted bony spur can be found continuing intradurally into a complete intradural osteofibrotic septum. All types of split cord malformation might be caused by the same ontogenetic error, leading to a pathological development of the median parts of the mesoderm. All types of SCM can be interpreted as degrees of severity in which the malformation took place. 


\section{References}

1. Akay KM, Izci Y, Baysefer A. Dorsal bony septum: a split cord malformation variant. Pediatr Neurosurg 2002;36:225-228.

2. Basak M, Ozel A, Erturk M. An unusual case of diastematomyelia. Presence of one dura sheath associated with a bony spur. Acta Radiologica 2002;43:626.

3. Bremer J. Dorsal intestinal fistula; accessory neurenteric canal; diastematomyelia. Arch Pathol 1952;54:53-54.

4. Chandra PS, Kamal R, Mahapatra AK. An unusual case of dorsally situated bony spur in a lumbar split cord malformation. Pediatr Neurosurg 1999;31:49-52.

5. Emura T, Asashima M, Furue M, Hashizume K. Experimental split cord malformations. Pediatr Neurosurg 2002;36:229-235.

6. Ersahin Y. An unusual split cord malformation. Pediatr Neurosurg 2000;32:109.

7. Erşahin Y, Demirtaş E, Mutluer S, Tosun AR, Saydam S. Split cord malformations: report of three unusual cases. Pediatr Neurosurg 1996;24:155-159.

8. Erşahin Y, Mutluer S, Kocaman S, Demirtaş E. Split spinal cord malformations in children. J Neurosurg 1998;88:57-65.

9. Halata Z, Grim M, Christ B. Origin of spinal cord meninges, sheaths of peripheral nerves, and cutaneous receptors including Merkel cells. Anat Embryol 1990;182:529-537.

10. Klessinger S, Christ B. Diatematomyelia and Spina Bifida Can Be Caused by the Intraspinal Grafting of Somites in Early Avian Embryos. Neurosurgery 1996;39:1215-1223.

11. Leys D, Samain F, Lesoin F, Franz K, Viaud C, Petit H. "Partial diastematomyelia" in an adult with a posterior bony spur. Rev Neurol (Paris) 1987;143:63-67.

12. Pang D, Dias MS, Ahab-Barmada M. Split cord malformation: Part I: A unified theory of embryogenesis for double spinal cord malformations. Neurosurgery 1992;31:451-480.

13. Rilliet B, Schowing J, Berney J. Pathogenesis of diastematomyelia: can a surgical model in the chick embryo give some clues about the human malformation? Childs Nerv Syst 1992;8:310-316.

14. Schijman E. Split spinal cord malformationsReport of 22 cases and review of the literature. Childs Nerv Syst 2003;19:96-103.

15. Sensenig EC. The early development of the meninges of the spinal cord in human embryos. Contr Embryos 1949;33:21-41.

16. Vaishya S, Kumarjain P. Split cord malformation: three unusual cases of composite split cord malformation. Childs Nerv Syst 2001;17:528-530.

17. van Straaten HWM, Hekking JWM. Development of floor plate, neurons and axonal outgrowth pattern in the early spinal cord of the notochord-deficient chick embryo. Anat Embryol 1991;184:55-63. 


\section{Chapter 7}

\section{The Currarino triad: neurosurgical considerations}

Pieter J. Emans, Jasper van Aalst, Ernest L.W. van Heurn, Carlo Marcelis, Gauke Kootstra, Regina G.H. Beets-Tan, Johan S.H. Vles, Emile A.M. Beuls Neurosurgery 2006;58:924-929 


\section{Abstract}

\section{Objectives}

The Currarino triad, a relatively uncommon hereditary disorder, is often associated with a tethered cord and anterior myelomeningocele. Little is known of the implications of these neuroanatomical malformations or of the neurosurgical attitude.

The objective of this study is to identify the spinal cord and meningeal malformations associated with the Currarino triad and to discuss the risks and benefits of surgical intervention.

\section{Methods}

We analyzed the spinal cord malformations and the neurosurgical involvement with the Currarino triad by retrospective chart review.

\section{Results}

The Currarino triad neuroanatomic malformations were identified in five patients. The Currarino triad was associated with a tethered cord in three patients, a myelomeningocele in five patients, a syrinx in two patients, a fistula between the colon and spinal canal in two patients, and an ArnoldChiari type 1 malformation in one patient.

\section{Conclusion}

Full spine imaging is required for all patients diagnosed with the Currarino triad. Magnetic resonance imaging of the head should be performed in every patient with neuroanatomical anomalies. Surgery of an anterior myelomeningocele is not necessarily indicated, only in the rare case in which the space-occupying aspect is expected to cause constipation or problems during pregnancy or delivery. Constipation directly after birth is seen in virtually all patients with the triad. Therefore, constipation cannot be used to diagnose a tethered cord syndrome nor indicate tethered cord release. Fistulas between the spinal canal and colon have to operated on directly. 


\section{Introduction}

The Currarino triad is an autosomal dominant disorder linked to the $7 q 36$ region ${ }^{5,10,12}$. It consists of a bony sacral defect, an anorectal malformation, and a presacral mass ${ }^{1,6}$. In most patients, this mass is a teratoma, an anterior myelomeningocele (AMMC), or a combination of both ${ }^{4,6,8,17}$. The incidence of the Currarino triad is unknown. It is often not recognized, and fewer than 250 patients with the triad have been reported until now ${ }^{6,13}$. The neurosurgical consequences are almost unknown ${ }^{3,14}$.

The triad is often associated with other disorders such as a tethered cord (TC), duplication of the urogenital tract, and different types of fistulas, such as a fistula between the colon and spinal canal, which may cause meningitis ${ }^{4,6,8,17}$. Most patients with the Currarino triad are constipated. The cause remains controversial $^{4,6,8,16}$. Some think TC and AMMC may cause constipation and, therefore, should be treated ${ }^{11,16}$. We describe five patients with the Currarino triad with neuroanatomical malformations, and we discuss the indications and cautions of neurosurgical treatment.

\section{Patients and methods}

In the time period between 1994 and 2003, five patients with the Currarino triad and spinal or cerebral malformations on magnetic resonance imaging (MRI) evaluation presented at the University Hospital Maastricht. The presenting symptoms were constipation, meningitis, and neurogenic claudication. The neurosurgical considerations and sequela are described below.

\section{Patients}

\section{Patient 1}

A 1-month-old girl was admitted to the hospital with meningitis. Apart from severe constipation, her medical history was unremarkable. Her meningitis was caused by $E$. coli and was treated with antibiotics. At a routine medical examination, there was a narrow anal canal, which could not be entered with the little finger. There were no neurological abnormalities. Hirschsprung's disease was excluded by rectal biopsy. Ultrasonography and MRI scans showed a presacral mass, partially consisting of an AMMC, sacral agenesis, and a TC with the conus of the spinal cord at sacral level. A fistula between the spinal canal and colon could not be visualized. The diagnosis of Currarino triad was made. The patient received a colostomy of the transverse colon, and the 
presacral mass was resected through a posterior sagittal operation. The most caudal part of the mass was attached to the spinal canal.

Histology of the mass was benign teratoma, which was attached to an AMMC. After closure of the colostomy, the patient still experienced chronic constipation and recurrent urinary tract infections. A urodynamic investigation (UDI) established that there was urinary retention. Ten years after operation, the symptoms of the patient have not changed. She is still incontinent for both urine and feces. The cause of the incontinence remains unknown and may be caused by the triad itself or by the resection of the presacral mass. Despite neonatal TC, the patient has not developed sensory deficit or gait abnormalities as symptoms of TC syndrome (TCS).

\section{Patient 2}

A 1-week-old girl (sister of patient 1) presented with anal blood loss and constipation. Upon physical and neurological examination, the anal canal was narrow, and there was a sacral dimple and a cortical thumb. At study of a plain $\mathrm{x}$-ray, there was hypoplasia of the sacral bone. MRI scans of the pelvis showed a thickened puborectal sling, which caused the anal stenosis, a solid presacral mass associated with a small AMMC of less than $1 \mathrm{~cm}$ in the defect of the sacrum, a low position of the conus of the spinal cord at level L2-L3, and a thickened filum terminale. The solid part of the tumor was resected by a team of pediatric and neurosurgeons, without opening the dura mater. The TC was not symptomatic, and no TC release was performed. Histopathology of the mass showed a mature benign teratoma. Postoperatively, and during follow-up $(5 \mathrm{yr})$, the patient was severely constipated. There was also urinary retention with an increased activity of the pelvic muscles at the UDI. Because no preoperative UDI had been performed, it is unknown whether there was intraoperative nerve damage.

\section{Patient 3}

A 1-week-old girl presented with vomiting and constipation. There was a distended abdomen and a narrow anal canal. Neurologically, the neonate was normal. Plain and contrast radiography showed a sciatiform sacral bone, a dilated sigmoid colon, and a rectovaginal fistula. Hirschsprung's disease was excluded with a biopsy. MRI scans of the pelvis showed a ventrally displaced rectum and a presacral mass attached to an AMMC. A spinal MRI scan showed a small syrinx at Th9-Th12 with the conus of the spinal cord at level L1. The presacral mass was resected through a posterior sagittal approach with a temporary colostomy. The AMMC was left in place. Histopathological examination confirmed the diagnosis of a mature teratoma. At the age of six years, the patient is incontinent for feces and has recurrent urinary tract infections caused by loss of detrusor activity. 


\section{Patient 4}

A 25-year-old woman presented with plans to conceive. Her medical history included a presacral mass, a perianal fistula, and a ventrally displaced anus, which were diagnosed during anorectal examination at the age of two years. An attempt was made to resect the tumor during childhood, but this was abandoned because the tumor proved to be an AMMC. Twenty-two years later, she returned to the hospital with complaints of a neurogenic claudication, which suggested a TCS. UDI showed instable contractions of the detrusor muscle. Neurological examination revealed an absent bulbocavernosus reflex, which indicated a lesion of the pudendus nerve. MRI scans showed an Arnold-Chiari Type 1 malformation, a syrinx at the C6-C7 level, and a TC with the conus at the level of S2, which was attached to a large AMMC (Figures 7.1-7.3).

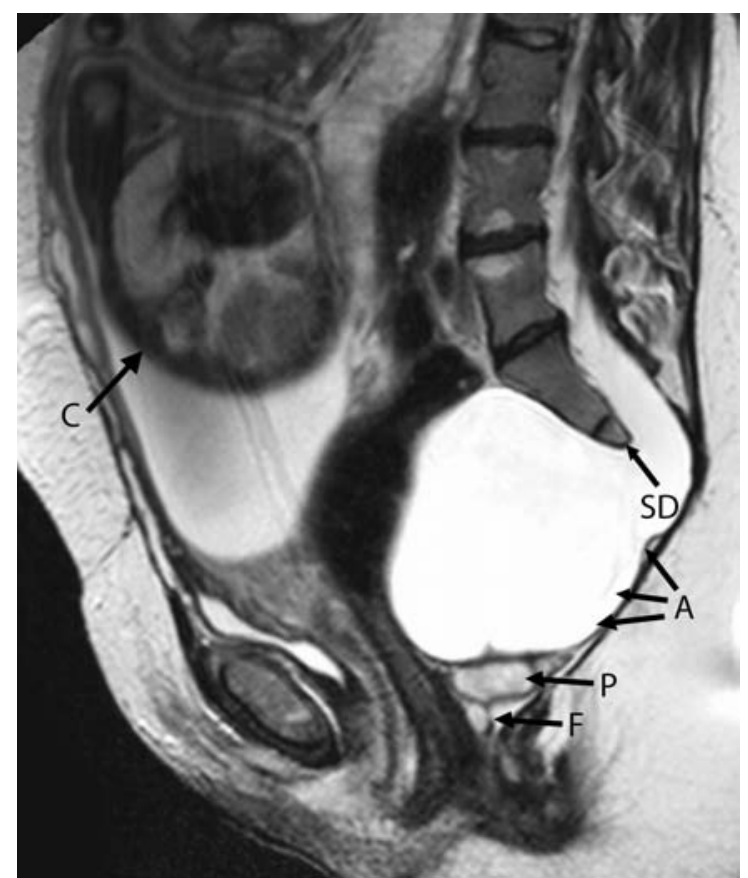

Figure 7.1 MR image of patient 4 at 34 weeks of pregnancy. This patient presented with 1. A ventrally displaced anus and constipation (not shown), 2. A large anterior myelomeningocele $(A)$, and 3 . A sacral defect $(\mathrm{SD})$. A remnant fistula $(F)$ with a fluid pocket $(P)$ and the child in utero $(C)$ are also shown 
Because the TC was symptomatic, an attempt was made for surgical untethering. Preoperatively, we considered a release of the filum terminale. However, a tapered conus was found to be firmly attached to the dura. Also several, possibly essential, blood vessels were seen. Due to these blood vessels and the explicit wish of the patient not to take the slightest risk, the cord was not untethered and neither was the AMMC resected. No change in signs or symptoms were observed during a follow-up period of 6 years, and the patient had two uncomplicated pregnancies and vaginal deliveries. Family screening revealed that her mother had an incomplete Currarino triad and a cousin had a complete Currarino triad (patient 5).
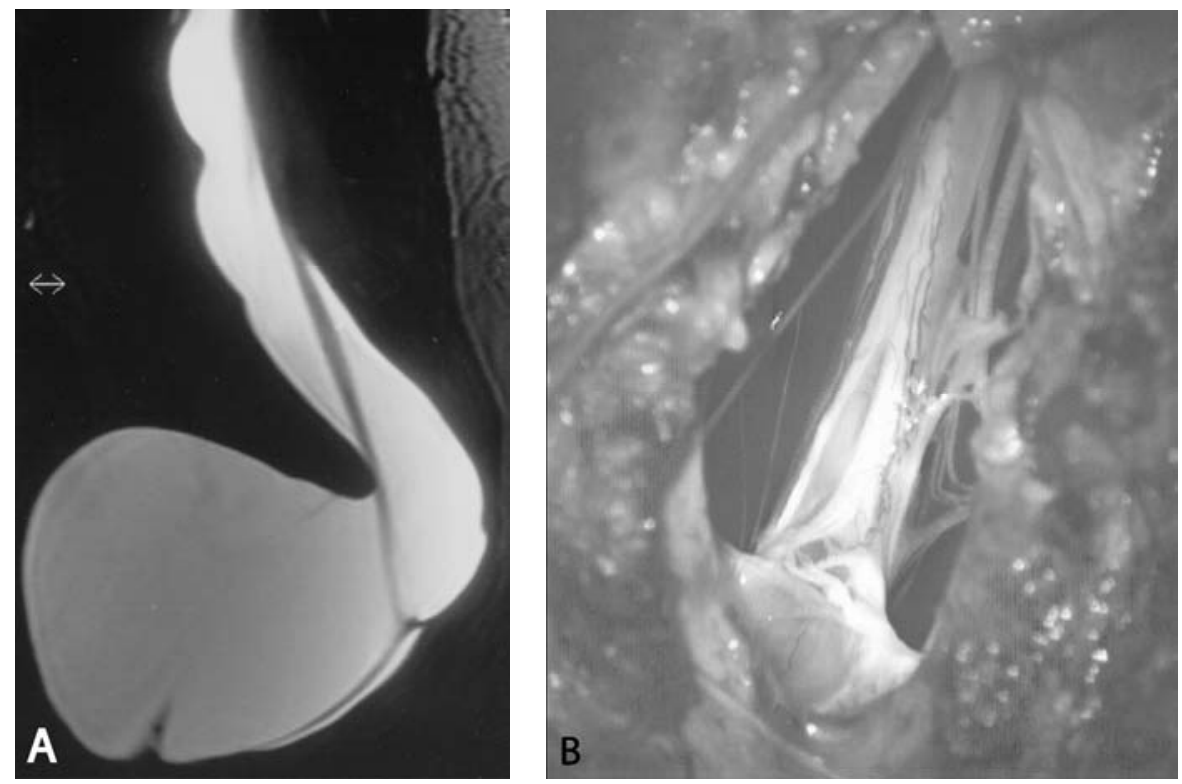

Figure 7.2 A. Preoperative MR image of AMMC. B. Intraoperative view of AMMC. Both panels show the complex anatomy at the site of adherence of the lower spinal cord to the dura mater.

See page 159 for colour figure.

\section{Patient 5}

A 27-year-old woman presented with plans to conceive. Her family was known to have the Currarino triad. Apart from chronic constipation since childhood, her medical history was unremarkable. A gynaecological examination revealed an intra-abdominal mass; neurological examination showed no abnormalities. A roentgenogram showed a hypoplastic sacrum and an absent coccygeal bone. MRI scans showed a bicornic uterus and a mass with the characteristics of a dermoid tumor, which was attached to a large AMMC. The position of the 
conus was at Th12-L1. Because of her plans to conceive, both the AMMC and the attached tumor were excised through a combined anterior and posterior approach by a team of neuro- and gastrointestinal surgeons. The histopathological diagnosis was of a dermoid tumor attached to an AMMC. After a follow-up period of 5 years, the patient is still constipated without other symptoms.

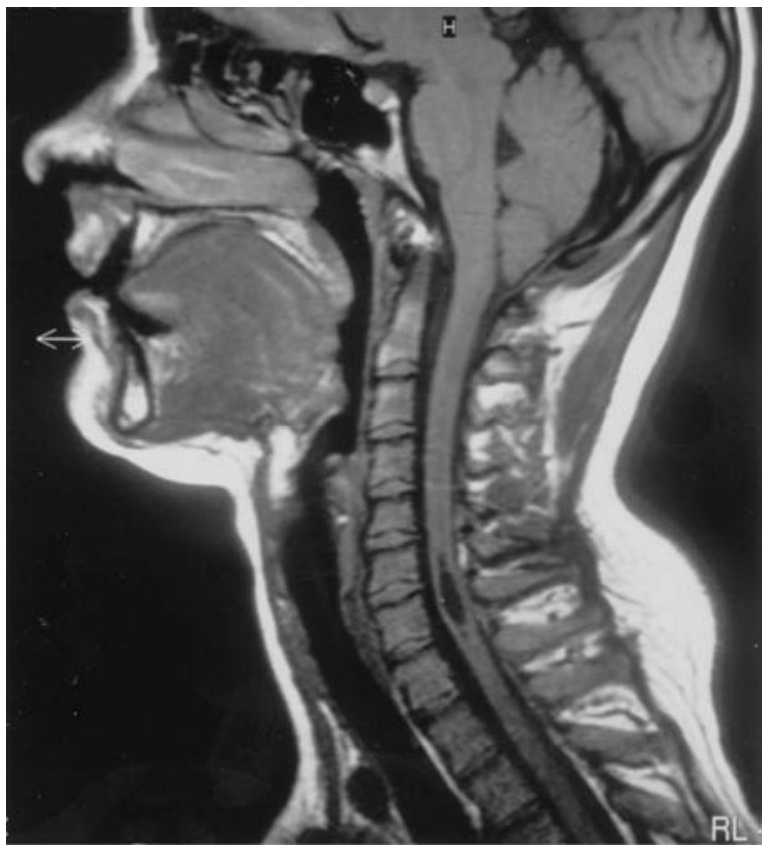

Figure 7.3 MR image of patient 4 showing a syrinx at the C6-C7 level and a type 1 ArnoldChiari malformation.

\section{Discussion}

\section{Etiology}

The Currarino triad was originally described as a combination of an anorectal malformation, a presacral mass, and sacral bone abnormalities ${ }^{1}$. It is caused by an autosomal dominant genetic defect, which is linked to the $7 q 36$ region ${ }^{10}$. This linkage has recently been refined to a homeobox gene HLXB9 as the major gene for inherited sacral agenesis ${ }^{12}$. Genetic screening for mutations in different exons of this gene is still difficult ${ }^{5}$. Because the affected sacral 
elements are always below the level of $\mathrm{S} 1$, the authors agree with Gegg et al. ${ }^{3}$, who state that errors in migration of the pluripotent caudal eminence after primary neurulation can cause the anomalies seen in the Currarino triad and that the teratogenic mass could be a remnant of the caudal eminence itself.

\section{Incidence}

The triad is often associated with neuroanatomical defects. In approximately 60 $\%$ of the patients, the Currarino triad is associated with an $\mathrm{AMMC}^{6,13}$. It may be without symptoms and may be diagnosed late in life, usually during gynaecological examination ${ }^{6,14}$. A TC is reported in approximately $18 \%$ of the patients with the Currarino triad. This incidence is lower than the incidence of a TC in other diseases associated with anorectal and sacral malformations ${ }^{9,11}$. Therefore, the real incidence of a TC in patients with the Currarino triad may be found to be higher if an active search for spinal anomalies is performed.

Meningitis is a relatively rare complication of the Currarino triad and has only been reported in a few patients ${ }^{6,17}$. It may be caused by a fistula from the bowel to an AMMC or to the spinal canal. An overview of the neuroanatomical anomalies associated with the Currarino triad is shown in Table 7.1.

\section{Diagnosis}

The diagnosis of a Currarino triad should be considered in children with severe constipation after birth with or without an anorectal malformation. A positive family history is supportive of the diagnosis; however, if the family history is negative for the Currarino triad, the diagnosis cannot be excluded ${ }^{2}$. The triad is generally diagnosed with a plain x-ray or ultrasound of the sacral bone. Any variation of a sacral bone defect may confirm the diagnosis. If the diagnosis is made, imaging of the presacral region and the full spine is necessary. In addition, MRI scans of the head are required in every patient with neuroanatomical malformations. In children aged three months or younger, the vertebral bodies are not yet calcified, and ultrasonography can diagnose a possible presacral mass at the level of the conus medullaris. In older patients, $\mathrm{MRI}$ is the investigative method of choice. With recent techniques, it is possible to visualize tumors in great detail and to diagnose spinal abnormalities. Even perianal fistulas can be visualized by this technique ${ }^{15}$ (Figure 7.1).

For patients with a TC or AMMC, MRI investigation must be extended to the head and full spine to diagnose a possible syrinx or Arnold-Chiari malformation (Patient 4). To exclude a symptomatic TC, frequent UDI, especially pre- and postoperatively, is mandatory in every patient with the Currarino triad. 
Table 7.1 Neuroanatomical anomalies associated with the Currarino triad. ${ }^{\text {a }}$

\begin{tabular}{|c|c|c|}
\hline Neuroanatomical malformations & Assumed pathogenesis & Treatment \\
\hline Arnold-chiari malformation Type 1 & $\begin{array}{l}\text { Increased craniospinal CSF pressure } \\
\text { gradient }\end{array}$ & $\begin{array}{l}\text { Conservative if clinically } \\
\text { asymptomatic }\end{array}$ \\
\hline AMMC & $\begin{array}{l}\text { Misdirection and disorganization of } \\
\text { the caudal eminence (3) }\end{array}$ & $\begin{array}{l}\text { Individualized approach surgery is } \\
\text { considered if space occupying aspect } \\
\text { may result in constipation or } \\
\text { problems during pregnancy or } \\
\text { delivery }\end{array}$ \\
\hline Tethered cord & $\begin{array}{l}\text { Thickened filum terminale, } \\
\text { intramedullary lipomatous mass }\end{array}$ & $\begin{array}{l}\text { Constipation at birth is no indication } \\
\text { for surgical release; surgery is } \\
\text { considered as child grows and } \\
\text { tethered cord becomes symptomatic }\end{array}$ \\
\hline $\begin{array}{l}\text { Fistulas between colon and spinal } \\
\text { canal }\end{array}$ & $\begin{array}{l}\text { Misdirection and disorganization of } \\
\text { the caudal eminence ( } 3 \text { ) }\end{array}$ & $\begin{array}{l}\text { Immediate surgical treatment to } \\
\text { prevent life-treating meningitis }\end{array}$ \\
\hline
\end{tabular}

${ }^{\text {a }}$ AMMC, anterior myelomeningocele; CSF, cerebrospinal fluid.

\section{Treatment}

\section{Anterior myelomeningocele}

None of the older patient had symptoms that could be attributed to an AMMC (Patients 4 and 5), indicating that, during childhood, an AMMC should be treated conservatively. Resection of a presacral mass during childhood may cause iatrogenic damage, causing incontinence and urinary retention (Patients 1-3). Therefore, the authors think routine resection of an AMMC is not necessary because they are usually without symptoms. Theoretically, abortion, premature birth, or pressure on the central nervous system may complicate pregnancy and delivery. We show that uneventful pregnancy and vaginal delivery is possible even when the AMMC is large (Patient 4).

The cause of constipation in the Currarino triad is unknown. TC and a presacral mass effect (including the AMMC) have been suggested as possible causes ${ }^{16}$. Constipation is diagnosed in more than $95 \%$ of the patients with the Currarino triad, irrespective of a present TC or AMMC ${ }^{1,2,6,8}$. Therefore, it is difficult to decide whether an AMMC or TC is symptomatic with constipation as the only presenting symptom. Because resection of a presacral mass has not relieved patient from constipation, the authors believe that constipation is probably not caused by an AMMC in the Currarino triad, and the cause of constipation remains unknown. Because malignant teratomas in patients with the Currarino triad have been reported in the literature, solid tumors have to be resected unless the diagnosis of teratoma can be excluded ${ }^{6,17}$. If resection of a solid tumor is combined with a resection of an AMMC, a combined anterior and posterior approach may facilitate the procedure. This is an approach to remove 
the solid tumor ventrally and close to the AMMC posteriorly, dissecting the spinal arachnoid membrane from the massive intra-abdominal AMMC.

\section{Tethered cord}

TC, defined as a conus medullaris below the level of L2, is only released if there is a TCS. The diagnosis of a TCS is made clinically. Presenting symptoms are bladder dysfunction, sensory deficit in the lower extremities, abnormal gait, or pain the back, legs, or feet.

There may a difference in opinion as to which symptoms of the Currarino triad can be attributed to a $\mathrm{TC}^{4,6,8,16}$. Constipation is also present in virtually all patients without a TC (Patients 3 and 5$)^{2,6,8}$. Therefore, constipation cannot be used to diagnose a TCS, or to indicate the release of a TC. Lagae et al. ${ }^{7}$ agree that surgical treatment is not indicated for a low-positioned conus without progressive signs and symptoms. Constipation is present at birth and is expected to be caused by the triad itself. Therefore, the authors believe that constipation at birth is not a presenting symptom of a TCS. Because no progressive signs and symptoms were observed in patients 1 to 3 during follow-up, urinary retention or urinary incontinence and abnormalities found during UDI were not regarded to be caused by a TC. Moreover, an abnormal UDI was also found in a patient without a TC (Patient 3). No TC release was performed during childhood, and none of these patients developed symptoms during follow-up that could be attributed to a TC (Patients 1 and 2). TC release should only be performed if there is a clear development of a TCS. The cause of the postoperative urinary problems in these patients remains unknown and might have been caused by surgery on the triad itself. To detect possible neurological deterioration and tethering, or retethering, frequent neurological examination and UDI should be performed ${ }^{16}$.

In patients with the Currarino triad, incomplete ascensus of the conus medullaris to the normal level of Th12-L1 may be caused by anchorage by a shortened filum terminale or lipomatous mass. The conus medullaris may also be embedded in the complex anatomy of a neural tube/mesodermal closure defect. Because of this complex anatomy, complications after TC release in these patients could be more frequent.

\section{Fistulas between gastrointestinal tract and spinal canal}

Fistulas between the intradural space and the bowel should be closed immediately to prevent a life-threatening meningitis. In patient 1 , a meningitis was diagnosed and successfully treated with antibiotics. In this patient, the presence of a fistula could not be excluded, although it could not be visualized. After antibiotic treatment of meningitis, the child underwent resection of a presacral teratoma and received a temporary colostomy. Both may have resulted in closure of a fistula. 


\section{Conclusion}

In all patients with the Currarino triad, an active search for spinal cord anomalies is advised, including a complete medical history, full neurological examination, and imaging of the spine that is extended to the head if neuroanatomical malformations (e.g. TC or AMMC) are present. There is no indication for routine TC release because several of these symptoms may be attributed to the triad itself. TC release should be considered if symptoms are progressive because of conus involvement, causing a TCS. Because constipation is present in virtually every patient with the triad with or without a TC, constipation cannot be used to diagnose a TCS or to indicate the release of a TC.

There is no evidence that an AMMC, if present, has to be resected. Theoretically, it may give such symptoms as constipation and problems during delivery, but vaginal delivery remains possible, even if the AMMC is large. Therefore, we advise an multidisciplinary and individualized approach. 


\section{References}

1. Currarino G, Coln D, Votteler T. Triad of anorectal, sacral, and presacral anomalies. AJR Am J Roentgenol 1981;137:395-398.

2. Emans PJ, Kootstra G, Marcelis CL, Beuls EA, van Heurn LW. The Currarino triad: the variable expression. J Pediatr Surg 2005;40:1238-1242.

3. Gegg CA, Vollmer DG, Tullous MW, Kagan-Hallet KS. An unusual case of the complete Currarino triad: case report, discussion of the literature and the embryogenic implications. Neurosurgery 1999;44:658-662.

4. Heij HA, Moorman-Voestermans CG, Vos A, Kneepkens CM. Triad of anorectal stenosis, sacral anomaly and presacral mass: a remediable cause of severe constipation. $\mathrm{Br} \mathrm{J}$ Surg 1990;77:102-104.

5. Kochling J, Karbasiyan M, Reis A. Spectrum of mutations and genotype-phenotype analysis in Currarino syndrome. Eur J Hum Genet 2001;9:599-605.

6. Kochling J, Pistor G, Marzhauser Brands S, Nasir R, Lanksch WR. The Currarino syndrome-hereditary transmitted syndrome of anorectal, sacral and presacral anomalies. Case report and review of the literature. Eur J Pediatr Surg 1996;6:114-119.

7. Lagae L, Verpoorten C, Casaer P, Vereecken R, Fabry G, Plets C. Conservative versus neurosurgical treatment of tethered cord patients. Z Kinderchir 1990;45(Suppl 1):16-17.

8. Lee SC, Chun YS, Jung SE, Park KW, Kim WK. Currarino triad: anorectal malformation, sacral bony abnormality, and presacral mass--a review of 11 cases. J Pediatr Surg 1997; 32:58-61.

9. Levitt MA, Patel M, Rodriguez G, Gaylin DS, Pena A. The tethered spinal cord in patients with anorectal malformations. J Pediatr Surg 1997;32:462-468.

10. Lynch SA, Bond PM, Copp AJ, Kirwan WO, Nour S, Balling R, Mariman E, Burn J, Strachan $\mathrm{T}$. A gene for autosomal dominant sacral agenesis maps to the holoprosencephaly region at 7q36. Nat Genet 1995;11:93-95.

11. Pang D. Sacral agenesis and caudal spinal cord malformations. Neurosurgery 1993;32:755778; discussion 778-759.

12. Ross AJ, Ruiz-Perez V, Wang Y, Hagan DM, Scherer S, Lynch SA, Lindsay S, Custard E, Belloni E, Wilson DI, Wadey R, Goodman F, Orstavik KH, Monclair T, Robson S, Reardon W, Burn J, Scambler P, Strachan T. A homeobox gene, HLXB9, is the major locus for dominantly inherited sacral agenesis. Nat Genet 1998;20:358-361.

13. Samuel M, Hosie G, Holmes K. Currarino triad--diagnostic dilemma and a combined surgical approach. J Pediatr Surg 2000;35:1790-1794.

14. Villarejo F, Scavone C, Blazquez MG, Pascual-Castroviejo I, Perez-Higueras A, FernandezSanchez A, Garcia Bertrand C. Anterior sacral meningocele: review of the literature. Surg Neurol 1983;19:57-71.

15. Vliegen RF, Beets-Tan RG, van Heurn LW, van Engelshoven JM. High resolution MRI of anorectal malformation in the newborn: case reports of Currarino syndrome and anocutaneous fistula. Abdom Imaging 2002;27:344-346.

16. Walton M, Bass J, Soucy P. Tethered cord with anorectal malformation, sacral anomalies and presacral masses: an under-recognized association. Eur J Pediatr Surg 1995;5:59-62.

17. Yates VD, Wilroy RS, Whitington GL, Simmons JC. Anterior sacral defects: an autosomal dominantly inherited condition. J Pediatr 1983;102:239-242. 


\section{Intraspinal dermoid and epidermoid tumors: report of 18 cases and reappraisal of the literature}

Jasper van Aalst, Franka Hoekstra, Emile A.M. Beuls, Erwin M.J. Cornips, Jacobiene W. Weber, Deborah A Sival, David HKV Creytens, Johan S.H. Vles Pediatric Neurosurgery 2009;45:281-290 


\section{Abstract}

Intraspinal dermoid and epidermoid tumors are two histopathological subtypes of cutaneous inclusion tumors of the spine. This classification is based on obsolete embryological knowledge. In fact, according to current embryology, both tumor types consist of ectodermal derivatives. Therefore, we hypothesized that dermoid and epidermoid tumors do not differ in clinical practice. To explore this hypothesis, we studied the clinical, radiological and intraoperative findings of 18 patients, and related these findings to the histopathological characteristics of the tumor. No differences were found between dermoid and epidermoid tumors regarding clinical presentation, radiological examination and outcome, while intraoperative diagnosis by the surgeon correlated with the histopathological diagnosis in only 8 of 18 cases. Therefore, the histopathological difference between intraspinal dermoid and epidermoid tumors is not important in clinical practice and should be avoided. A new nomenclature is proposed in which both tumor types are referred to as 'spinal cutaneous inclusion tumors'. 


\section{Introduction}

Dermoid and epidermoid tumors are rare cutaneous inclusions within the central nervous system. If present, they are mainly localized in the brain ${ }^{19,27}$. Also, dermoid tumors are more common in the spine ${ }^{19,33,51}$, and epidermoid tumors in the cranium ${ }^{19,26,33,51}$. The nomenclature of these tumors has been subject of debate for many years. After several descriptive definitions were proposed, Sachs and Horrax suggested in 1949 to differentiate spinal dermoid from epidermoid tumors by the presence of dermis and 'dermal glands ${ }^{\prime 45}$. The etiology of spinal dermoid and epidermoid tumors is not exactly understood. Their origin might be acquired or congenital. Acquired or iatrogenic tumors may arise from implantation of epidermal tissue into the subdural space during needle puncture $e^{8,10,28,31,41,42,47,57,60}$ or during surgical procedures s $^{9,21,30,35,37,46,50}$. Congenital dermoid and epidermoid tumors may develop from displaced cutaneous ectoderm during neural tube formation ${ }^{11,12,25,28,43,45,52}$. The clinical presentation of a patient with a spinal dermoid or epidermoid tumor depends on the location and extend of the tumor. Motor disturbances, pain, sensory disturbances, and urological dysfunction are frequently reported symptoms. The literature on magnetic resonance (MR) characteristics of intraspinal dermoid and epidermoid tumors is inconclusive ${ }^{1,8,15,18,20,22,29,32,36,39,49,53,57,59}$. Both tumors may display a variety of signal intensities on T1- and T2-weighted MR images.

The literature on intraspinal dermoid and epidermoid tumors comprises only case reports and a limited number of small series of cases, describing 16 patients maximum ${ }^{2,3,19,23,27,40,48}$.

Although the difference in etiology is not known, and in fact the name 'dermoid' is because of its contents a misnomer ${ }^{13,24}$, it is still custom to distinguish dermoid and epidermoid tumors in clinical practice. However, this classification is based on outdated embryological knowledge. In fact, according to current embryological knowledge, both tumor types consist of ectodermal derivatives. Therefore, we hypothesized that the difference between dermoid and epidermoid tumors can only be made after histopathological examination, and is not of significance in clinical practice. The aim of our study is, to describe the clinical, radiological and intraoperative findings of 18 patients, to the best of our knowledge the largest series of intraspinal dermoid/epidermoid tumors, and relate these findings to the gold standard for diagnosis of all tumors: histopathological examination. 


\section{Patients and methods}

Within the two hospitals participating (University Medical Center Maastricht and University Medical Center Groningen), eligible patients were collected from several databases. Spinal dermoid and epidermoid tumor cases from the past 15 years were retrospectively reviewed, including in- and outpatient medical charts, radiographs, operative notes and pathology specimens.

Tumor signal intensities on preoperative T1-weighted and T2-weighted MR images were independently assessed by two of the authors (J.S.H.V., J.v.A.). All pathology specimens were microscopically reassessed by a single neuropathologist (DC), and this histopathological reassessment was defined as the 'gold standard'.

A dermoid tumor was diagnosed if squamous stratified epithelium, desquamated epithelial cells and epidermal adnexa (such as hair follicles, hairs, sebaceous glands or sweat glands) were present in the specimen. An epidermoid tumor was diagnosed, if squamous stratified epithelium and desquamated epithelial cells were present without epidermal adnexa.

If only laminated keratin and desquamated epithelial cells but no squamous epithelium were present in the specimen, no diagnosis could be made based on the histopathological specimen. Therefore, these tumors were classified as desquamated epithelial cells without epithelial lining.

Patients were excluded whenever relevant clinical data were not available, or whenever a different tumor was retrospectively diagnosed after reevaluating available radiological, surgical and histopathological data.

The last outpatient visit to the neurologist was regarded as the last follow-up date. Data were analyzed using Microsoft Excell 2000 and SPSS 15.0 for Windows.

\section{Results}

\section{Demographics}

Initially, 23 patients with an intraspinal dermoid/epidermoid tumor were identified. However, five patients were subsequently excluded because of several reasons. In two patients the diagnosis of a teratoma was made and in three patients the tumor was extraspinally localized. The remaining 18 patients were included in the analysis. Nine patients were male and nine were female. Age at surgery ranged from eight months to 62 years (mean 13.3 years), including seven patients presenting under two years of age. Most tumors were located at lumbosacral level $(16=89 \%)$. Associated dysraphic anomalies were present in all but one patient ( Table 8.1). 
Intraspinal dermoid and epidermoid tumors

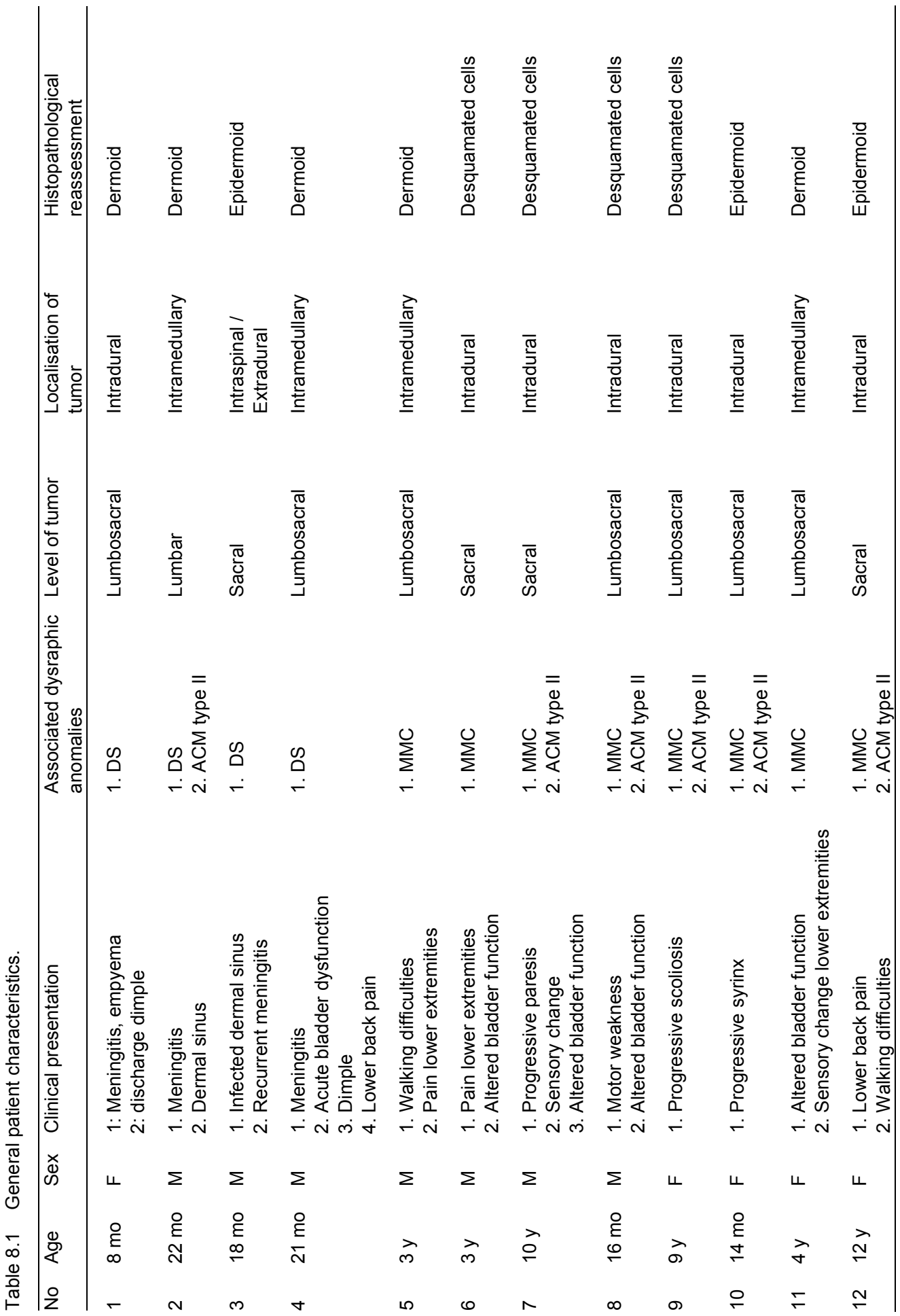




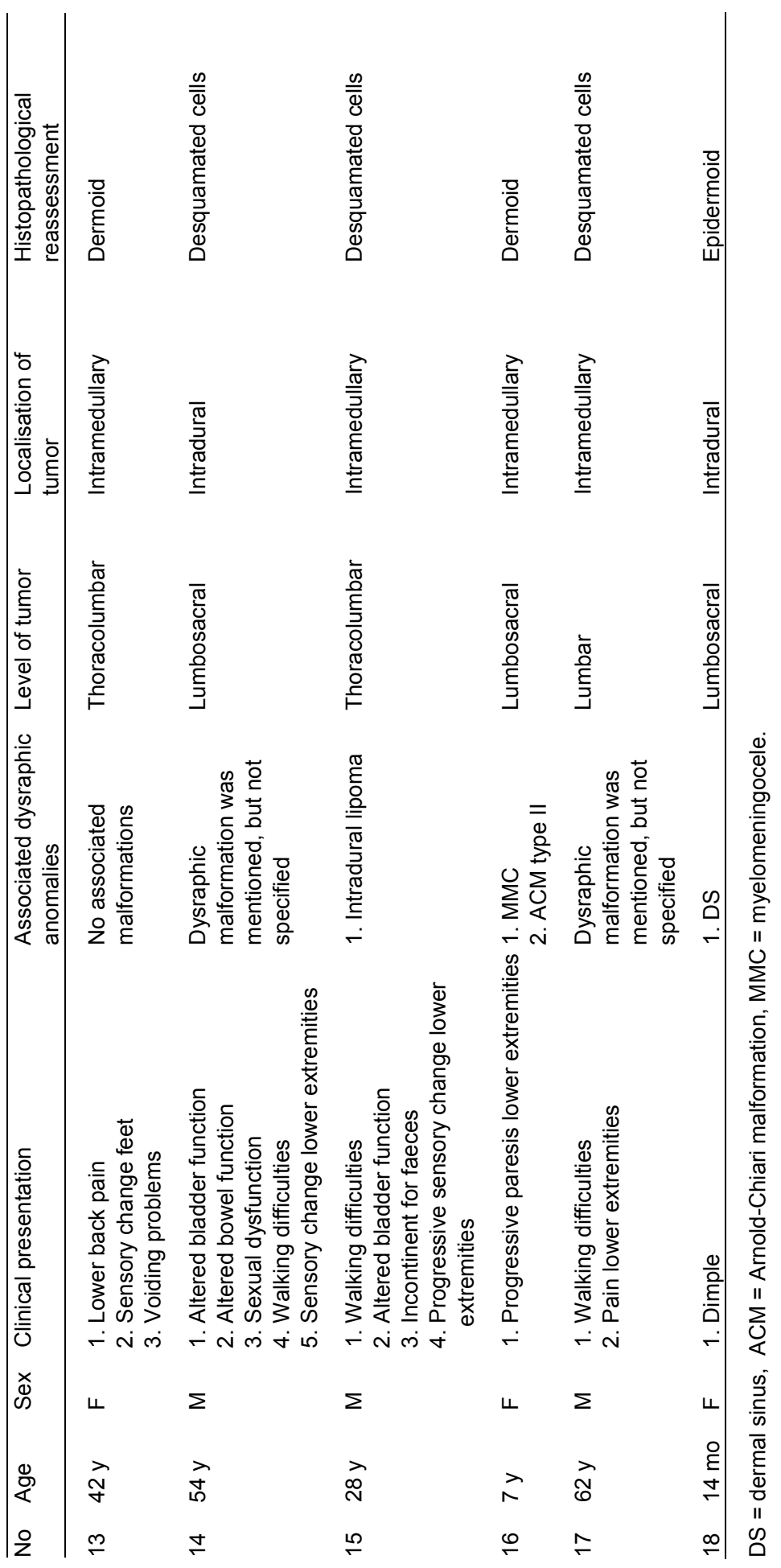




\section{Histopathological reassessment}

Seven dermoid and four epidermoid tumors were diagnosed by our neuropathologist. In the other seven specimens, only desquamated epithelial cells without epithelial lining were observed (Table 8.1).

\section{Surgical indication}

Because most patients presented with more than one symptom, in many cases the indication for surgical intervention was based on a combination of symptoms and signs, especially when they were progressive. We recorded all relevant symptoms and compared the pre- and postoperative state for each one of them.

In 18 cases, 41 relevant symptoms were reported (Table 8.2). Disturbances in gait and motor function were the most common indication $(22 \%)$ leading to surgical intervention, followed by urological problems (19.5\%) and pain $(14.6 \%)$. Four of 5 patients with a dermal sinus presented with an infection.

Table $8.2 \quad$ Signs and symptoms.

\begin{tabular}{lcc}
\hline Symptoms & Frequency & $\%$ \\
\hline Gait / motor & 9 & 22,0 \\
Urological problem & 8 & 19,5 \\
Pain & 6 & 14,6 \\
Dimple & 5 & 12,2 \\
Sensory disturbances & 4 & 9,8 \\
Meningitis / Empyema & 4 & 9,8 \\
Defecations & 2 & 4,9 \\
Sexual dysfunction & 1 & 2,4 \\
Scoliosis & 1 & 2,4 \\
Progressive syrinx & 1 & 2,4 \\
Total & 42 & 100,0 \\
\hline
\end{tabular}

\section{Imaging}

In retrospect, 17 intraspinal tumors could be evaluated on preoperative MR images (Figures 8.1, 8.2). In one patient, the tumor was obscured by a large abscess. Fifteen tumors were located at lumbosacral level, two at thoracic level.

On T1-weighted MR images, most dermoid and epidermoid tumors were isointens to the cerebrospinal fluid (CSF) or the spinal cord $(N=15 / 17)$, and two tumors (in both cases, only desquamated cells were seen after histopathological reassessment) were hyperintens to the spinal cord (Table 8.4). On T2-weighted images both dermoid and epidermoid tumors were isointens to CSF in most of the patients $(N=13 / 17)$. However, both tumors may have a heterogeneous signal intensity, intermediate between CSF and 
spinal cord as well $(\mathrm{N}=3 / 17)$. One lesion was isointens to the spinal cord on T2-weighted images, but in this case only desquamated cells were described after histopathological reexamination ( Table 8.4).

Interestingly, the radiological diagnosis, using the criteria as described in the literature ${ }^{1,4-6,8,18,32-34,53,54,57}$, was in accordance with histopathological diagnosis in only five patients. In seven patients a dermoid or epidermoid tumor was described on MR images, while histopathological re-examination revealed only desquamated cells without epithelial lining (Table 8.4).

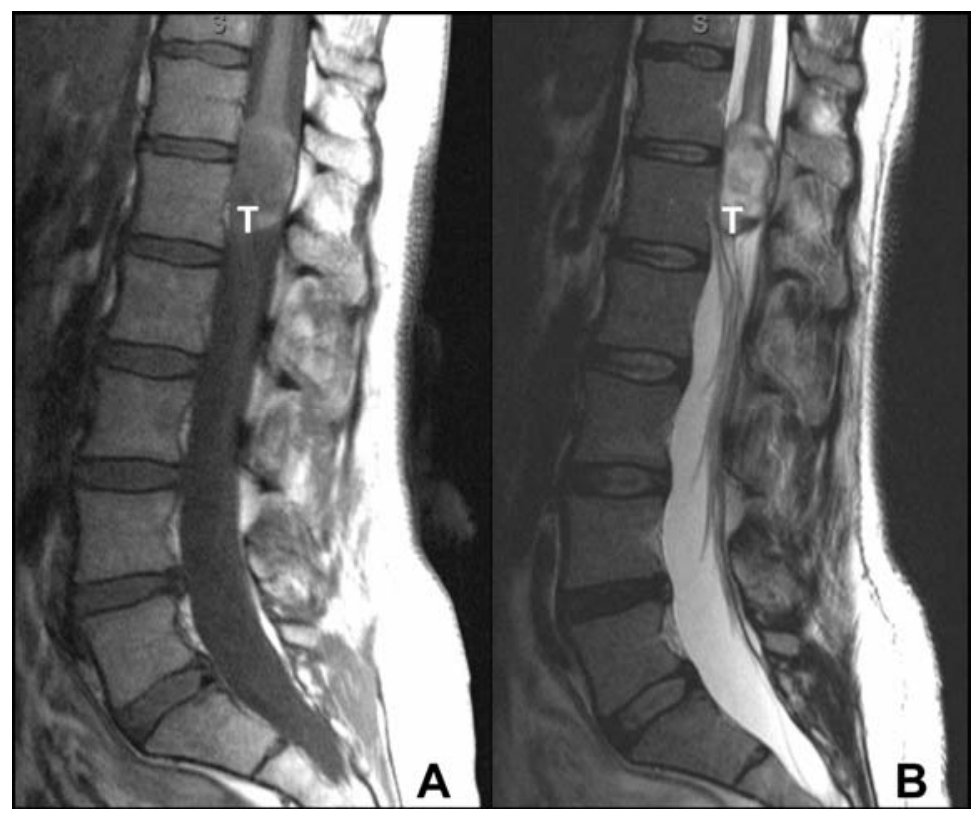

Figure 8.1 Sagittal MR images demonstrating an intramedullary dermoid tumor $(T)$ at Th12/L1 level.

A: T1-weighted MR image. Signal intensity of the lesion is isointense to the cord and heterogeneous.

B: T2-weighted MR image. Signal intensity of the lesion is isointense to the CSF and heterogeneous

\section{Intraoperative findings}

Eight tumors were located intramedullary, 9 extramedullary but intradurally, and one intraspinal but extradurally (Table 8.1). According to the operative notes, nine dermoid tumors including hairs were observed (Tables 8.3, 8.4). In addition, two tumors were named a dermoid, although no arguments for this conclusion were given. In three patients the tumor was macroscopically 
described as an epidermoid. In three patients, a tumor was mentioned but not specified and in one patient no tumor was mentioned at all.

The macroscopical aspect of the tumor, as observed by the surgeon, was in accordance with the histopathological reassessment in eight patients (Table 8.3, Figure 8.3).

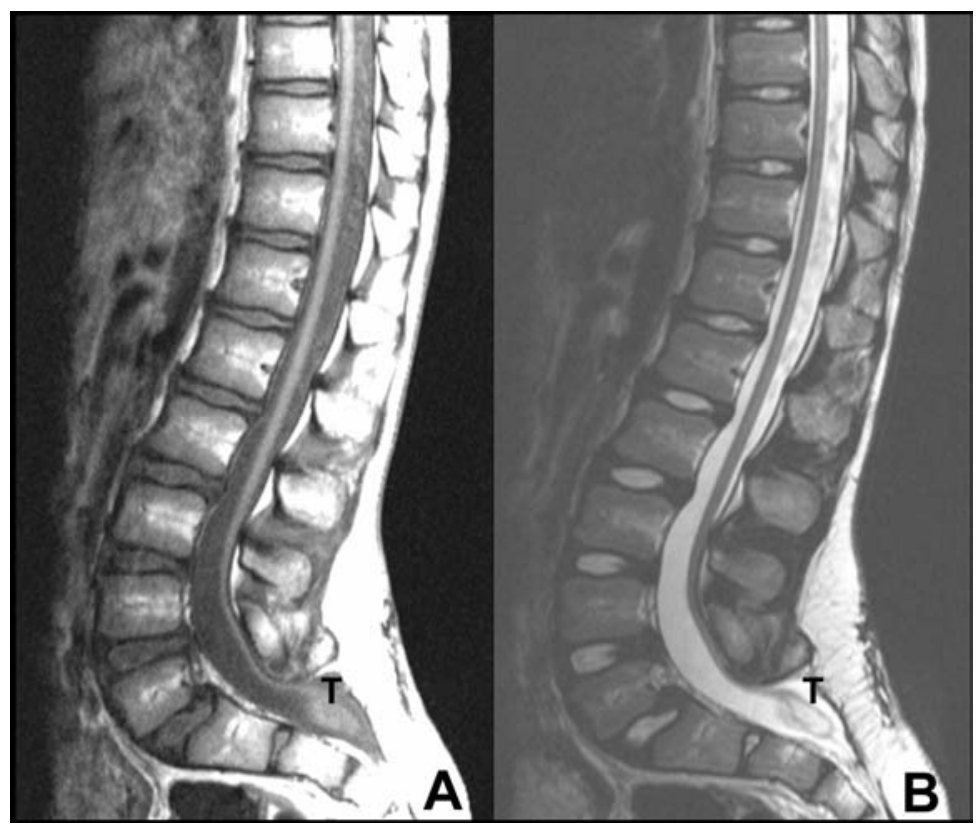

Figure 8.2 Sagittal MR image demonstrating an intradural epidermoid tumor (T) at sacral level in a patient with a previously closed lumbosacral myelomeningocele.

A: T1-weighted MR image. Signal intensity of the lesion is isointense to the cord and homogeneous.

B: T2-weighted MR image. Signal intensity of the lesion is isointense to the CSF and heterogeneous.

Table 8.3 Intraoperative diagnosis versus histopathological diagnosis.

\begin{tabular}{lcccc}
\hline Intraoperative diagnosis & \multicolumn{3}{c}{ Histopathological reassessment } & \\
& Epidermoid & Dermoid & Desquamated cells & Total \\
\hline Epidermoid & 2 & & 1 & 3 \\
Dermoid & 1 & & 1 & 2 \\
Dermoid including hairs & & 6 & 3 & 9 \\
Aspecific & 1 & 1 & 1 & 3 \\
No tumor observed during surgery & & & 1 & 1 \\
Total & 4 & 7 & 7 & 18 \\
\hline
\end{tabular}


In five patients, the surgeon observed either a dermoid or epidermoid tumor, but based on histopathological reassessment no final diagnosis could be made. If the surgeon reported an epidermoid, the pathologist did not in any case describe a dermoid and if the surgeon reported a dermoid, the pathologist did never describe an epidermoid.

According to the operative notes, the surgeon achieved macroscopical resection in five patients, incomplete in seven, and unspecified in five.

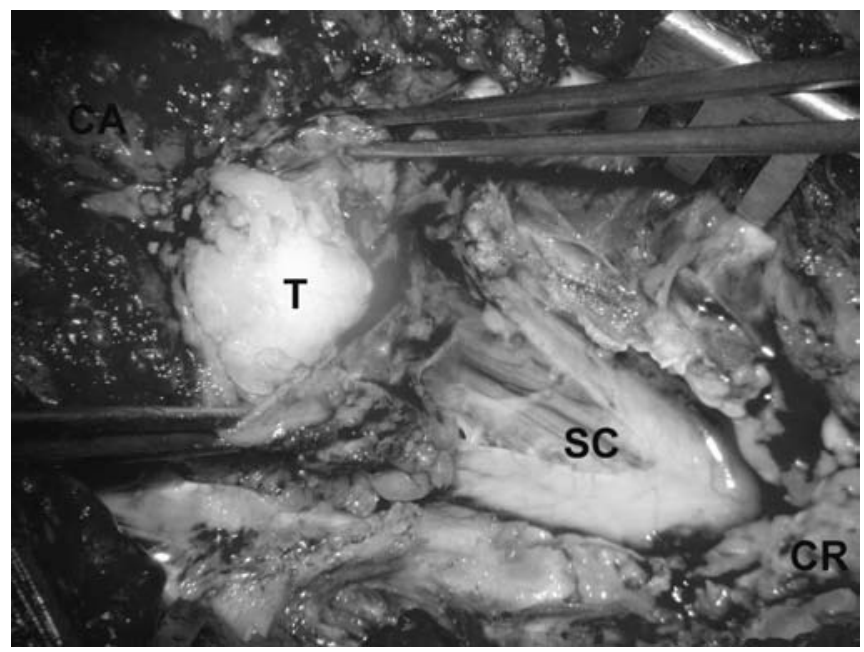

Figure 8.3 Intraoperative photograph of an intramedullary tumor at the level of the conus medullaris. In this case the radiological diagnosis was made of an epidermoid, whereas the surgeon reported a dermoid. Histopathological reassessment of the tumor specimen revealed only desquamated cells. $\mathrm{CR}=$ cranial, $\mathrm{CA}=$ caudal, $\mathrm{SC}=$ spinal cord, $T=$ tumor. See page 159 for colour figure.

\section{Patient outcome}

Follow-up ranged from 0 to 13 years (mean 4.5 years). One patient was operated very recently and has not attended the outpatient clinic for follow-up yet, whereas another patient was lost to follow-up. Postoperatively, $46 \%$ of preoperative symptoms improved, $34 \%$ were unchanged, $10 \%$ worsened and in the remaining $10 \%$ it was not or not yet known whether they improved, stabilized or worsened (Table 8.5). Gait and motor symptoms stabilized in two patients, improved in five and worsened in one. Urological problems stabilized in four patients, improved in two and worsened in two. Pain improved in four of six patients. Infection related to a dermal sinus tract was controlled in all patients, although resection of the tract was insufficient in one patient and two reexplorations had to be performed. Problems with respect to defecation, sensory disturbances and sexual disturbances did not improve postoperatively. 


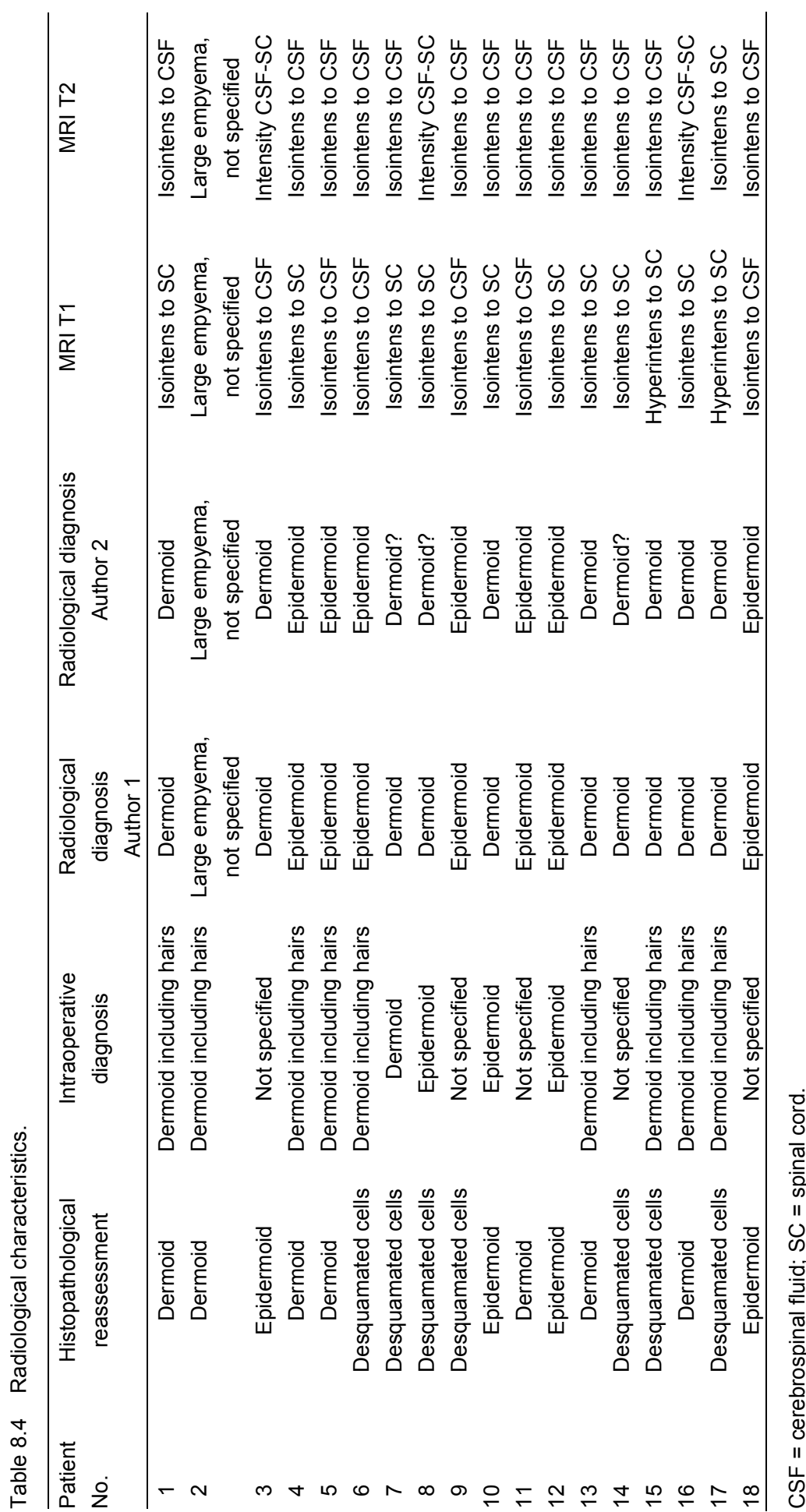


During follow-up, 3 patients presented with tumor recurrence, after 16 months to 6 years postoperatively. In all 3 cases a dermoid tumor was described intraoperatively, although this could not be confirmed by histopathological reassessment in one case. In all cases an intramedullary tumor at the level of the conus medullaris was described. A fourth patient was reoperated on twice because of a recurrent fistula after resection of an infected dermal sinus. Two tumor recurrences occurred despite the surgeon reporting complete resection during the initial operation. Symptoms leading to a second operation, stabilized in $50 \%$ and improved in $12,5 \%$.

Table $8.5 \quad$ Patient outcome.

\begin{tabular}{lccccc}
\hline Symptoms & Unchanged & Better & Worde & Unknown & Total \\
\hline Urological problem & 4 & 2 & 2 & & 8 \\
Defecation & 1 & & & 1 & 2 \\
Sexual dysfunction & 1 & & & 1 & 1 \\
Pain & 1 & 4 & & 1 & 9 \\
Gait / motor & 2 & 5 & 1 & 1 & 4 \\
Sensory disturbance & 2 & & 1 & & 1 \\
Scoliosis & 1 & & & & 1 \\
Progressive syrinx & 1 & & & & 4 \\
Dimple & 1 & 4 & & & 41 \\
Meningitis / Empyema & & 4 & & & \\
Total & 14 & 19 & 4 & & \\
\hline
\end{tabular}

\section{Discussion}

In this report, we describe a large cohort (the largest series reported to date) of patients with surgically treated intraspinal dermoid or epidermoid tumors. Clinical, radiological, intraoperative, and histopathological findings are analyzed. Although both tumor types have previously been described in case reports and even in some case series, there is no consensus on their etiology and nomenclature.

\section{Nomenclature}

In 1829, Cruveilhier described two lesions in the brain with the external surface similar to 'a pearl of the finest water' and named them 'pearly tumors' (from Sachs ${ }^{45}$ ). Since then, several other descriptive names were proposed, including cholesteatomas ${ }^{45}$, dermoids ${ }^{45}$ and epidermoids ${ }^{45}$.

Although Bostroem proposed to differentiate between dermoid and epidermoid tumors as early as 1897 , this did not gain recognition for a long time (Bostroem from Sachs ${ }^{45}$ ). In 1949, Sachs et al proposed to differentiate dermoid from epidermoid tumors by the presence of dermis and 'dermal glands ${ }^{\prime 45}$. 
Although it is currently known that hair follicles, sebaceous glands and sweat glands ('dermal glands') are in fact of epidermal origin ${ }^{13,24}$, this nomenclature is still in use and consequently dermoid and epidermoid tumors are still differentiated by their histological appearance. If the tumor does only contain squamous stratified epithelium and desquamated epithelial cells, an epidermoid tumor is diagnosed. If on the other hand additional adnexa (hair follicles and glands), are seen, a dermoid tumor is diagnosed.

\section{Histopathology of the dermoid tumor}

Dermoid tumors are well-defined, smooth, opaque, round or oval masses ${ }^{17,44}$. The cystic tumor contains thick, cheesy, yellowish material, in which hairs may be present ${ }^{2,17,44,58}$. Occasionally the contents may be yellow-brownish, mucous or even liquid ${ }^{2}$. Calcium deposits may be present in the cyst wall ${ }^{58}$.

The lining of dermoid cysts consists of stratified squamous epithelium supported by collagen, similar to epidermoid cysts. However, in the dermoid cyst wall, epidermal adnexa, such as hair follicles, sebaceous glands and sweat glands are present ${ }^{2,17,44,58}$ (Figure 8.4). The yellowish content of the dermoid cyst consists of laminated keratin, desquamated epithelium, but also includes sebum secreted by the sebaceous glands, hairs and other materials produced by the epidermal adnexa ${ }^{2,44}$. Blood vessels are detected in the connective tissue surrounding the tumor; they never penetrate the epithelial wall of the cyst ${ }^{2}$.

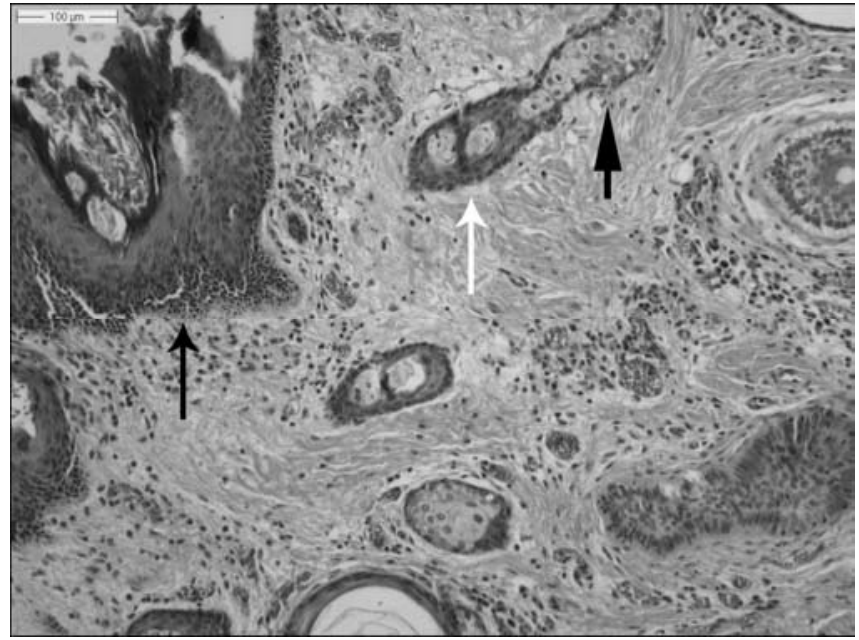

Figure 8.4 HE-staining. Photomicrograph of histopathology specimen showing a dermoid tumor. The wall of the tumor is lined by stratified squamous epithelium (black arrow). Hair follicles (white arrow) and sebaceous glands (arrow head) are present. See page 160 for colour figure. 


\section{Histopathology of the epidermoid tumor}

Macroscopically, epidermoid tumors are well-circumscribed, encapsulated lesions. Their whitish capsule has a mother-of-pearl sheen. Their surface may be smooth, nodular or lobulated ${ }^{17,44}$, whereas their interior is filled with soft, white material in concentric lamellae. Occasionally, the contents may be greybrownish and viscous ${ }^{17,33,44,58}$. Calcium deposits are rare ${ }^{17,44}$. The lining of epidermoid cysts consists of stratified squamous epithelium, supported by an outer layer of collagenous connective tissue (Figure 8.5). The epithelial lining reproduces the structure of normal epidermis ${ }^{17,44,58}$. Therefore, desquamation and breakdown of epithelial cells produces the lamellar character of the contents as well as a gradual expansion of the cyst ${ }^{17,44}$.

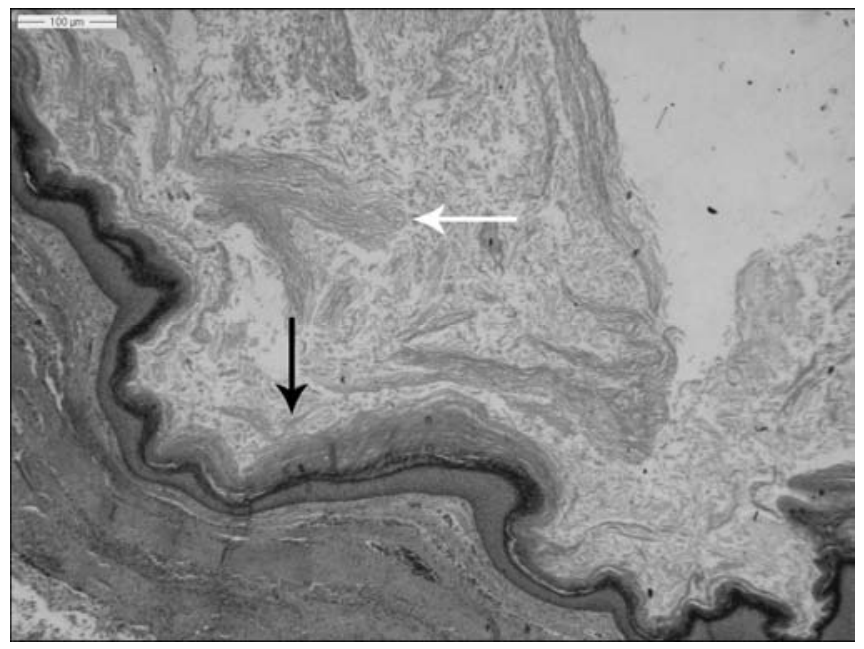

Figure 8.5 HE-staining. Photomicrograph of histopathology specimen showing an epidermoid tumor. The wall of the tumor is lined by stratified squamous epithelium (black arrow). The content of the tumor consists of laminated keratin and desquamated epithelial cells (white arrow). No epidermal adnexa are present.

See page 160 for colour figure.

\section{Etiology of the dermoid and epidermoid tumor}

Although it is known that spinal cutaneous inclusion tumors may develop after introducing cutaneous cells in the central nervous system ${ }^{16,56}$, as in case of a lumbar puncture or after closure of a dysraphic malformation, their etiology in case of congenital tumors is still unclear. Several theories exist on whether the displaced cutaneous cells develop into a dermoid or epidermoid tumor. For a long time, it was believed that if the inclusion of cutaneous ectodermal cells occurs early in embryonic life, the displaced cells are pluripotent and will develop into a dermoid tumor. If, on the other hand, the inclusion occurs later in 
fetal life, the cells have already undergone partial differentiation and will develop into an epidermoid tumor ${ }^{25,28,43,52}$. To date, this theory seems unlikely, since it is not until the end of the second month of embryonic life that the first hair follicles start developing from the cutaneous ectoderm ${ }^{24}$, whereas the neural tube closes already between the third and fifth week. Alternatively, some authors believed that a dermoid tumor might result from displacement of dermal and epidermal cells, whereas an epidermoid tumor might result from displaced epidermal cells only ${ }^{25,43}$. According to current embryological knowledge, this theory seems highly unlikely as well, as we now know that all components of dermoid tumors, including hair follicles, sebaceous glands and sweat glands, are in fact of epidermal instead of dermal origin ${ }^{13,24}$. It is well known from research in cell biology that embryonic development of epidermal adnexa in normal skin is induced by the underlying dermis ${ }^{7,13,24,38}$. Many regulatory factors are involved in this induction, such as wingless related (Wnt) signalling, lymphoid enhancer binding factor 1 (LEF1) and T-cell factor (TCF) proteins, fibroblast growth factors (FGFs) and bone morphogenetic proteins $(\mathrm{BMPs})^{13,14}$. Although not yet proven, the presence or absence of these regulatory factors may be an important determinant for an inclusion of cutaneous ectodermal cells to develop into a dermoid or epidermoid tumor.

Not only the dermis, but also other tissues develop from the mesoderm (subcutaneous fat, muscles, spinal arches, etc.). Additional involvement of the mesoderm in case of a spinal inclusion tumor may therefore not merely result in the development of a dermoid tumor, but instead give rise to a more complex intramedullary lipoma or split cord malformation.

\section{Clinical presentation}

Presenting signs and symptoms depend on the location and extent of the tumor. According to the literature, motor disturbances and pain are frequent ${ }^{23,27,52}$, the former presenting as flaccid or spastic motor weakness and gait disturbances ${ }^{52}$, the latter as pain in the back or radiating into the lower extremities. In children, pain may manifest itself through irritability or frequent crying, often exaggerated by coughing or sneezing ${ }^{33,52}$. Sensory abnormalities and sphincter or urological dysfunction are frequent as well ${ }^{2,3,23,27,48,52}$. In our patients, motor disturbances, urological dysfunction, and pain were the most frequently reported symptoms. Reflex abnormalities, spasms, musculoskeletal deformities (scoliosis, club foot) and sexual disturbances ${ }^{33,48,52}$ may complete the clinical picture. Finally, in patients with an associated dermal sinus tract, infection may be the presenting symptom, ranging from a local tract infection to a potentially lethal meningitis or an intraspinal empyema ${ }^{55}$. In our series, all patients with a dermal sinus presented with an infection. 


\section{Imaging}

In the literature, epidermoid tumors are reported as hypointens on T1-weighted MR images, similar to CSF and usually as hyperintens on T2-weighted images, again similar to $\operatorname{CSF}^{1,4-6,8,33,53,54,57}$. Dermoid tumors on the other hand are reported as usually hyperintens on T1-weighted images, due to the presence of fatty secretions of sebaceous glands and cholesterol, and are usually hypointens on T2-weighted images ${ }^{4-6,8,18,32-34,53,54,57}$. Importantly, signal intensity of both epidermoid and dermoid tumors may be homogeneous or heterogeneous ${ }^{1,6,32,39}$. Therefore, in spite of their general characteristics, both tumors may show a variety of signal intensities on T1- and T2-weighted MR images. In this regard, Gupta et al. compared the MR images of nine intraspinal dermoid and epidermoid tumors and concluded they could not differentiate between both entities as there seems to be considerable overlap in signal intensities ${ }^{20}$. This was confirmed by our study (to the best of our knowledge the largest study in the literature). We observed that even state-ofthe-art MR imaging does not allow differentiation between dermoid and epidermoid tumors. Nevertheless, MR imaging, is mandatory in the preoperative work-up for any dysraphic malformation, and in case of spinal dermoid or epidermoid tumors will provide details about the position of the tumor relative to the spinal cord, the position of the conus medullaris and the presence of associated congenital malformations.

\section{Intraoperative findings}

In 14 of our 18 cases an intraoperative diagnosis with regard to the (epi)dermoid tumor was described in the operative record, which was confirmed by histopathological reassessment in only eight cases. In one case, the intraoperative suggestion of a dermoid was made, but no arguments for this diagnosis were given and after histopathological reassessment the diagnosis of an epidermoid tumor was made. In five cases only desquamated epithelial cells were seen by our neuropathologist in the histopathological specimen. Possibly, for some reason these specimens did not include parts of the tumor capsula but solely debris from the centre of the tumor.

Removing the epithelial lining of the tumor may be difficult and more importantly may be quite dangerous in case of an intramedullary lesion, especially at the level of the fragile conus medullaris. Especially when a subtotal resection is performed, all excised material, and if possible parts of the cyst wall, should be sent to the neuropathologist, to increase the chance of obtaining a reliable diagnosis, since histopathological diagnosis still remains the gold standard. 


\section{Outcome}

Patient outcome in this series is encouraging, as $80 \%$ of patients had their neurological status improved or at least stabilized.

Four of 41 preoperative symptoms, recorded in three patients, worsened during follow-up. In one patient gait problems and sensory disturbances deteriorated after resection of a thoracic intramedullary tumor (only desquamated cells were seen at histopathological examination). Two other patients presented with urodynamic dysfunction, which gradually worsened after resection of a tumor (in both cases only desquamated cells were seen at histopathological examination) at the level of the conus medullaris. In these two patients this deterioration was possibly caused by surgical intervention, but because of the location of the primary developmental defect and accompanying tumor at the level of the conus medullaris, the reason for this deterioration remains subject of debate.

During follow-up, three patients presented with a tumor recurrence. In these patients, an intramedullary tumor had previously only partially been resected, because of its localisation at the level of the conus medullaris.

\section{General considerations}

The factors which cause the cutaneous ectoderm to interfere with the developing neural tube and subsequently produce these rare tumors remain obscure. Continued research on the development of the human skin and spinal cord, might elucidate these complex processes.

Despite the fact that the embryological mechanisms underlying the development of spinal dermoid and epidermoid tumors are interesting and not yet understood, it can be concluded from our results that the difference between intraspinal dermoid and epidermoid tumors is not important in clinical practice. Both tumors should be regarded as being part of a spectrum of spinal inclusion tumors, only to be differentiated by histopathological examination.

\section{Conclusions}

After reviewing 18 cases of surgically treated spinal dermoid and epidermoid tumors, we conclude that clinical outcome is encouraging, as $80 \%$ of patients had their neurological status improved or at least stabilized.

Clinical presentation, radiological characteristics, intraoperative findings and outcome do not differ between both tumor types. Therefore, the histopathological difference between intraspinal dermoid and epidermoid tumors is not important in clinical practice and should be avoided. Moreover, the name dermoid tumor should be regarded as a misnomer, because all of it's contents are in fact of epidermal origin. Both tumor types should be referred to as 'spinal cutaneous inclusion tumors'. 


\section{References}

1. Amato VG, Assietti R, Arienta C. Intramedullary epidermoid cyst: preoperative diagnosis and surgical management after MRI introduction. Case report and updating of the literature. J Neurosurg Sci 2002;46:122-126.

2. Arseni C, Dănăilă L, Constantinescu A, Carp N. Spinal dermoid tumours. Neurochirurgia (Stuttg) 1977;20:108-116.

3. Bailey IC. Dermoid tumors of the spinal cord. J Neurosurg 1970;33:676-681.

4. Barkovich AJ: Pediatric neuroimaging, ed 4th. Philadelphia, PA etc.: Lippincott Williams \& Wilkins, 2005.

5. Barkovich AJ, Edwards M, Cogen PH. MR evaluation of spinal dermal sinus tracts in children. AJNR Am J Neuroradiol 1991;12:123-129.

6. Bloomer CW, Ackerman A, Bhatia RG. Imaging for spine tumors and new applications. Top Magn Reson Imaging 2006;17:69-87.

7. Byrne C, Hardman M, Nield K. Covering the limb--formation of the integument. Journal of anatomy 2003;202:113-123.

8. Caro PA, Marks HG, Keret D, Kumar SJ, Guille JT. Intraspinal epidermoid tumors in children: problems in recognition and imaging techniques for diagnosis. J Pediatr Orthop 1991;11: 288-293.

9. Chadduck WM, Roloson GJ. Dermoid in the filum terminale of a newborn with myelomeningocele. Pediatr Neurosurg 1993;19:81-83.

10. Choremis C, Economos D, Gargoulas A, Papadatos C. Intraspinal epidermoid tumours (cholesteatomas) in patients treated for tuberculous meningitis. Lancet 1956;271:437-439.

11. Dias MS, McLone DG, Partington M. Normal and Abnormal Embryology the Spinal Cord and Spine, in Youmans, Winn HR, Kliot M, et al (eds): Youmans neurological surgery, ed 5th. Philadelphia, PA: Saunders, 2004;4:4239-4288.

12. French BN. The embryology of spinal dysraphism. Clin Neurosurg 1983;30:295-340.

13. Fuchs E. Scratching the surface of skin development. Nature 2007;445:834-842.

14. Fuchs E, Raghavan S. Getting under the skin of epidermal morphogenesis. Nature reviews. Genetics 2002;3:199-209.

15. Gandon Y, Hamon D, Carsin M, Guegan Y, Brassier G, Guy G, Husain A, Simon J. Radiological features of intradural epidermoid cysts. Contribution of MRI to the diagnosis. J Neuroradiol 1988;15:335-351.

16. Gibson T, Norris W. Skin fragments removed by injection needles. Lancet 1958;2:983-985.

17. Graham DI, Lantos PL, Greenfield JG. Greenfield's neuropathology. London: Arnold, 1997.

18. Graham DV, Tampieri D, Villemure JG. Intramedullary dermoid tumor diagnosed with the assistance of magnetic resonance imaging. Neurosurgery 1988;23:765-767.

19. Guidetti B, Gagliardi FM. Epidermoid and dermoid cysts. Clinical evaluation and late surgical results. J Neurosurg 1977;47:12-18.

20. Gupta S, Gupta RK, Gujral RB, Mittal P, Kuriyal M, Krishnani N. Signal intensity patterns in intraspinal dermoids and epidermoids on MR imaging. Clin Radiol 1993;48:405-413.

21. Herman JM, McLone DG, Storrs BB, Dauser RC. Analysis of 153 patients with myelomeningocele or spinal lipoma reoperated upon for a tethered cord. Presentation, management and outcome. Pediatr Neurosurg 1993;19:243-249.

22. Kukreja K, Manzano G, Ragheb J, Medina LS. Differentiation between pediatric spinal arachnoid and epidermoid-dermoid cysts: is diffusion-weighted MRI useful? Pediatr Radiol 2007;37:556-560.

23. Kumar S, Gulati DR, Mann KS. Intraspinal dermoids. Neurochirurgia (Stuttg) 1977;20: 105-108.

24. Larsen WJ, Sherman LS: Human embryology, ed 3rd. New York, N.Y., etc.: Churchill Livingstone, 2001.

25. List CF. Intraspinal epidermoids, dermoids, and dermal sinuses. Surg Gynecol Obstet 1941;73:525-538. 
26. Lombardi G, Passerini A. Congenital tumours of the spinal cord. Acta Radiol Diagn (Stockh) 1966;5:1047-1050.

27. Lunardi $P$, Missori $P$, Gagliardi FM, Fortuna A. Long-term results of the surgical treatment of spinal dermoid and epidermoid tumors. Neurosurgery 1989;25:860-864.

28. Manno NJ, Uihlein A, Kernohan JW. Intraspinal epidermoids. J Neurosurg 1962;19:754-765

29. Matsui H, Kanamori M, Yudoh K, Ohmori K, Yasuda T, Wakaki K. Cystic spinal cord tumors: magnetic resonance imaging correlated to histopathological findings. Neurosurg Rev 1998; 21:147-151.

30. Mazzola CA, Albright AL, Sutton LN, Tuite GF, Hamilton RL, Pollack IF. Dermoid inclusion cysts and early spinal cord tethering after fetal surgery for myelomeningocele. $\mathrm{N}$ Engl J Med 2002;347:256-259.

31. McDonald JV, Klump TE. Intraspinal epidermoid tumors caused by lumbar puncture. Arch Neurol 1986;43:936-939.

32. Mhatre $\mathrm{P}$, Hudgins $\mathrm{PA}$, Hunter $\mathrm{S}$. Dermoid cyst in the lumbosacral region: radiographic findings. AJR Am J Roentgenol 2000;174:874-875.

33. Myles ST, Hamilton MG: Intraspinal Congenital Cysts: Neurenteric, Arachnoid, Dermoid, and Epidermoid, in Albright AL, Pollack IF, Adelson PD (eds): Principles and Practice of Pediatric Neurosurgery, ed 2. New York, Stuttgard: Thieme, 2008.

34. Najjar MW, Kusske JA, Hasso AN. Dorsal intramedullary dermoids. Neurosurgical review 2005;28:320-325.

35. Nelson MD Jr, Bracchi M, Naidich TP, McLone DG. The natural history of repaired myelomeningocele. Radiographics 1988;8:695-706.

36. Ogden AT, Khandji AG, McCormick PC, Kaiser MG. Intramedullary inclusion cysts of the cervicothoracic junction. Report of two cases in adults and review of the literature. $\mathrm{J}$ Neurosurg Spine 2007;7:236-242.

37. Pansey BK, Verma A, Sood PK, Chabra SC, Pansey M. Dermoid tumours occurring at the site of previous meningocele repair. Clin Neurol Neurosurg 1988;90:137-140.

38. Paus R, Cotsarelis G. The biology of hair follicles. New England journal of medicine 1999; 341:491-497.

39. Penisson-Besnier I, Guy G, Gandon Y. Intramedullary epidermoid cyst evaluated by computed tomographic scan and magnetic resonance imaging: case report. Neurosurgery 1989;25:955-959.

40. Pennybacker J, Tytus JS. Pearly tumours in relation to the central nervous system. J Neurol Neurosurg Psychiatry 1956;19:241-259.

41. Per $\mathrm{H}$, Kumandaş $\mathrm{S}$, Gümüş $\mathrm{H}$, Yikilmaz $\mathrm{A}$, Kurtsoy $\mathrm{A}$. latrogenic epidermoid tumor: late complication of lumbar puncture. J Child Neurol 2007;22:332-336.

42. Potgieter S, Dimin S, Lagae L, Van Calenbergh F, Plets C, Demaerel P, Casaer P. Epidermoid tumours associated with lumbar punctures performed in early neonatal life. Dev Med Child Neurol 1998;40:266-269.

43. Rand RW, Rand CW. Intraspinal tumors of childhood. Springfield III: Charles C. Thomas, 1960.

44. Russell DS, Rubinstein LJ. Pathology of tumours of the nervous system. London, 1989.

45. Sachs E, Horrax G. A cervical and a lumbar pilonidal sinus communicating with intraspinal dermoids. J Neurosurg 1949;6:97-112.

46. Scott RM, Wolpert SM, Bartoshesky LE, Zimbler S, Klauber GT. Dermoid tumors occurring at the site of previous myelomeningocele repair. J Neurosurg 1986;65:779-783.

47. Shaywitz BA. Epidermoid spinal cord tumors and previou.s lumbar punctures. Journal of pediatrics 1972;80:638-640.

48. Shikata J, Yamamuro T, Mikawa Y, Kotoura Y. Intraspinal epidermoid and dermoid cysts. Surgical results of seven cases. Arch Orthop Trauma Surg 1988;107:105-109.

49. Steffey DJ, De Filipp GJ, Spera T, Gabrielsen TO. MR imaging of primary epidermoid tumors. J Comput Assist Tomogr 1988;12:438-440.

50. Storrs BB. Are dermoid and epidermoid tumors preventable complications of myelomeningocele repair? Pediatric neurosurgery 1944;20:160-162. 
51. Sundaram C, Paul TR, Raju BV, Ramakrishna Murthy T, Sinha AK, Prasad VS, Purohit AK. Cysts of the central nervous system: a clinicopathologic study of 145 cases. Neurol India 2001;49:237-242.

52. Takeuchi J, Ohta T, Kajikawa H. Congenital tumours of the spinal cord, in Vinken PJ, Bruyn GW (eds): Handbook of clinical neurology. Amsterdam: North-Holland publishing company, 1978;32:355-392.

53. Tang L, Cianfoni A, Imbesi SG. Diffusion-weighted imaging distinguishes recurrent epidermoid neoplasm from postoperative arachnoid cyst in the lumbosacral spine. J Comput Assist Tomogr 2006;30:507-509.

54. Teksam M, Casey SO, Michel E, Benson M, Truwit CL. Intraspinal epidermoid cyst: diffusionweighted MRI. Neuroradiology 2001;43:572-574.

55. van Aalst J, Beuls EA, Cornips EM, Vanormelingen L, Vandersteen M, Weber JW, Vles JS. Anatomy and surgery of the infected dermal sinus of the lower spine. Child's nervous system ChNS official journal of the International Society for Pediatric Neurosurgery 2006;22: 1307-1315.

56. Van Gilder JC, Schwartz HG. Growth of dermoids from skin implants to the nervous system and surrounding spaces of the newborn rat. J Neurosurg 1967;26:14-20.

57. Visciani A, Savoiardo M, Balestrini MR, Solero CL. latrogenic intraspinal epidermoid tumor: myelo-CT and MRI diagnosis. Neuroradiology 1989;31:273-275.

58. Wilkins RH, Odom GL: Spinal intradural cysts. Amsterdam, 1976.

59. Zavanone M, Guerra P, Rampini PM, Crotti F, Vaccari U. A cervico-dorsal intramedullary epidermoid cyst. Case report and review of the literature. J Neurosurg Sci 1991;35:111-115.

60. Ziv ET, Gordon McComb J, Krieger MD, Skaggs DL. latrogenic intraspinal epidermoid tumor: two cases and a review of the literature. Spine 2004;29:E15-18. 


\section{Chapter 9}

General discussion and conclusion 


\section{General discussion and conclusion}

In this thesis several congenital malformations associated with a tethered cord are studied. All malformations are part of the wide spectrum of congenital anomalies representing spina bifida.

Most types of spina bifida are treated by neurosurgeons around the world at a quite regular rate, and many case reports (and sometimes case series) have been published on these subjects. On the other hand, much is still unknown, and in most cases the etiology of the malformation remains obscure.

Therefore, the aim of this thesis has been to provide a detailed description of a number of congenital malformations from the spectrum of spina bifida associated with a tethered cord, focussing on clinical, anatomical, embryological, radiological and surgical characteristics.

In most myelomeningocele patients, clinical evidence of damage to the spinal cord is observed. Their clinical presentation dependents on the level and extend of the lesion, ranging from mild neurological deficit to severe (para)plegia.

In 1984, Brunelli et al. demonstrated the possibility of intrauterine closure of spina bifida defects in an experimental rabbit model ${ }^{4}$. Michejda ${ }^{26}$ developed a similar experimental model, using primate fetuses. All fetuses underwent a laminectomy in utero, but in some of the fetuses the defect was subsequently surgically repaired. At birth (cesarean section) the surgically repaired animals were neurologically normal while the untreated animals displayed severe paraplegia and incontinence. Apparently the exposed spinal cord of the untreated animals had been damaged during intrauterine follow-up. In 1990, Heffez et al. suggested that the spinal cord injury in myelomeningocele patients did not only result from a congenital myelodysplasia, but was caused by secondary damage due to exposure of the neural tube to the amniotic fluid as well ${ }^{14}$. They tested this hypothesis in an experimental animal model using rat pups and indeed observed secondary damage to the cord, which was explained as being caused by exposure to the toxic amniotic fluid. Additional studies in rat and pig siblings showed evidence of toxic as well as physical trauma possibly causing secondary damage to the spinal cord ${ }^{15}$. Several other authors developed animal models as well ${ }^{25,34}$ and concluded that secondary damage (toxic or traumatic) to the cord to be at least partly responsible for the severe neurological condition in myelomeningocele patients. McLone et al. ${ }^{24}$ did not agree with other authors and concluded from an experiment using fetal delayed splotch mice that no evidence was found of deterioration of the exposed neural tube during the gestational period of a mouse. 
Because of the encouraging findings observed in most animal studies, in 1997 and 1998 the first human fetuses underwent intrauterine closure of their myelomeningocele defect ${ }^{1,5}$.

Currently, a large multi-center trial (MOMS = Management of Myelomeningocele Study) is still collecting data to perform an analysis of 100 patients operated on in utero and compare these findings with outcome in 100 control patients, who will undergo postnatal closure of their myelomeningocele defect.

Preliminary results indicate that intrauterine surgery does not lead to improved sensorimotor function ${ }^{38}$, and no improvement in functional bladder outcome is observed $^{18}$. However, good effects are seen both on hindbrain herniation and the number of patients to be treated with ventriculoperitoneal shunting (which are both significantly reduced ${ }^{37,39,40}$ ).

In chapter 2 we described the anatomy of the spinal cord at 20-21 weeks of gestation, using high-field magnetic resonance imaging (HFMRI). Two embryos were studied; a 20-week-gestation-age fetus with a large myelomeningocele, and a healthy 21-week-gestation-age fetus. No neurulation defects were observed at the level of the myelomeningocele. This finding strengthens the hypothesis that at least in some cases of spina bifida the spinal cord initially is well developed but is damaged later on chemically and mechanically. However, even though we did not observe a neurulation defect, severe elongation and tapering of the tethered spinal cord was clearly demonstrated.

Intrauterine closure or covering of the myelomeningocele prevents further damage to the exposed cord, but it seems unlikely that this procedure either could restore the ascensus of the cord or prevent further deformation by elongation. This might explain the disencouraging sensorimotor function results of fetal surgery in myelomeningocele patients. Also, it would explain the good results of intrauterine surgery on hindleg function in most experimental animal models (except the experiment of McLone et al. ${ }^{24}$ ), because the defect in these experiments was artificially created at midgestation and did not include an experimental tethering.

The spinal congenital dermal sinus (DS) is a well known entity in the literature. Approximately 150 cases have been reported (personal observation). Of these, $60 \%$ presented with an infection (meningitis or intraspinal empyema), even followed by lethal outcome in some cases ${ }^{2,3,6,9,30,35}$.

In chapter 3 we described the infected DS of the lower spine. In this chapter, the need for a careful examination of the midline neuraxis during routine clinical evaluation of the newborn is once more emphasized.

Dimples are relatively common in the newborn. In 2000, Weprin and Oakes published a review on coccygeal pits and concluded from an extensive research of the literature and a retrospective review of 1000 patients treated at their institution that dimples located under the gluteal cleft are: relatively 
common (2-4\%) and do not seem to be associated with significant risk of spinal and intraspinal abnormalities ${ }^{42}$. However, a dimple located above the gluteal cleft, and especially is associated cutaneous changes (hypertrichosis, angiomata, etc.) are found, is suggestive of a DS. If this typical dimple is not found during neonatal examination, a DS can cause a devastating clinical picture in a previously healthy child. Especially in case of infection, it is crucial for the neurosurgeon to understand the pathological anatomy of the DS. From this study we learned that the DS has a complex anatomical configuration, with some characteristic features. We noted that an inward-directed dural sleeve accompanies the DS into the intradural space, while the arachnoidal membrane in fact remains intact, thus preventing cerebrospinal fluid (CSF) leakage through the DS orifice. Therefore, CSF leakage from a DS is very rare and if CSF is seen leaking from the orifice of the DS, the presence of additional hydrocephalus should be taken into consideration. Only few cases with spontaneous leakage of CSF through a DS are described in literature. Gupta et al. reported on a patient with CSF leakage following a meningitis in which a DS was found ${ }^{13}$. In this patient, severe hydrocephalus was present. Martinez-Lage described four cases of hydrocephalus in patients with a $\mathrm{DS}^{23}$. One of these patients presented with leakage from the DS orifice (following a purulent meningitis). In addition, Casani-Martinez described a patient with CSF leakage and purulent meningitis, but unfortunately in this report no details were provided regarding hydrocephalus ${ }^{7}$. Meningitis in DS patients is thought to develop as a result of necrosis and subsequent rupturing of the DS tract or associated dermoid/epidermoid tumor ${ }^{41}$. Following the spread of microorganisms or dermoid material into the subarachnoid space, a bacterial meningitis or chemicoleptomeningitis may develop resulting in a postmeningitis hydrocephalus. Due to the high CSF pressure and the damaged subarachnoidal space, leakage through the DS fistula takes place subsequently.

An anatomical continuum exists between DS and conus medullaris. Even without the presence of fibrotic scar tissue developing after an infection, it is notoriously difficult to find a cleavage plane between both. Regarding this issue, we described two landmarks; the lowest branching-off rootlets from the conus and, if present, the filum terminale. Additionally, in this chapter, the first case was reported of two parallel dermal sinuses at the same vertebral level.

The DS-like stalk is studied in chapter 4 . This malformation is easily mistaken for a DS, but in the cases we encountered, we noted significant differences. Therefore, we proposed to consider this malformation as a distinct entity. The most important difference was found at histopathological examination of the stalk. At macroscopical, intraoperative inspection, we already noted that these stalks did not represent the smooth, white tube that is typical of a DS, but instead consisted of fibrous tissue. Additionally, the DS-associated dural 
sleeve, as we described in chapter 3 , was directed in an opposite, ventrodorsal direction. Histopathological examination revealed solid stalks without a lumen, consisting of connective tissue, adipose tissue, muscle, some dural and a few nerve elements. We therefore concluded that the content of these stalks is of mesodermal origin. This is in contrast with the DS, which is composed of ectodermal derivatives. For that reason, we stated that the DS-like stalks have a different etiology compared to the DS. Moreover, and of clinical importance, the DS-like stalk does not present with an infection, because the stalk does not contain a lumen and is therefore not prone to infection. For the same reason, no dermoid/epidermoid tumors develop inside the tract.

Although both malformations (DS and DS-like stalk) may develop after a nondisjunction of neural- and surface ectoderm, the DS-like stalk represents a mesodermal structure, during embryological development being dragged toward the skin surface, pulling the dural sleeve with it. This is in contrast with the DS, in which it is believed that, after a nondisjunction, the surface ectoderm remains attached to the neural ectoderm and surrounding mesodermal tissues, and is dragged in a dorso-ventral direction, and as a consequence produces an inward directed dural sleeve ${ }^{41}$.

The pathological mechanism of nondisjunction is currently not understood. Although all authors studying the DS hypothesize that the DS results from this nondisjunction, this hypothesis was never before tested in an experimental setting. We hypothesized that local nonclosure of the neural tube might lead to a local nondisjunction. For that reason, we tested this hypothesis in an experimental study using 50 chick embryos (chapter 5), which indeed resulted in DS-like malformations in several embryos. From our experiments it can be concluded that formation of a DS-like anomaly can be successfully induced by experimental continuation of the connection between neural tube and surface ectoderm. This finding strengthens the hypothesis that a human DS arises after nondisjunction of neural tube and ectoderm. However, the cause of this nondisjunction remains unclear.

In some of the chick embryos, a split cord malformation (SCM) developed. This type of spina bifida has previously been studied by Pang et al. ${ }^{27,28}$, who distinguished two SCM types. A type I SCM was described as two hemicords, each one being surrounded by its own dural sac and separated by a firm, osteocartilaginous septum, whereas in a type II SCM the two hemicords are lying within a single dural sac, only being separated by a nonrigid fibrous septum $^{28}$.

In chapter 6 we described a patient in which an exceptional anatomical configuration of a SCM was observed. Characteristics of both subtypes of a SCM were noted in this patient. We stated that both types of SCM are part of a spectrum or range in which the level of pathological expression is dependent on the amount of meninx primitiva available in the split between both 
hemicords. We agree with Pang et al. that all cases of SCM are most probably caused by the presence of an additional neurenteric canal.

Whereas most types of spina bifida develop at every spinal level, the Currarino triad, being one of the syndromes caused by a pathological development of the caudal eminence, is a complex malformation restricted to the most caudal part of the spine. The Currarino triad is an autosomal dominant disorder linked to the $7 q 36$ region $^{19,22,31}$. Approximately 250 patients worldwide are known to have this malformation. The triad consists of a bony sacral defect, an anorectal malformation, and a presacral mass ${ }^{8,20}$. In most patients, this presacral mass is a teratoma, an anterior myelomeningocele, or a combination of both ${ }^{16,20,21,43}$. In chapter 7 we described 5 patients with the triad and discussed the need for neurosurgical intervention in those cases. We concluded that fistulas between the bowel and the intradural space have to be closed immediately. Release of a tethered cord is a subject of debate. We stated that the only indication for surgical untethering is a progressive tethered cord syndrome. Because constipation is present in virtually every patient with the Currarino triad, we do not consider this a reliable symptom of a tethered cord in these patients. Additionally, we found no evidence that an anterior myelomeningocele, if present, should be resected. Because malignant teratomas in patients with the Currarino triad have been reported, all solid tumors should be resected unless the diagnosis of a teratoma can be excluded.

Spina bifida is not infrequently associated with a spinal dermoid or epidermoid inclusion tumor. With regard to the etiology of these tumors, a distinction is made between spina bifida aperta and spina bifida occulta patients. If present in combination with spina bifida aperta, the tumor is thought to result from implantation of dermal/epidermal tissue into the subdural space during previous surgical closure of the myelomeningocele/meningocele defect ${ }^{17,29,33,36}$. If on the other hand a spina bifida occulta patient, who never before underwent surgery, presents with an inclusion tumor, this tumor most probably results from a cutaneous inclusion during embryological development.

In chapter 8 we presented the largest series of intraspinal dermoid and epidermoid tumors in the literature. We concluded that it is difficult, if not impossible to differentiate both tumor types solely by their clinical or radiological presentation and that histopathological examination remains the 'gold standard' when differentiating between both tumor types.

The intraoperative diagnosis of the surgeon proved to be the most reliable clinical parameter, but correlated in only $8 / 18$ cases with final histopathological examination. Although histopathological examination remains the 'gold standard', we concluded that the difference between both tumor types is not clinically relevant. Moreover, the term 'dermoid' is a misnomer, because all of its contents are in fact of epidermal/ectodermal origin. The subdivision 
dermoid-epidermoid is based on the wrong assumption that all dermal appendages develop from the dermis. We therefore stated that both tumor types should be referred to as 'spinal cutaneous inclusion tumors'.

\section{Future perspectives}

Detailed information with regard to clinical and surgical aspects in spina bifida malformations is crucial for the pediatric neurosurgeon. Much has been learned from previous studies, but on the other hand, many spina bifida-related problems remain unclear, or are at least subject of debate. In order to keep improving treatment of these patients, a continuous active approach to the problem called spina bifida is mandatory. All patients must undergo a systematic neurological examination by a child neurologist, as well as detailed radiological exams. Intraoperative anatomical details should be carefully described and if applicable, the specimen sent for histopathological examination should include a relevant part of the malformation.

Although neurosurgeons are primarily fascinated by clinical, anatomical, and surgical details, future experiments on the etiology of spina bifida should mainly focus on the genetics of the development of the human ectoderm, mesoderm, the process of disjunction, and the pathological process of nondisjunction. A better understanding of the genetic factors involved in these processes, might shed light on the obscure etiology of many spina bifida related malformations.

It should be taken into consideration as well that the embryological development of the central nervous system is closely related to the development of the skin. The mechanisms with reference to the development of the cutaneous ectoderm and the epidermis are still unclear. In recent studies $^{10-12}$, some of the processes concerning the development of the epidermis were unravelled, but experiments in the nearby future should focus on exploring this interesting subject to further elucidate the etiology of several complex neuro-developmental pathologies.

In 2007 Swedish scientists discovered the genetic failure giving rise to the DS in a special breed of dogs, the Rhodesian ridgeback ${ }^{32}$. These dogs, whose African origin dates centuries back, are characterized by a dorsal ridge of hair on their backs. This ridge of hair, for which evidently the dog breed is named, grows along its back in the opposite direction to the rest of its fur. The incidence of dermal sinuses is high $(8-10 \%)$ in the Rhodesian ridgeback. Both ridge and dermal sinuses are thought to result from a dominant mutation, involving duplication of three fibroblast growth factor (FGF) genes.

The correlation between hairs growing in the opposite direction and the development of dermal sinuses is not yet clear. We performed a DNA analysis (single nucleotide polymorphism analysis) on blood samples of some of our 
patients with a DS, to search for mutations in the FGF genes, but up until now, we were not able to confirm the presence of these mutations in our patients (personal observation). Nevertheless, in the nearby future other causative mutations might be found.

Apart from the promising progress in genetic knowledge of spina bifida related malformations, we should always try to put together all deformities as displayed by a spina bifida patient. This will lead to a better understanding of the different groups of malformations and syndromes, all belonging to the spectrum of spina bifida. The Currarino triad is an example of one of the multidisciplinary riddles that was solved, but many other syndromes are yet to be described and genetically evaluated. 


\section{References}

1. Adzick NS, Sutton LN, Crombleholme TM, Flake AW. Successful fetal surgery for spina bifida. Lancet 1998;352:1675-1676.

2. Altozano F. Un caso de sinus dermal congenito. Acta Pediatr Esp 1954;12:467-474.

3. Amador LV, Hankinson J, Bigler JA. Congenital spinal dermal sinuses. J Pediatr 1955;47: 300-310.

4. Brunelli G, Brunelli F. Experimental foetal microsurgery as related to myelomeningocele. Microsurgery 1984;5:24-29.

5. Bruner JP, Richards WO, Tulipan NB, Arney TL. Endoscopic coverage of fetal myelomeningocele in utero. Am J Obstet Gynecol 1999;180:153-158.

6. Cardell BS, Laurance B. Congenital dermal sinus associated with meningitis; report of a fatal case. Br Med J 1951;2:1558-1561.

7. Casani Martinez C, Otero Reigada MC, Perez Tamarit A, Alvarez Garijo JA, Asensi Botet F. [Lumbosacral dermal sinus as entry point of purulent meningitis. Report of 3 cases]. An Esp Pediatr 1991;34:68-70.

8. Currarino G, Coln D, Votteler T. Triad of anorectal, sacral, and presacral anomalies. AJR Am J Roentgenol 1981;137:395-398.

9. Dubreuilh MW. Abces Intra-medullaire consecutif a une tumeur dermoide congenitale. Bull Soc Anat Physiol Norm Pathol 1887;8:13-26.

10. Fuchs E. Beauty is skin deep: the fascinating biology of the epidermis and its appendages. Harvey Lect 1998;94:47-77.

11. Fuchs E. Scratching the surface of skin development. Nature 2007;445:834-842.

12. Fuchs E, Raghavan S. Getting under the skin of epidermal morphogenesis. Nat Rev Genet 2002;3:199-209.

13. Gupta DK, Shastank RR, Mahapatra AK. An unusual presentation of lumbosacral dermal sinus with CSF leak and meningitis. A case report and review of the literature. Pediatr Neurosurg 2005;41:98-101.

14. Heffez DS, Aryanpur J, Hutchins GM, Freeman JM. The paralysis associated with myelomeningocele: clinical and experimental data implicating a preventable spinal cord injury. Neurosurgery 1990;26:987-992.

15. Heffez DS, Aryanpur J, Rotellini NA, Hutchins GM, Freeman JM. Intrauterine repair of experimental surgically created dysraphism. Neurosurgery 1993;32:1005-1010.

16. Heij HA, Moorman-Voestermans CG, Vos A, Kneepkens CM. Triad of anorectal stenosis, sacral anomaly and presacral mass: a remediable cause of severe constipation. $\mathrm{Br} \mathrm{J}$ Surg 1990;77:102-104.

17. Herman JM, McLone DG, Storrs BB, Dauser RC. Analysis of 153 patients with myelomeningocele or spinal lipoma reoperated upon for a tethered cord. Presentation, management and outcome. Pediatr Neurosurg 1993;19:243-249.

18. Holzbeierlein J, Pope JI, Adams MC, Bruner J, Tulipan N, Brock JW, $3^{\text {rd }}$. The urodynamic profile of myelodysplasia in childhood with spinal closure during gestation. J Urol 2000; 164:1336-1339.

19. Kochling J, Karbasiyan M, Reis A. Spectrum of mutations and genotype-phenotype analysis in Currarino syndrome. Eur J Hum Genet 2001;9:599-605.

20. Kochling J, Pistor G, Marzhauser Brands S, Nasir R, Lanksch WR. The Currarino syndrome-hereditary transmitted syndrome of anorectal, sacral and presacral anomalies. Case report and review of the literature. Eur J Pediatr Surg 1996;6:114-119.

21. Lee SC, Chun YS, Jung SE, Park KW, Kim WK. Currarino triad: anorectal malformation, sacral bony abnormality, and presacral mass--a review of 11 cases. J Pediatr Surg 1997;32: 58-61.

22. Lynch SA, Bond PM, Copp AJ, Kirwan WO, Nour S, Balling R, Mariman E, Burn J, Strachan T. A gene for autosomal dominant sacral agenesis maps to the holoprosencephaly region at 7q36. Nat Genet 1995;11:93-95. 
23. Martinez-Lage JF, Perez-Espejo MA, Tortosa JG, Ros de San Pedro J, Ruiz-Espejo AM. Hydrocephalus in intraspinal dermoids and dermal sinuses: the spectrum of an uncommon association in children. Childs Nerv Syst 2006;22:698-703.

24. McLone DG, Dias MS, Goossens W, Knepper PA. Pathological changes in exposed neural tissue of fetal delayed splotch (Spd) mice. Childs Nerv Syst 1997;13:1-7.

25. Meuli M, Meuli-Simmen C, Hutchins GM, Yingling CD, Hoffman KM, Harrison MR, Adzick NS. In utero surgery rescues neurological function at birth in sheep with spina bifida. Nat Med 1995; 1:342-347.

26. Michejda M. Intrauterine treatment of spina bifida: primate model. Z Kinderchir 1984;39: 259-261.

27. Pang D. Split cord malformation: Part II: Clinical syndrome. Neurosurgery 1992;31:481-500.

28. Pang D, Dias MS, Ahab-Barmada M. Split cord malformation: Part I: A unified theory of embryogenesis for double spinal cord malformations. Neurosurgery 1992;31:451-480.

29. Pansey BK, Verma A, Sood PK, Chabra SC, Pansey M. Dermoid tumours occurring at the site of previous meningocele repair. Clin Neurol Neurosurg 1988;90:137-140.

30. Ripley W, Thompson DC. Pilonidal sinus as a route of infection in case of staphylococcus meningitis. Amer J Dis Child 1928;36:785-788.

31. Ross AJ, Ruiz-Perez V, Wang Y, Hagan DM, Scherer S, Lynch SA, Lindsay S, Custard E, Belloni E, Wilson DI, Wadey R, Goodman F, Orstavik KH, Monclair T, Robson S, Reardon W, Burn J, Scambler P, Strachan T. A homeobox gene, HLXB9, is the major locus for dominantly inherited sacral agenesis. Nat Genet 1998;20:358-361.

32. Salmon Hillbertz NH, Isaksson M, Karlsson EK, Hellmén E, Pielberg GR, Savolainen P, Wade $\mathrm{CM}$, von Euler H, Gustafson U, Hedhammar A, Nilsson M, Lindblad-Toh K, Andersson L, Andersson G. Duplication of FGF3, FGF4, FGF19 and ORAOV1 causes hair ridge and predisposition to dermoid sinus in Ridgeback dogs. Nat Genet 2007;39:1318-1320.

33. Scott RM, Wolpert SM, Bartoshesky LE, Zimbler S, Klauber GT. Dermoid tumors occurring at the site of previous myelomeningocele repair. J Neurosurg 1986;65:779-783.

34. Selcuki M, Manning S, Bernfield M. The curly tail mouse model of human neural tube defects demonstrates normal spinal cord differentiation at the level of the meningomyelocele: implications for fetal surgery. Childs Nerv Syst 2001;17:19-23.

35. Shenkin HA, Hunt AD, Horn RC. Sacrococcygeal sinus (pilonidal sinus) in direct continuity with the central canal of the spinal cord. Surg Gynecol Obstet 1944;79:655-659.

36. Storrs BB. Are dermoid and epidermoid tumors preventable complications of myelomeningocele repair? Pediatr Neurosurg 1994;20:160-162.

37. Sutton LN, Adzick NS, Johnson MP. Fetal surgery for myelomeningocele. Childs Nerv Syst 2003;19:587-591.

38. Tubbs RS, Chambers MR, Smyth MD, Bartolucci AA, Bruner JP, Tulipan N, Oakes WJ. Late gestational intrauterine myelomeningocele repair does not improve lower extremity function. Pediatr Neurosurg 2003;38:128-132.

39. Tulipan N. Intrauterine myelomeningocele repair. Clin Perinatol 2003;30:521-530.

40. Tulipan N, Sutton LN, Bruner JP, Cohen BM, Johnson M, Adzick NS. The effect of intrauterine myelomeningocele repair on the incidence of shunt-dependent hydrocephalus. Pediatr Neurosurg 2003;38:27-33.

41. van Aalst J, Beuls EA, Cornips EM, Vanormelingen L, Vandersteen M, Weber JW, Vles JS. Anatomy and surgery of the infected dermal sinus of the lower spine. Childs Nerv Syst 2006;22:1307-1315.

42. Weprin BE, Oakes WJ. Coccygeal pits. Pediatrics 2000;105:E69.

43. Yates VD, Wilroy RS, Whitington GL, Simmons JC. Anterior sacral defects: an autosomal dominantly inherited condition. J Pediatr 1983;102:239-242. 
Nederlandse samenvatting 


\section{Discussie en conclusie}

In dit proefschrift worden een aantal congenitale afwijkingen beschreven, die geassocieerd zijn met een gekluisterd ruggenmerg ('tethered cord'). Al deze afwijkingen zijn onderdeel van het brede spectrum van congenitale misvormingen die worden samengevat met de term spina bifida. Wereldwijd worden de meeste vormen van spina bifida regelmatig door neurochirurgen behandeld en in de literatuur zijn veel publicaties te vinden die, vanuit allerlei verschillende invalshoeken, dit onderwerp behandelen. Ondanks het feit dat er door de jaren heen steeds meer bekend is geworden over de patiënt met spina bifida, bestaan er nog steeds onduidelijkheden en is de etiologie van de misvorming in veel gevallen onbekend.

Het doel van dit proefschrift is geweest om een aantal vormen van een tethered cord bij spina bifida gedetailleerd te beschrijven met aandacht voor de pathologische embryologie, de (chirurgische) anatomie en de klinische en radiologische presentatie.

Bij veel patiënten met een meningomyelocele is er sprake van een klinisch evidente beschadiging van het ruggenmerg. De klinische presentatie van de patiënt is afhankelijk van het niveau en de uitgebreidheid van de laesie en varieert van milde neurologische symptomatologie tot ernstige paraplegie.

In 1984 demonstreerde Brunelli et al. ${ }^{4}$ in een proefdiermodel bij konijnen dat het mogelijk is om een spina bifida defect al in utero te sluiten. Michejda ${ }^{26}$ ontwikkelde een soortgelijk diermodel, waarbij primaten werden gebruikt. Alle foetussen in dit experiment ondergingen een laminectomie, waarna later bij sommige foetussen dit defect chirurgisch werd gesloten. Beide ingrepen vonden plaats in utero. Na geboorte (via een keizersnede) bleek dat de chirurgisch herstelde foetussen neurologisch normaal waren, terwijl er in de andere groep ernstige paraparese en incontinentie werd vastgesteld. Blijkbaar was het ruggenmerg van de niet chirurgisch herstelde dieren beschadigd tijdens de verdere intra-uteriene ontwikkeling.

In 1990 suggereerden Heffez et al. ${ }^{14}$ dat het ruggenmergsletsel in meningomyelocele patiënten waarschijnlijk niet alleen het resultaat is van een congenitale myelodysplasie, maar dat tevens secundaire schade wordt veroorzaakt door blootstelling van het ruggenmerg aan het amnionvocht. Deze hypothese werd getest in een experimenteel diermodel waarbij rattenpups werden gebruikt. De uitkomst van dit experiment was inderdaad dat er secundaire schade optrad, vermoedelijk ten gevolge van de toxiciteit van het amnionvocht. Ook uit andere experimenten met soortgelijke diermodellen ${ }^{15,25,34}$ werd geconcludeerd dat secundaire (toxische of mechanische) schade in ieder geval gedeeltelijk verantwoordelijk moet zijn voor de ernstige neurologische uitval in meningomyelocele patiënten. 
Er was echter ook weerstand tegen deze hypothese. McLone et al. ${ }^{24}$ beschreven een dierexperimenteel muizenmodel, waarin geen aanwijzingen werden gezien voor deze secundaire schade.

Vanwege de bemoedigende resultaten uit de meeste dierexperimenten ondergingen in 1997 en 1998 de eerste menselijke foetussen met een meningomyelocele een intra-uteriene sluiting van het meningomyelocele defect $^{1,5}$. Op dit moment worden er in een grote studie (MOMS = Management of Myelomeningocele Study) data verzameld vanuit meerdere instituten om 100 in utero geopereerde patiënten te vergelijken met 100 patiënten bij wie het meningomyelocele defect postnataal werd gesloten.

Voorlopige resultaten van deze trial laten zien dat intra-uteriene chirurgie geen effect heeft op uitval van sensibiliteit of motoriek ${ }^{38}$. Ook wordt geen verbetering gezien van de blaasfunctie ${ }^{18}$. Het aantal patiënten dat een Arnold-Chiari malformatie ontwikkelt lijkt echter significant te worden gereduceerd, net als het aantal patiënten dat behandeld moet worden vanwege hydrocefalus $\mathrm{s}^{37,39,40}$.

In hoofdstuk 2 van dit proefschrift wordt, middels gebruik van hoogveldsterkte MRI (HVMRI) de anatomie van het foetale ruggenmerg beschreven 20-21 weken postgestatie. Twee embryo's werden geanalyseerd en vergeleken; een foetus met een grote meningomyelocele ( 20 weken postgestatie) en een gezonde foetus ( 21 weken postgestatie).

Ter hoogte van de meningomyelocele werden geen aanwijzingen gezien voor een neurulatiedefect. Dit is in overeenstemming met de hypothese dat het ruggenmerg, ook in geval van een ernstige meningomyelocele, in eerste instantie normaal is aangelegd en secundair wordt beschadigd. Er werd echter wel een tethering en uitrekking gezien van het ruggenmerg. Het in utero bedekken of sluiten van het meningomyelocele defect voorkomt secundaire schade aan het ruggenmerg, maar het lijkt onwaarschijnlijk dat hierdoor de tethering en uitrekking van het ruggenmerg worden voorkomen. Dit kan het feit verklaren dat de resultaten van intra-uteriene chirurgie wat betreft neurologische uitval tot nu toe teleurstellend zijn. Ook zou dit een verklaring zijn voor de goede resultaten op beenfunctie die werden gezien na dierexperimenteel onderzoek. De chirurgische defecten in deze proefdieren werden immers pas aangebracht nadat er al een normale ontwikkeling van het ruggenmerg aan was vooraf gegaan.

De dermale sinus (DS) is een zeldzame, maar bekende afwijking. Tot nu toe zijn er ongeveer 150 patiënten beschreven in de literatuur (persoonlijk observatie). Van deze patiënten presenteert $60 \%$ zich met een infectie in de vorm van een meningitis of intraspinaal empyeem, soms zelfs met dodelijke afloop ${ }^{2,3,6,9,30,35}$.

In hoofdstuk 3 wordt de geïnfecteerde lumbosacrale DS beschreven. Het belang van goed klinisch onderzoek van de rug van een pasgeborene direct 
postpartum wordt hier nogmaals benadrukt. Lumbosacrale dimpels komen vrij vaak voor. Uit onderzoek blijkt dat een dimpel in of onder de bilnaad vaak voorkomt en niet moet worden geassocieerd met spinale of intraspinale afwijkingen ${ }^{42}$. Is de dimpel echter boven de bilnaad gelokaliseerd en is er tevens sprake van lokale huidafwijkingen, is de kans op een DS veel groter. Een DS die gemist wordt tijdens het postpartum onderzoek van de pasgeborene, kan later een dramatisch klinisch beeld veroorzaken. Kennis van de pathologische anatomie van deze afwijking is dan van essentieel belang voor de geconsulteerde neurochirurg.

Hoewel de anatomie van een DS complex is, konden we een aantal typische kenmerken beschrijven. De dura omgeeft de DS met een soort mouw richting het spinale kanaal. Daarbij blijft de spinale arachnoidea intact en lekkage van liquor door een DS is dan ook zeer zeldzaam. Is er toch sprake van liquorlekkage uit de opening van een DS, moet ernstig rekening worden gehouden met hydrocefalus ${ }^{7,13,23}$.

Indien er meningitis optreedt bij een patiënt met een DS, is dit waarschijnlijk het gevolg van het ruptureren van de locaal ontstoken en genecrotiseerde fistelgang ${ }^{41}$ of geassocieerde inclusietumor. Aangezien er nu een port d'entrée is ontstaan naar de subarachnoidale ruimte, kan er een bacteriële meningitis ontstaan. In geval van een geruptureerde inclusietumor kan dit aanleiding geven tot een chemicoleptomeningitis. Beide vormen kunnen weer leiden tot een postmeningitis hydrocefalus, die vanwege de hoge liquordruk en beschadigde arachnoidea lekkage van liquor uit een DS kan veroorzaken.

Tussen de DS en de conus medullaris is in veel gevallen geen scheidingsvlak waar te nemen. Zelfs zonder dat het operatiegebied is aangetast door een eerder doorgemaakte infectie, kan het zeer moeilijk zijn om dit scheidingsvlak te vinden. We beschreven twee anatomische 'landmarks' die kunnen helpen om het niveau van de conus te identificeren; de laagst aftakkende radices en het filum terminale. Tevens beschreven we in dit hoofdstuk het eerste geval van twee parallelle dermale sinus op eenzelfde spinaal niveau.

In hoofdstuk 4 wordt een variant van de DS beschreven, die we de DS-like stalk hebben genoemd. Dit kan worden vertaald als de 'DS-achtige streng'. Deze afwijking lijkt erg op een DS, maar toch beschreven we een aantal essentiële verschillen. Tijdens macroscopisch onderzoek van de strengen viel reeds op dat deze niet het gladde witglanzende aspect hadden zoals vaak wordt gezien bij een DS, maar er meer fibreus uitzagen. Ook bleek dat de durale mouw, zoals we die beschreven in hoofdstuk 3, niet richting het spinale kanaal gericht was, maar in een tegengestelde (ventrodorsale) richting. Het meest belangrijke verschil werd echter gezien na histopathologisch onderzoek. De streng bleek geen lumen te bevatten en was opgebouwd uit bindweefsel, vet, spier, sporen van dura en zelfs een paar neurale elementen. In tegenstelling tot de DS, die is opgebouwd uit ectodermale componenten, 
bestaat de DS-like stalk dus met name uit mesodermale derivaten. Daaruit concludeerden we dat er ook een verschil moet zijn in de etiologie van beide afwijkingen.

Het klinisch belang van deze bevindingen is dat een DS-like stalk, aangezien deze geen lumen bevat, geen aanleiding zal geven tot een infectie. Ook zal er geen cutane inclusietumor ontstaan.

Beide afwijkingen (DS en DS-like stalk) ontstaan waarschijnlijk als gevolg van een nondisjunctie tussen neuraal- en oppervlakte ectoderm. In het geval van een DS-like stalk is er sprake van een mesodermale structuur, die tijdens de embryologische ontwikkeling richting het huidoppervlak wordt meegesleept, waarbij de durale mouw zich in dezelfde richting ontwikkelt. Dit in tegenstelling tot de DS waarbij gedacht wordt dat na de nondisjunctie, het oppervlakte ectoderm niet loslaat van het neurale ectoderm en het omgevende mesoderm en in een dorsoventrale richting wordt meegesleept richting het spinale kanaal. In dit geval resulteert dit in een inwaarts gerichte durale mouw.

Het eerder genoemde mechanisme van nondisjunctie is tot op heden niet bekend. Hoewel er in de literatuur consensus bestaat over het feit dat dit mechanisme verantwoordelijk moet zijn voor het ontstaan van een DS, werd dit nooit eerder experimenteel getoetst. Om die reden ontwikkelden wij een experimenteel diermodel waarbij onze hypothese was dat een lokaal sluitingsprobleem van de neurale buis zou kunnen leiden tot een lokale nondisjunctie. Deze hypothese werd getest in een experimenteel diermodel, waarbij gebruik werd gemaakt van 50 in ovo geopereerde kippenembryo's. Dit experiment, dat inderdaad resulteerde in afwijkingen die sterke overeenkomsten vertoonden met een DS, wordt beschreven in hoofdstuk 5 . We concludeerden dat de ontwikkeling van een DS kan worden nagebootst middels een experimentele verlenging van de verbinding tussen de neurale buis en het oppervlakte ectoderm. Deze bevinding steunt de hypothese dat ook de menselijke DS het resultaat is van een nondisjunctie tussen de neurale buis en het oppervlakte ectoderm. Het blijft echter onduidelijk waarom deze nondisjunctie optreedt.

Bij sommige kippenembryo's werd gedurende het hierboven beschreven experiment een split cord malformation (SCM) vastgesteld. Deze term kan worden vertaald als gespleten of verdubbeld ruggenmerg. De SCM is uitgebreid bestudeerd door Pang et al. ${ }^{27,28}$, die twee vormen van SCM beschreef. Bij een SCM type I is het ruggenmerg gespleten, waarbij iedere helft is omgeven door een eigen dura. Tussen beide duraalzakken bevindt zich een stevig osteofibreus septum. In geval van een SCM type II liggen beide helften samen in een duraalzak en zijn slechts van elkaar gescheiden door een dunwandig, vliezig septum.

In hoofdstuk 6 beschreven we een patiënt met een bijzondere variant van een SCM. In dit geval was er sprake van een misvorming met kenmerken van 
zowel een type I als een type II SCM. We concludeerden dat er geen goed onderscheid kan worden gemaakt tussen beide types en dat deze beiden onderdeel zijn van een spectrum van afwijkingen. De hoeveelheid meninx primitiva die tussen beide ruggenmergshelften aanwezig is, is bepalend voor de mate van ernst waarin de SCM tot uiting komt. Zoals ook eerder door Pang et al. werd gesuggereerd, worden alle vormen van SCM meest waarschijnlijk veroorzaakt door de aanwezigheid van een extra neurenterisch kanaal.

De meeste vormen van spina bifida kunnen op ieder spinaal niveau voorkomen. Er zijn echter ook vormen die een bepaalde voorkeurslokalisatie hebben. De trias van Currarino is een syndroom dat veroorzaakt wordt door een stoornis in de aanleg van de caudale eminentie. Het is dan ook niet verwonderlijk dat dit syndroom zich beperkt tot het meest caudale gedeelte van de wervelkolom. De trias van Currarino is een autosomaal dominante aandoening die verbonden is aan de $7 q 36$ regio $^{19,22,31}$. Wereldwijd zijn er ongeveer 250 patiënten bekend met dit syndroom, dat bestaat uit een benig defect in het sacrum, een anorectale misvorming en een presacrale tumor ${ }^{8,20}$. In de meeste gevallen is deze presacrale tumor een teratoom, een anterieure meningomyelocele, of een combinatie van beiden ${ }^{16,20,21,43}$.

In hoofdstuk 7 beschreven we vijf patiënten met de trias, waarbij de nadruk is gelegd op de neurochirurgische behandeling. We concludeerden dat fistels tussen het colon en de intradurale ruimte onmiddellijk dienen te worden gesloten. Het blijft een onderwerp van discussie of het tethered cord moet worden geopereerd. De enige indicatie hiervoor is een progressief tethered cord syndroom. Omdat obstipatie in vrijwel iedere patiënt met de trias aanwezig is, kan dit niet worden beschouwd als een betrouwbare manifestatie van een tethered cord. We hebben geen bewijs gevonden voor het feit dat een eventueel aanwezige anterieure meningomyelocele operatief moet worden verwijderd. Omdat er in de literatuur melding wordt gemaakt van maligne teratomen bij patiënten met de trias, dienen alle vaste tumoren te worden verwijderd, tenzij de diagnose van een teratoom kan worden uitgesloten.

Inclusietumoren zijn niet ongewoon in combinatie met spina bifida. In de meeste gevallen betreft het hier dermoiden, epidermoiden of lipomen. De etiologie van intraspinale dermoiden en epidermoiden lijkt verschillend te zijn naar gelang er sprake is van spina bifida aperta of van spina bifida occulta. In geval van spina bifida aperta, wordt verondersteld dat deze tumoren ontstaan als gevolg van het achterblijven van huidresten in de subdurale ruimte tijdens het sluiten van het meningomyelocele/meningocele defect ${ }^{17,29,33,36}$. In spina bifida occulta patiënten, die nooit geopereerd zijn, ontstaan deze inclusietumoren meest waarschijnlijk als gevolg van een cutane inclusie tijdens de verstoorde embryologische ontwikkeling. 
In hoofdstuk 8 beschreven we de intraspinale dermoiden en epidermoiden. Het betreft hier de grootste serie tot nu toe in de literatuur. We concludeerden dat het vrijwel onmogelijk is om een onderscheid te maken tussen een dermoid en een epidermoid op basis van klinische of radiologische presentatie. Histopathologisch onderzoek is en blijft de gouden standaard.

Hoewel de peroperatieve inschatting van de neurochirurg de meest betrouwbare klinische parameter bleek wanneer het gaat om de differentiatie tussen beide tumorsoorten, bleek ook dat slechts in $8 / 18$ gevallen overeen te komen met de uiteindelijke histopathologische diagnose.

Het verschil tussen het intraspinale dermoid en epidermoid is daarom niet klinisch relevant. Bovendien is de term 'dermoid' een foutieve benaming, aangezien deze tumoren een epidermale/ectodermale oorsprong hebben. Het onder-scheid tussen dermoid en epidermoid is gebaseerd op de onjuiste aanname dat alle huidadnexen (haren, zweet- en talgklieren) zich ontwikkelen uit de dermis. We concludeerden dan ook dat beide tumorsoorten kunnen worden samengevat onder de naam 'spinale cutane inclusietumoren'.

\section{Aanbevelingen}

Er wordt steeds meer bekend over spina bifida. Toch blijven er nog veel vragen onbeantwoord, of in ieder geval onderwerp van discussie. Een continue actieve houding ten aanzien van deze patiëntengroep is dan ook gerechtvaardigd. Alle patiënten moeten systematisch worden onderzocht door een ervaren kinderneuroloog en goede radiologische beeldvorming is een vereiste. De neurochirurg moet oog hebben voor peroperatieve details en indien er weefsel voor histopathologisch onderzoek wordt ingestuurd, dient dit een relevant gedeelte van de afwijking te bevatten.

Hoewel neurochirurgen met name geïnteresseerd zijn in klinische presentatie, anatomie en chirurgische details, zal het toekomstig wetenschappelijk onderzoek naar spina bifida zich vooral moeten richten op de genetische ontwikkeling van het ectoderm, het mesoderm, het proces dat disjunctie wordt genoemd en het pathologische mechanisme achter nondisjunctie.

Een beter begrip van deze processen zal van belang zijn bij het ontrafelen van de factoren die een rol spelen bij de etiologie van veel aan spina bifida gerelateerde afwijkingen.

De ontwikkeling van het centraal zenuwstelsel hangt sterk samen met de ontwikkeling van de huid. Wat betreft de kennis van de ontwikkeling van de huid, en dan met name de ontwikkeling van de epidermis, is er de laatste jaren vooruitgang geboekt ${ }^{10,11,12}$. In toekomstige experimenten die zich richten op de etiologie van spina bifida, zullen deze dermatologische ontwikkelingen zeker betrokken moeten worden. 
In 2007 ontdekten Zweedse wetenschappers het genetische defect dat een DS veroorzaakt in de Rhodesian ridgeback, een hondenras ${ }^{32}$. Deze honden, die afstammen van een zeer oud Afrikaans hondenras hebben een typerende richel haar op de middellijn van hun rug. In deze richel groeien de haren in tegenovergestelde richting ten opzichte van de haren in de rest van de vacht. Bij de Rhodesian ridgebacks wordt er in $8-10 \%$ een DS vastgesteld. Zowel de afwijkende haargroei in de middellijn, als ook de DS zijn vermoedelijk het resultaat van een dominante mutatie, waarbij de duplicatie van het genetisch materiaal van drie fibroblast groeifactoren (FGF) een belangrijke rol speelt.

The relatie tussen haren die een andere groeirichting hebben en het ontwikkelen van een DS is nog niet duidelijk. Bij een aantal van onze patiënten met een DS hebben we DNA onderzoek (single nucleotide polymorphism analyse) op bloedmonsters verricht, met specifieke aandacht voor de genen die betrokken zijn bij de expressie van FGF. Tot nu toe konden we de defecten, zoals aangetoond bij de Rhodesian ridgeback, echter niet aantonen bij de mens.

Los van de vooruitgang in het genetisch onderzoek, die veelbelovend is, blijft het erg belangrijk om alle misvormingen die worden gezien in een spina bifida patiënt te beschrijven, ook als dit niet-neurologische afwijkingen betreft.

De trias van Currarino is een van de voorbeelden van een multidisciplinair raadsel, dat werd opgelost, maar er bestaan zeker andere syndromen die nog beschreven en genetisch geëvalueerd moeten worden. 


\section{References}

1. Adzick NS, Sutton LN, Crombleholme TM, Flake AW. Successful fetal surgery for spina bifida. Lancet 1998;352:1675-1676.

2. Altozano F. Un caso de sinus dermal congenito. Acta Pediatr Esp 1954;12:467-474.

3. Amador LV, Hankinson J, Bigler JA. Congenital spinal dermal sinuses. J Pediatr 1955;47: 300-310.

4. Brunelli G, Brunelli F. Experimental foetal microsurgery as related to myelomeningocele. Microsurgery 1984;5:24-29.

5. Bruner JP, Richards WO, Tulipan NB, Arney TL. Endoscopic coverage of fetal myelomeningocele in utero. Am J Obstet Gynecol 1999;180:153-158.

6. Cardell BS, Laurance B. Congenital dermal sinus associated with meningitis; report of a fatal case. Br Med J 1951;2:1558-1561.

7. Casani Martinez C, Otero Reigada MC, Perez Tamarit A, Alvarez Garijo JA, Asensi Botet F. [Lumbosacral dermal sinus as entry point of purulent meningitis. Report of 3 cases]. An Esp Pediatr 1991;34:68-70.

8. Currarino G, Coln D, Votteler T. Triad of anorectal, sacral, and presacral anomalies. AJR Am J Roentgenol 1981;137:395-398.

9. Dubreuilh MW. Abces Intra-medullaire consecutif a une tumeur dermoide congenitale. Bull Soc Anat Physiol Norm Pathol 1887;8:13-26.

10. Fuchs E. Beauty is skin deep: the fascinating biology of the epidermis and its appendages. Harvey Lect 1998;94:47-77.

11. Fuchs E. Scratching the surface of skin development. Nature 2007;445:834-842.

12. Fuchs E, Raghavan S. Getting under the skin of epidermal morphogenesis. Nat Rev Genet 2002;3:199-209.

13. Gupta DK, Shastank RR, Mahapatra AK. An unusual presentation of lumbosacral dermal sinus with CSF leak and meningitis. A case report and review of the literature. Pediatr Neurosurg 2005;41:98-101.

14. Heffez DS, Aryanpur J, Hutchins GM, Freeman JM. The paralysis associated with myelomeningocele: clinical and experimental data implicating a preventable spinal cord injury. Neurosurgery 1990;26:987-992.

15. Heffez DS, Aryanpur J, Rotellini NA, Hutchins GM, Freeman JM. Intrauterine repair of experimental surgically created dysraphism. Neurosurgery 1993;32:1005-1010.

16. Heij HA, Moorman-Voestermans CG, Vos A, Kneepkens CM. Triad of anorectal stenosis, sacral anomaly and presacral mass: a remediable cause of severe constipation. $\mathrm{Br} \mathrm{J}$ Surg 1990;77:102-104.

17. Herman JM, McLone DG, Storrs BB, Dauser RC. Analysis of 153 patients with myelomeningocele or spinal lipoma reoperated upon for a tethered cord. Presentation, management and outcome. Pediatr Neurosurg 1993;19:243-249.

18. Holzbeierlein J, Pope JI, Adams MC, Bruner J, Tulipan N, Brock JW, $3^{\text {rd }}$. The urodynamic profile of myelodysplasia in childhood with spinal closure during gestation. J Urol 2000; 164:1336-1339.

19. Kochling J, Karbasiyan M, Reis A. Spectrum of mutations and genotype-phenotype analysis in Currarino syndrome. Eur J Hum Genet 2001;9:599-605.

20. Kochling J, Pistor G, Marzhauser Brands S, Nasir R, Lanksch WR. The Currarino syndrome-hereditary transmitted syndrome of anorectal, sacral and presacral anomalies. Case report and review of the literature. Eur J Pediatr Surg 1996;6:114-119.

21. Lee SC, Chun YS, Jung SE, Park KW, Kim WK. Currarino triad: anorectal malformation, sacral bony abnormality, and presacral mass--a review of 11 cases. J Pediatr Surg 1997;32: 58-61.

22. Lynch SA, Bond PM, Copp AJ, Kirwan WO, Nour S, Balling R, Mariman E, Burn J, Strachan $T$. A gene for autosomal dominant sacral agenesis maps to the holoprosencephaly region at 7q36. Nat Genet 1995;11:93-95. 
23. Martinez-Lage JF, Perez-Espejo MA, Tortosa JG, Ros de San Pedro J, Ruiz-Espejo AM. Hydrocephalus in intraspinal dermoids and dermal sinuses: the spectrum of an uncommon association in children. Childs Nerv Syst 2006;22:698-703.

24. McLone DG, Dias MS, Goossens W, Knepper PA. Pathological changes in exposed neural tissue of fetal delayed splotch (Spd) mice. Childs Nerv Syst 1997;13:1-7.

25. Meuli M, Meuli-Simmen C, Hutchins GM, Yingling CD, Hoffman KM, Harrison MR, Adzick NS. In utero surgery rescues neurological function at birth in sheep with spina bifida. Nat Med 1995; 1:342-347.

26. Michejda M. Intrauterine treatment of spina bifida: primate model. Z Kinderchir 1984;39: 259-261.

27. Pang D. Split cord malformation: Part II: Clinical syndrome. Neurosurgery 1992;31:481-500.

28. Pang D, Dias MS, Ahab-Barmada M. Split cord malformation: Part I: A unified theory of embryogenesis for double spinal cord malformations. Neurosurgery 1992;31:451-480.

29. Pansey BK, Verma A, Sood PK, Chabra SC, Pansey M. Dermoid tumours occurring at the site of previous meningocele repair. Clin Neurol Neurosurg 1988;90:137-140.

30. Ripley W, Thompson DC. Pilonidal sinus as a route of infection in case of staphylococcus meningitis. Amer J Dis Child 1928;36:785-788.

31. Ross AJ, Ruiz-Perez V, Wang Y, Hagan DM, Scherer S, Lynch SA, Lindsay S, Custard E, Belloni E, Wilson DI, Wadey R, Goodman F, Orstavik KH, Monclair T, Robson S, Reardon W, Burn J, Scambler P, Strachan T. A homeobox gene, HLXB9, is the major locus for dominantly inherited sacral agenesis. Nat Genet 1998;20:358-361.

32. Salmon Hillbertz NH, Isaksson M, Karlsson EK, Hellmén E, Pielberg GR, Savolainen P, Wade $\mathrm{CM}$, von Euler H, Gustafson U, Hedhammar A, Nilsson M, Lindblad-Toh K, Andersson L, Andersson G. Duplication of FGF3, FGF4, FGF19 and ORAOV1 causes hair ridge and predisposition to dermoid sinus in Ridgeback dogs. Nat Genet 2007;39:1318-1320.

33. Scott RM, Wolpert SM, Bartoshesky LE, Zimbler S, Klauber GT. Dermoid tumors occurring at the site of previous myelomeningocele repair. J Neurosurg 1986;65:779-783.

34. Selcuki M, Manning S, Bernfield M. The curly tail mouse model of human neural tube defects demonstrates normal spinal cord differentiation at the level of the meningomyelocele: implications for fetal surgery. Childs Nerv Syst 2001;17:19-23.

35. Shenkin HA, Hunt AD, Horn RC. Sacrococcygeal sinus (pilonidal sinus) in direct continuity with the central canal of the spinal cord. Surg Gynecol Obstet 1944;79:655-659.

36. Storrs BB. Are dermoid and epidermoid tumors preventable complications of myelomeningocele repair? Pediatr Neurosurg 1994;20:160-162.

37. Sutton LN, Adzick NS, Johnson MP. Fetal surgery for myelomeningocele. Childs Nerv Syst 2003;19:587-591.

38. Tubbs RS, Chambers MR, Smyth MD, Bartolucci AA, Bruner JP, Tulipan N, Oakes WJ. Late gestational intrauterine myelomeningocele repair does not improve lower extremity function. Pediatr Neurosurg 2003;38:128-132.

39. Tulipan N. Intrauterine myelomeningocele repair. Clin Perinatol 2003;30:521-530.

40. Tulipan N, Sutton LN, Bruner JP, Cohen BM, Johnson M, Adzick NS. The effect of intrauterine myelomeningocele repair on the incidence of shunt-dependent hydrocephalus. Pediatr Neurosurg 2003;38:27-33.

41. van Aalst J, Beuls EA, Cornips EM, Vanormelingen L, Vandersteen M, Weber JW, Vles JS. Anatomy and surgery of the infected dermal sinus of the lower spine. Childs Nerv Syst 2006;22:1307-1315.

42. Weprin BE, Oakes WJ. Coccygeal pits. Pediatrics 2000;105:E69.

43. Yates VD, Wilroy RS, Whitington GL, Simmons JC. Anterior sacral defects: an autosomal dominantly inherited condition. J Pediatr 1983;102:239-242. 
Dankwoord 


\section{Dankwoord}

Op het moment dat u dit leest, is dit proefschrift klaar.

Het feit dat het ook werkelijk zover kwam is te danken aan Professor Beuls, Professor Vles en, hoewel het vanzelfsprekend ongepast is om te vermelden, ondergetekende.

Ooit begon ik met het schrijven van mijn eerste artikel, daarbij op alle vlakken voortdurend bijgestuurd door Professor Beuls. Dit casereport werd gepubliceerd en aangezien het zo leuk was om diep in een onderwerp te duiken en dat vervolgens op te schrijven, wilde ik dat graag nog eens doen en bleef ik bezig met artikelen te schrijven.

Een paar jaar en een paar artikelen later, adviseerden beide hoogleraren me om me verder te verdiepen in het onderwerp spina bifida en hier een proefschrift over te schrijven. Er moest dan echter nog wel wat werk verzet worden. Aldus werd besloten om dit te doen.

Prof. dr. Beuls, beste Emile. Voor deze ene keer ga ik je toch tutoyeren. Ik heb heel veel aan je te danken. Het vertrouwen dat je in mij en veel andere assistenten had en hebt was belangrijk en stimulerend. De vele uren die we samen hebben doorgebracht met het bestuderen van problemen of scans van bijzondere (spina)patiënten zijn voor mij zeer leerzaam geweest. Je manier van opereren is mooi en ik heb tijdens de operaties die we samen hebben gedaan ook ervaren wat begrippen als 'weefselgevoel' en 'chirurgische anatomie' eigenlijk betekenen. Daarnaast heb ik meer van je geleerd dan alleen neurochirurgie. Je manier van kijken naar een patiënt, met oprechte interesse en oog voor detail heb ik als bijzonder ervaren. Toen ik voor het eerst op een internationaal congres iets mocht presenteren zijn we samen met Micheline naar Argentinië gegaan. Wat een leuke reis was dat! lk weet niet meer wat ik het leukst vond aan dat congres, maar ergens in de top drie stond toch zeker dat jazzcafé waar ze die lekkere champagne schonken.

Prof. dr. Vles, beste Hans. Ik herinner me onze eerste ontmoeting (1997) nog goed. Je vertrouwde me direct je kamer, stoel en computer toe en legde me uit waar en hoe ik de relevante literatuur kon inzien. Vanaf die tijd ben je altijd zeer betrokken geweest bij alles wat ik op wetenschappelijk gebied (en vaak ook op andere gebieden) ondernam. Voorlopige versies van artikelen werden binnen no-time gelezen en van goed commentaar voorzien en tijdens het schrijven van mijn proefschrift functioneerde je als een rots in de soms woelige branding. Je bent een belangrijke factor en drijvende kracht geweest in de totstandkoming van dit proefschrift. Ik heb diepe bewondering voor je klinische, wetenschappelijke en ook kritische blik en hoop dat onze kliniek daar nog lang van 
mag genieten. Daarnaast heb ik ook veel geleerd van de manier waarop je naar werk en leven kijkt. Hartelijk dank daarvoor.

Prof. dr. van Overbeeke, beste Koo, als nieuw afdelingshoofd heb je me onvoorwaardelijk gesteund in mijn voornemen om dit proefschrift af te ronden. Hiervoor dank. Daarnaast dank ik je voor je rol als voorzitter van de beoordelingscommissie van mijn proefschrift. 'In gesprek komen met de tumor' is trouwens nu al een begrip in Maastricht.

Dr. van Straaten, beste Henny, zonder jouw kennis en ervaring had dit proefschrift er anders uitgezien. Jouw lab, waarin je me met open armen hebt ontvangen, was al jaren gespecialiseerd in dit onderwerp en kon ook nog eens een relevant diermodel ter beschikking stellen. De manier waarop dit ging was leuk en stimulerend, net als je vaak duidelijke mening over allerlei embryologische zaken.

Beste Johan Hekking en Paul van Dijk, jullie hebben me op een plezierige manier wegwijs gemaakt in het anatomie/embryologielab en vaak uit de brand geholpen. Hartelijk dank daarvoor. Ik had trouwens nooit gedacht dat er af en toe babypapegaaien naast de snijzaal wonen!

Drs. Cornips, beste Erwin, niet alleen heb jij meerdere kinderen die beschreven zijn in dit proefschrift geopereerd, maar ook was je altijd bereid om een van onze artikelen nog eens bijzonder grondig te reviseren. Bedankt voor al deze uren revisiewerk! Daarnaast heb ik veel van je geleerd op klinisch en operatief vlak.

Drs. Boselie, beste Toon, samen hebben we vele uren doorgebracht tussen de kippeneieren. Naast het feit dat er hard gewerkt werd, hebben we ook ontzettend veel lol gehad. Voor je onmisbare hulp (en de lol) tijdens dat onderzoek wil ik je graag bedanken.

Drs. Hoekstra, beste Franka, bedankt voor al het werk dat je hebt gedaan betreffende de intraspinale inclusietumoren. Zonder jouw hulp was dat artikel er misschien wel nooit gekomen!

Ook tot nu toe niet genoemde collega's die mee hebben gewerkt aan het totstandkomen van een van de artikelen dank ik voor hun bijdrage aan dit proefschrift.

Spina bifida team Maastricht, naast eerder genoemde mensen en alle leden van het spinateam wil ik vooral Biene Weber (kinderneuroloog) en Dan 
Soudant (coördinator spinateam) bedanken. Ik heb veel profijt gehad van de goede database en correspondentie die binnen het spinateam aanwezig is.

Leden van de beoordelingscommissie: Prof. dr. F. van Calenbergh (Universitair Ziekenhuis Leuven), Dr. C.E.M. de Die-Smulders, Prof. dr. L.W. van Rhijn en Dr. D.A. Sival (UMC Groningen). Bedankt voor het kritisch lezen en beoordelen van mijn proefschriftconcept.

Dr. A. Petrasch, beste Alexander, ik dank je voor alles wat je me hebt geleerd, want dat heeft wezenlijk bijgedragen aan wie ik ben. Naast het feit dat je me met grote betrokkenheid hebt begeleid tot aan mijn examen als Uitvoerend Musicus, heb ik meer van je geleerd dan alleen muziekmaken. De vele uren discussie over politiek, filosofie en menselijk gedrag zal ik niet vergeten.

Neurochirurgen, kinderneurologen, neuroradiologen, kinder-anaesthesisten, kinderartsen, kinder-intensivisten, assistenten, secretariaten, poli en verpleging Maastricht en Heerlen, het is niet te doen om iedereen afzonderlijk te bedanken en dat zou ook zeker onvolledig worden. Bedankt voor de goede samenwerking, het medeleven, de opleiding en de vriendschap tijdens het schrijven van dit boekje.

Beste Ber Eggen, bedankt voor je mooie illustraties. Maar weinig mensen in Maastricht weten dat hun voormalig Prins Carnaval meewerkt aan neurochirurgische dissertaties.

Beste Tiny Wouters, onmisbare hulp. Ontzettend bedankt voor je hulp met de lay-out en het drukklaar maken van dit boekje. Ik zou niet weten hoe ik dat op een andere manier voor elkaar had gekregen!

Mijn paranimfen René de Bruijn en Ward Vanagt, het is een prettig gevoel dat jullie me tijdens de verdediging van dit proefschrift zullen bijstaan. René, mijn privé-psychiater, wat hebben wij ontzettend veel besproken de afgelopen 10 jaar! Gelukkig hebben we daar ook ontzettend veel bij gegeten en gedronken. Ward, bedankt voor het feit dat je er werkelijk 'altijd bent'. Ook bedankt trouwens voor de cadeautjes (zoals 'echt' heilig water uit Jordanië, of een hangstoel uit Costa Rica) die je voor ons koopt als je er niet bent! Gelukkig is het proefschrift nu af, want daar heb je genoeg over aangehoord. Biertje?

Lieve $\mathrm{Pa}$ en $\mathrm{Ma}$, bedankt voor het warme nest. Ook later, vanuit Zeist, Birkerød, Brussel of Brummen was dat altijd aanwezig. Ik hoop dat we wat vaker naar jullie, de kippen en de schapen kunnen komen kijken nu dit werkje af is. 
Lieve Kim en Wouter, in dit dankwoord zijn al meerdere superlatieven gebruikt, maar die kunnen toch niet omschrijven wat ik aan jullie te zeggen heb. Dat doe ik dus ook niet in dit dankwoord. Toch heb ik wel iets aan jullie te zeggen. Lieve Kim, ook jij hebt een belangrijke rol in de totstandkoming van dit proefschrift gehad, maar dat is niets vergeleken bij de rol die je speelt in mijn leven! Bedankt voor je warmte en liefde.

Lief Woutertje, misschien blader je dit boekje ooit nog eens door als je groot bent. Probeer dan vooral niet alles te begrijpen. Er staan trouwens best mooie plaatjes in! 



\section{List of publications}





\section{List of publications}

1. van Aalst J, Beuls EA, van Nie FA, Vles JS, Cornips EM. Acute distortion of the anatomy of the third ventricle during third ventriculostomy. Report of four cases.J Neurosurg 2002;96:597-599.

2. van Aalst J, Beuls EA, Luijckx GJ. Neuropsychological and psychiatric complications in endoscopic third ventriculostomy. J Neurol Neurosurg Psychiatry 2002;73:460.

3. Beuls EA, Vanormelingen L, van Aalst $J$, Vandersteen $M$, Adriaensen $P$, Cornips EM, Vles JS, Gelan J. In vitro high-field magnetic resonance imaging-documented anatomy of a fetal myelomeningocele at 20 weeks' gestation. A contribution to the rationale of intrauterine surgical repair of spina bifida. J Neurosurg 2003;98(2 Suppl):210-214.

4. Beuls EA, Vanormelingen L, van Aalst J, Vandersteen M, Adriaensen $P$, Cornips EM, Vles JS, Temel Y, Gelan J. The Arnold-Chiari type II malformation at midgestation. Pediatr Neurosurg 2003;39:149-158.

5. van Aalst J, Beuls EA, Vles JS, Cornips EM, van Straaten HW. The intermediate type split cord malformation: hypothesis and case report. Childs Nerv Syst 2005;21:1020-1024.

6. Emans PJ, van Aalst J, van Heurn EL, Marcelis C, Kootstra G, Beets-Tan RG, Vles JS, Beuls EA. The Currarino triad: neurosurgical considerations. Neurosurgery 2006;58:924-929.

7. van Aalst J, Beuls EA, Cornips EM, Vanormelingen L, Vandersteen M, Weber JW, Vles JS. Anatomy and surgery of the infected dermal sinus of the lower spine. Childs Nerv Syst 2006:22:1307-1315.

8. Cornips EM, Beuls EA, Geskes G, Janssens M, van Aalst J, Hofman P. Preoperative localization of herniated thoracic discs using myelo-CT guided transpleural puncture: technical note. Childs Nerv Syst 2007;23:21-26.

9. van Aalst J, Beuls EAM, Cornips EMJ, van Straaten HWM, Boselie AFM, Rijkers K, Weber JW, Vles JSH. The Spinal dermal sinus-like stalk. Childs Nerv Syst 2009:25:191-197.

10. Rijkers K, van Aalst J, Kurt E, Daemen MA, Beuls EA, Spincemaille GH. Spinal cord stimulation in Type I Complex Regional Pain Syndrome; instant effect on two rare severe cutaneous manifestations. J Neurosurg 2009;110:274-278.

11. van Aalst J, Boselie AFM, Beuls EAM, Vles JSH, van Straaten HWM. Spinal congenital dermal sinus in a chick embryo model. Laboratory investigation. J Neurosurg Pediatr 2009;3:24-28.

12. van Aalst J, Hoekstra F, Beuls EA, Cornips EM, Weber JW, Sival DA, Creytens DH, Vles JS. Intraspinal dermoid and epidermoid tumors: report of 18 cases and reappraisal of the literature. Pediatr Neurosurg 2009;45: 281-290. 

Curriculum Vitae 



\section{Curriculum Vitae}

Jasper van Aalst was born on May 2, 1975 in Den Haag, The Netherlands. From 1991 to 1994 he studied cello at the Utrecht school of the Arts. After finishing his secondary education at the Vrije School Zeist in 1994, he started his medical training at Maastricht University. In 2001 he obtained his medical degree. In 1995 he restarted his musical education, and studied cello with Dr. Alexander Petrasch at the Maastricht Academy of Music, where he obtained his Bachelor of Music degree in 1999.

In 2001 he started as a resident at the neurosurgical department of the Maastricht University Hospital, where in 2003 he commenced his neurosurgical training with Prof. dr. E.A.M. Beuls and Prof. dr. J.J. van Overbeeke. During his residency he started the research described in this thesis under supervision of Prof. dr. E.A.M. Beuls and Prof. dr. J.S.H. Vles.

In February 2010, he will complete his neurosurgical training.

Jasper van Aalst werd op 2 Mei 1975 geboren in Den Haag. Van 1991 tot 1994 studeerde hij cello aan het Conservatorium van Utrecht. Na in 1994 aan de Vrije School te Zeist het atheneum examen te hebben behaald, studeerde hij vanaf 1994 Geneeskunde aan de Universiteit Maastricht. Daar werd in 2001 het artsexamen afgelegd.

Vanaf 1995 studeerde hij cello aan het Conservatorium van Maastricht. In 1999 studeerde hij bij Dr. Alexander Petrasch af als Uitvoerend Musicus.

Vanaf 2001 was hij werkzaam als arts-assistent neurochirurgie in het academisch ziekenhuis Maastricht, alwaar hij vanaf 2003 onder leiding van Prof. dr. E.A.M. Beuls, en vanaf 2008 onder leiding van Prof. dr. J.J. van Overbeeke, in opleiding is tot neurochirurg.

Tijdens deze opleiding werd, onder begeleiding van Prof. dr. E.A.M. Beuls en Prof. dr. J.S.H. Vles het proefschrift geschreven dat nu voor u ligt. In Februari 2010 zal Jasper de opleiding tot neurochirurg afronden. 

Colour figures 


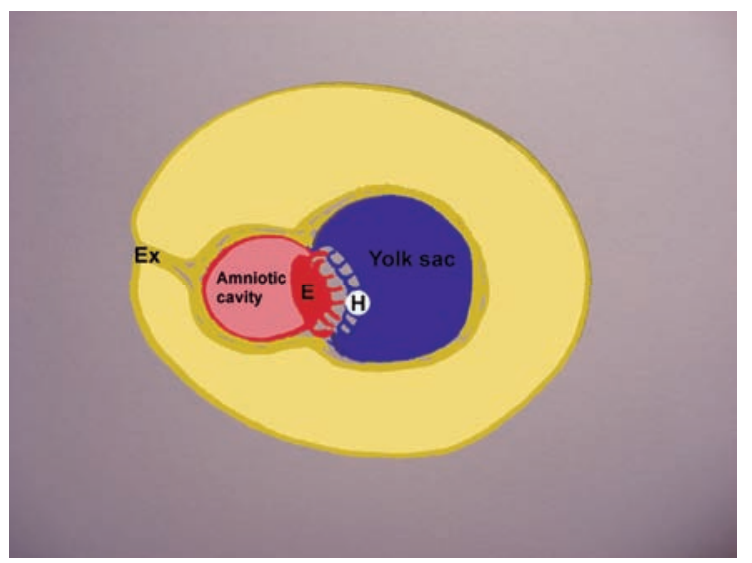

Figure 1.1 Ex = Extraembryonic mesoderm, E = Epiblast, $\mathrm{H}=$ Hypoblast.

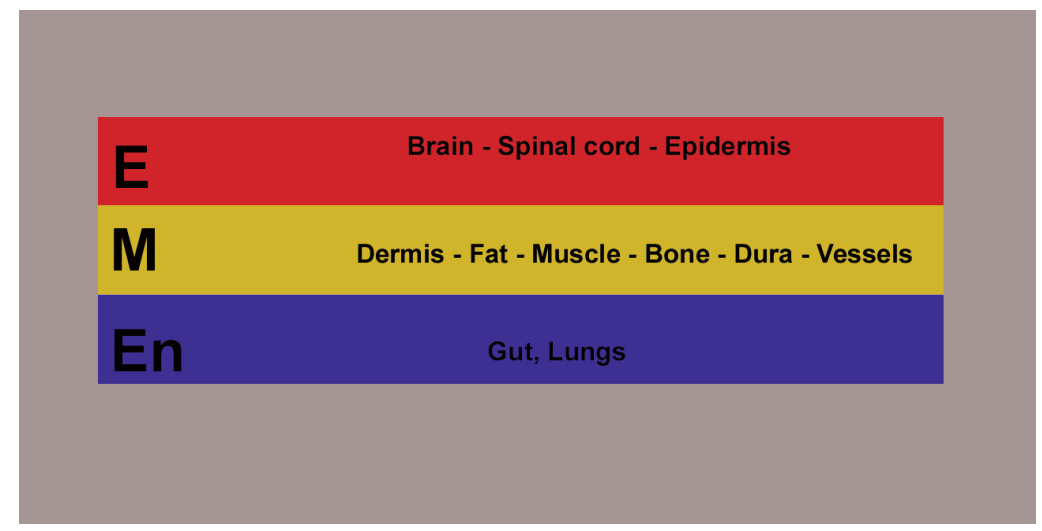

Figure 1.2 $E=$ ectoderm, $M=$ mesoderm, $E n=$ Endoderm. 


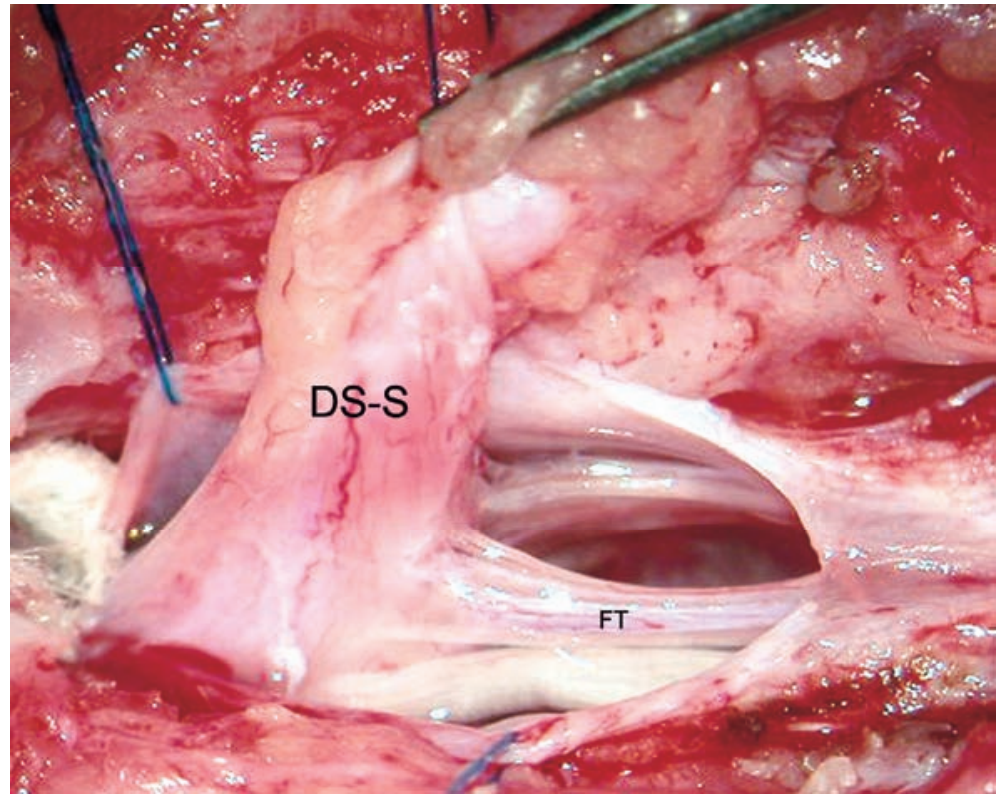

Figure 4.2 Intraoperative picture (case 1), showing the dermal sinus-like stalk (DS-S) being attached to the conus medullaris. FT = filum terminale.

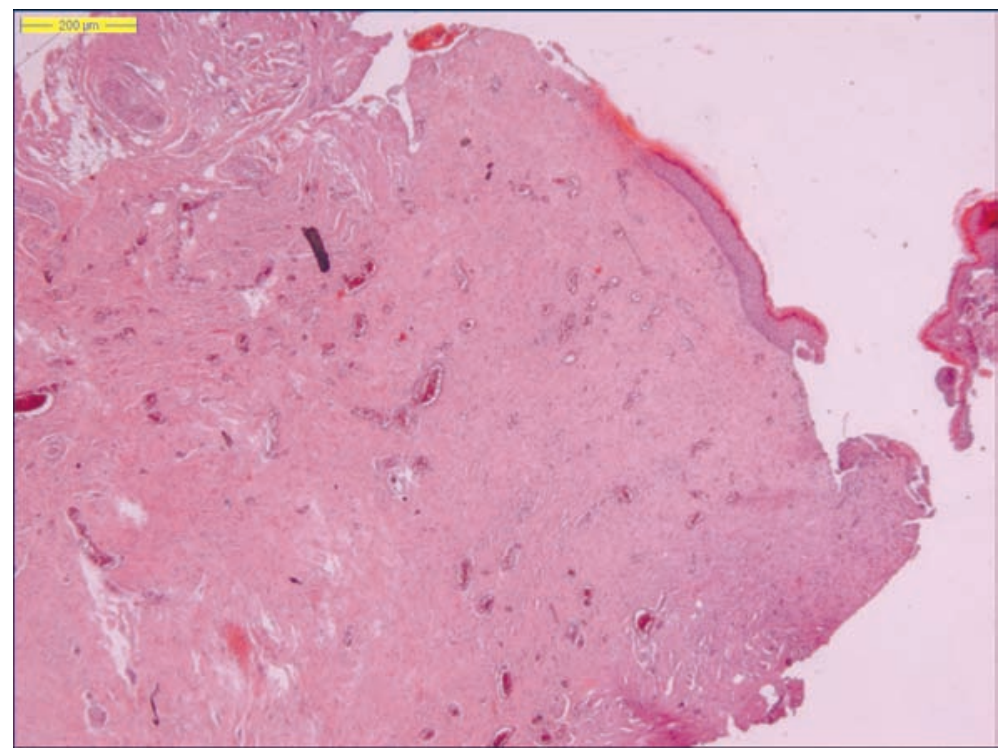

Figure 4.3 Photomicrograph of the resected stalk in case 1. Most of it is composed of connective tissue. 

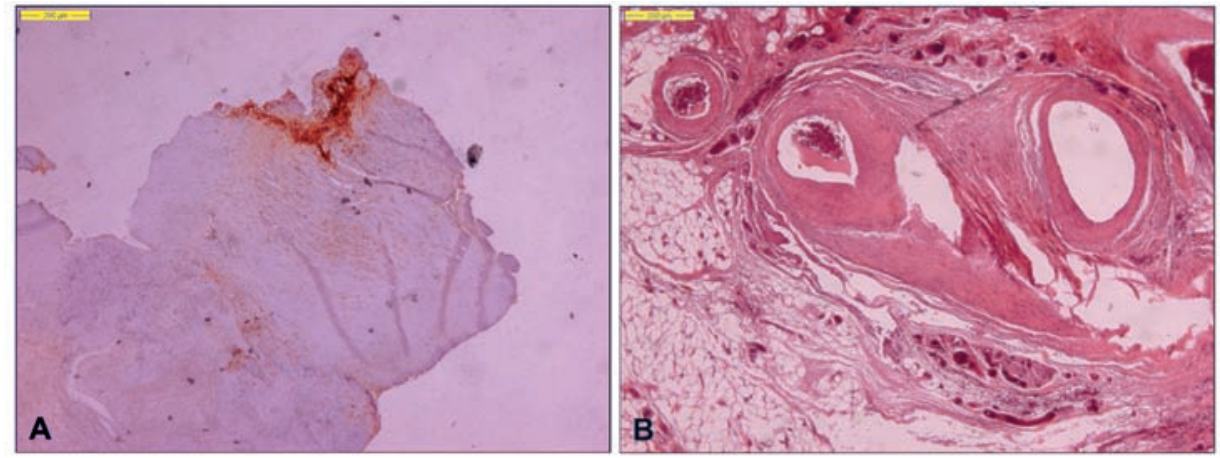

Figure 4.5 Photomicrographs of the resected stalk in case 2. Some areas are EMA positive (A). Note the large vessels and a considerable amount of fat (B).

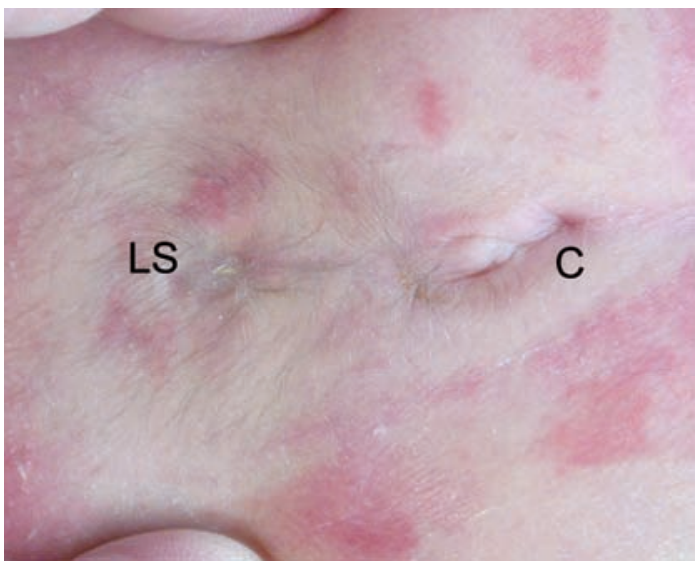

Figure 4.6 Preoperative picture of dimples in case 3. C = coccygeal, LS = lumbosacral. 


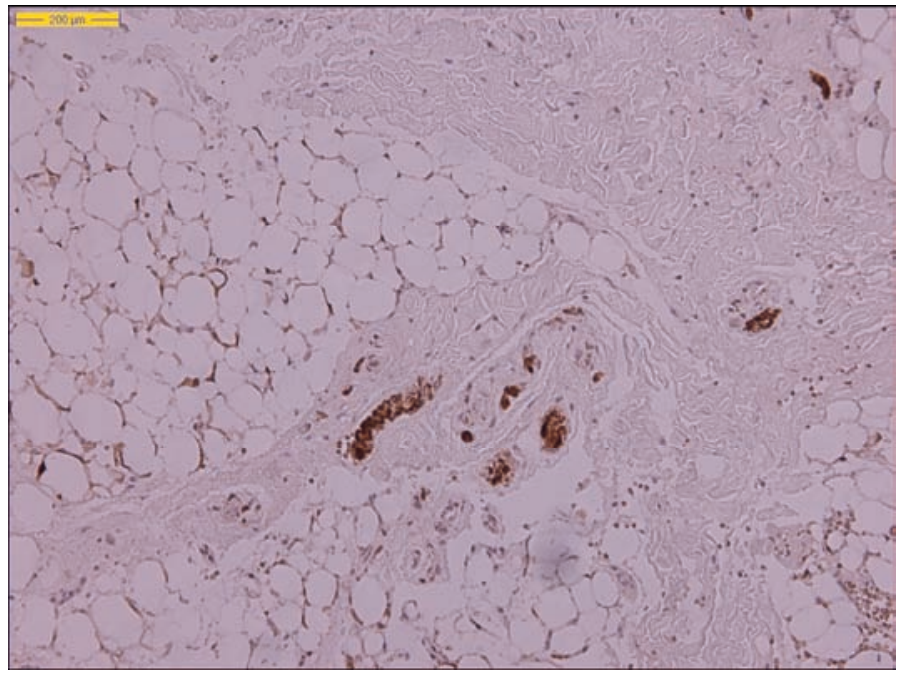

Figure 4.7 Photomicrograph of the resected stalk in case 3. Note the large fat cells and the small parts, which are positively stained with S100. (H\&E, S100 staining).

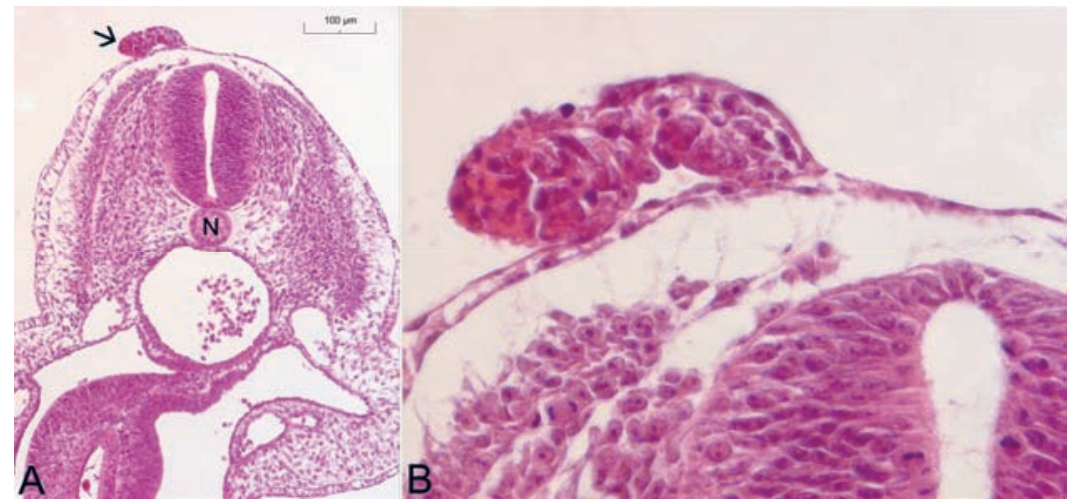

Figure 5.2 Photomicrographs. A: A tract of tissue (arrow) protrudes from the epidermis. $\mathrm{N}=$ notochord. $\mathrm{B}$ : Enlargement of Figure 5.2A. The pink tissue inside the protrusion has the appearance of amnion. H\&E staining, original magnification x 1250. 


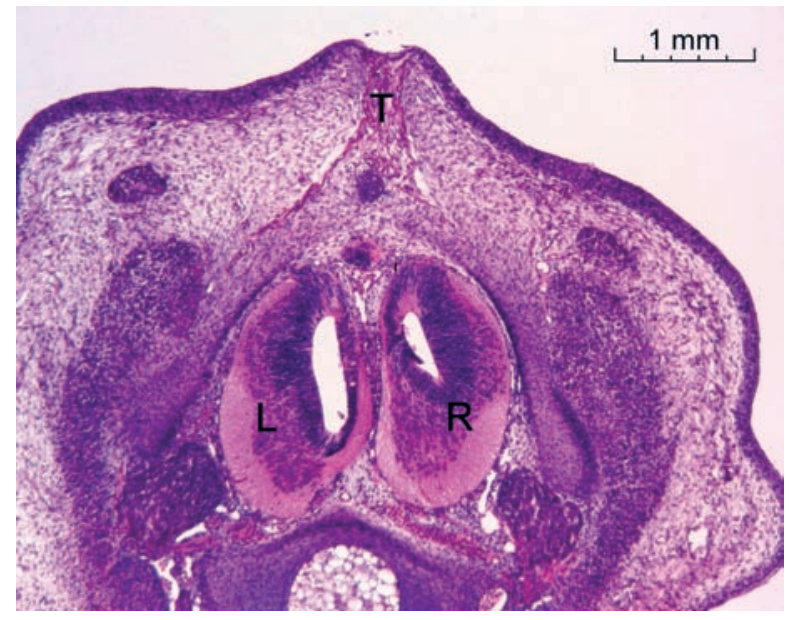

Figure 5.3 Photomicrograph. HE staining. Example of a split cord malformation as was seen in some of the embryos. $\mathrm{T}=$ tract, $\mathrm{L}$ and $\mathrm{R}=$ left and right hemicord.

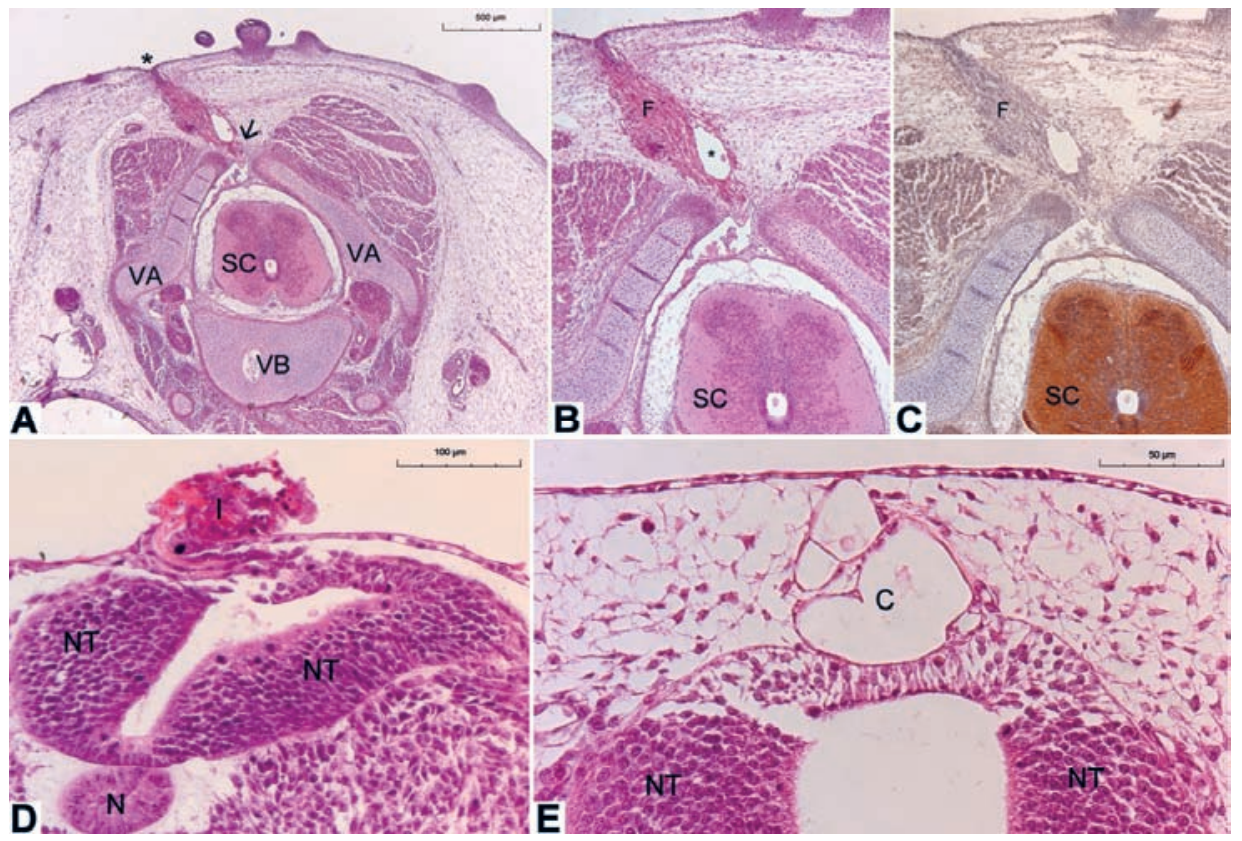

Figure 5.4 Photomicrographs. A: A section obtained in a 9-day embryo. An epidermal dimple (indicated by the asterisk) is present, attached to a tract, which continues through a closure defect (arrow) in the vertebral arch (VA). The vertebral body (VB) and spinal cord (SC) have a normal appearance. B. Enlargement of panel A. Note the vacuole (asterisk) within the fibrous tract $(\mathrm{F})$. C. Staining of the spinal cord (SC) but not of the fibrous tract. D. Three-day embryo; the implant (I) seems to infiltrate in the disturbed neural tube (NT). The notochord $(\mathrm{N})$ is seen underlying the neural tube. $\mathrm{E}$. Four-day embryo; cystic tract $(C)$, which makes contact with the neural tube. $\mathrm{H}$ \& $\mathrm{E}$ (A, B, D, and E); NCAM (C); original magnification $\times 85$ (B and C). 

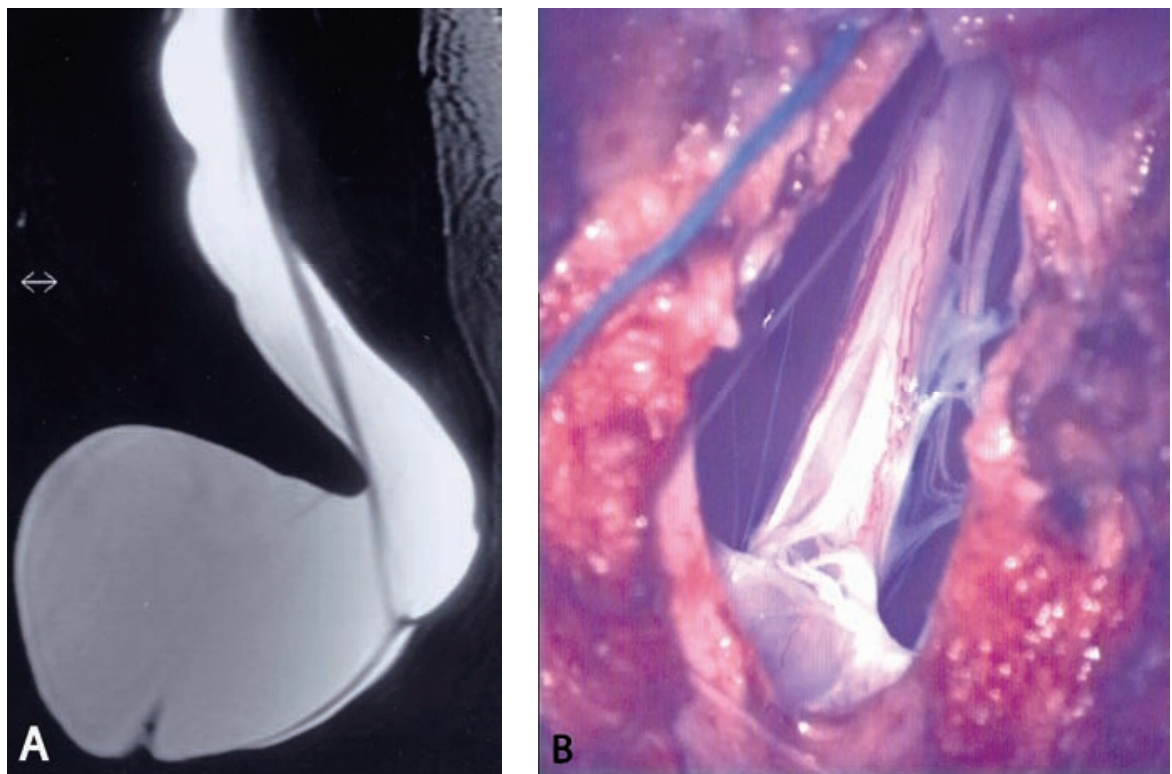

Figure 7.2 A. Preoperative MR image of AMMC. B. Intraoperative view of AMMC. Both panels show the complex anatomy at site of adherence of the lower spinal cord to the dura mater.

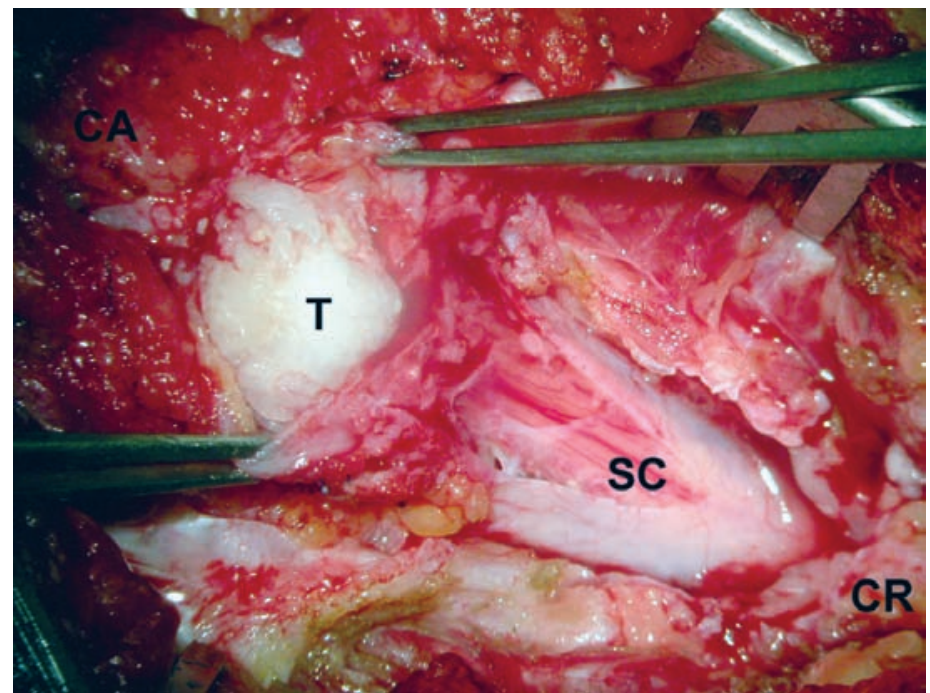

Figure 8.3 Intraoperative photograph of an intramedullary tumor at the level of the conus medullaris. In this case the radiological diagnosis was made of an epidermoid, whereas the surgeon reported a dermoid. Histopathological re-assessment of the tumor specimen revealed only desquamated cells. $\mathrm{CR}=$ cranial, $\mathrm{CA}=$ caudal, $\mathrm{SC}=$ spinal cord, $\mathrm{T}=$ tumor. 


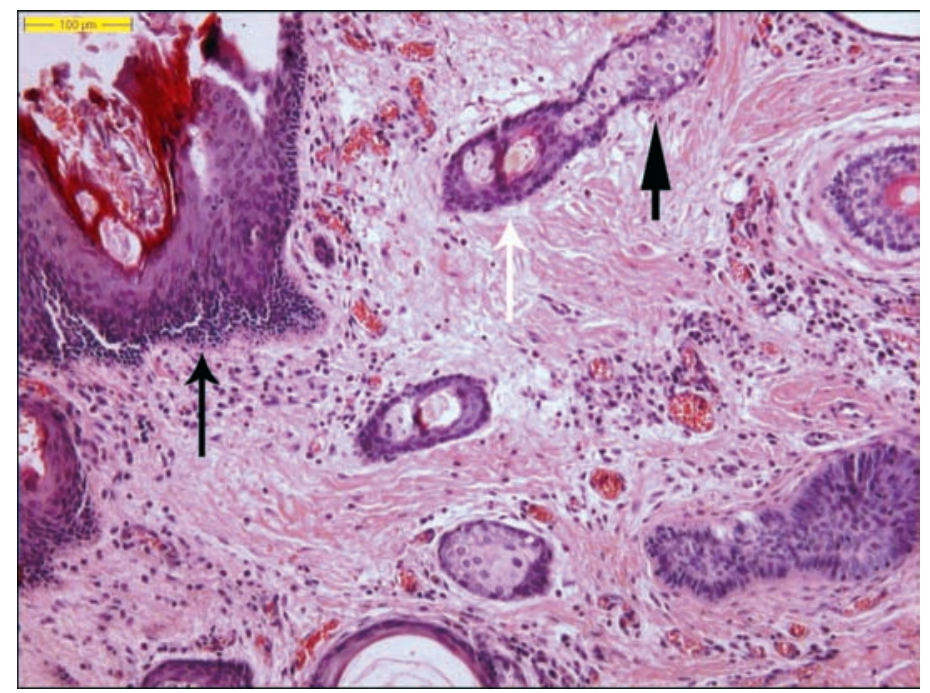

Figure 8.4 HE-staining. Photomicrograph of histopathology specimen showing a dermoid tumor. The wall of the tumor is lined by stratified squamous epithelium (black arrow). Hair follicles (white arrow) and sebaceous glands (arrow head) are present.

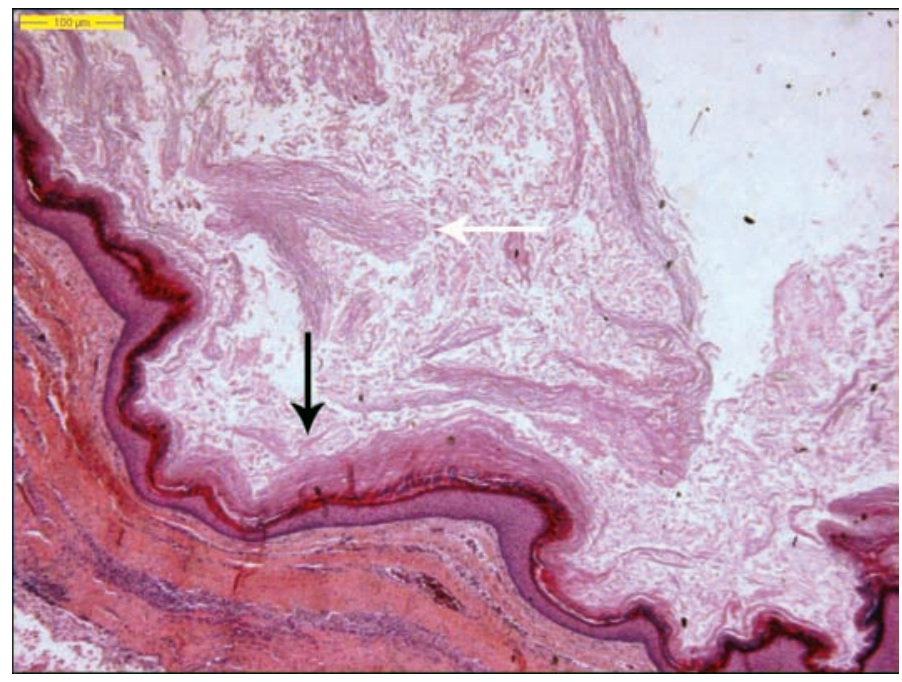

Figure 8.5 HE-staining. Photomicrograph of histopathology specimen showing an epidermoid tumor. The wall of the tumor is lined by stratified squamous epithelium (black arrow). The content of the tumor consists of laminated keratin and desquamated epithelial cells (white arrow). No epidermal adnexa are present. 